Supporting Information

\title{
a-Oxocarboxylic Acids as Three-Carbon Insertion Units for Palladium-Catalyzed Decarboxylative Cascade Synthesis of Diverse Fused Heteropolycycles
}

Liwei Zhou, Shujia Qiao, Fengru Zhou, Xinyu Xuchen, Guobo Deng, Yuan Yang,* and Yun Liang*

National \& Local Joint Engineering Laboratory for New Petro-chemical Materials and Fine Utilization of Resources, Key Laboratory of Chemical Biology and Traditional Chinese Medicine Research, Ministry of Education, Key Laboratory of the Assembly and Application of Organic Functional Molecules of Hunan Province, Hunan Normal University, Changsha, Hunan 410081, China.

E-mail: yuanyang@hunnu.edu.cn; yliang@hunnu.edu.cn. 


\section{Table of Contents}

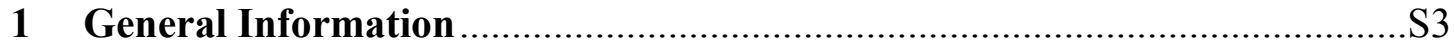

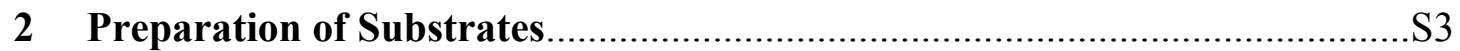

2.1 General Procedure for the Synthesis of Substrates 1a-1n ${ }^{[1,2]}$.............S3

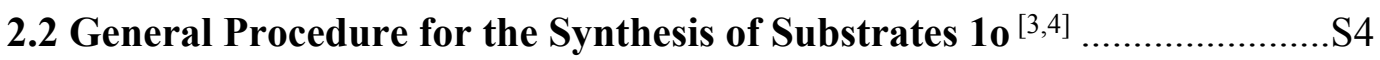

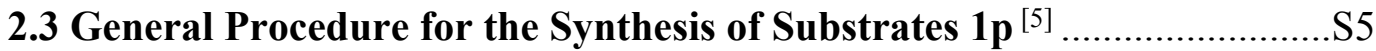

2.4 General Procedure for the Synthesis of Substrates $4 a-4 g^{[6,7,8]} \ldots \ldots \ldots \ldots \ldots \ldots . . .56$

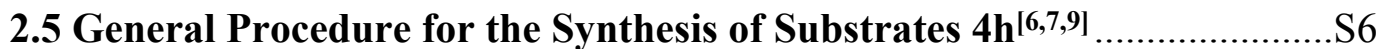

2.6 General Procedure for the Synthesis of Substrates $2 \mathrm{a}-2]^{[10,11]}$................S7

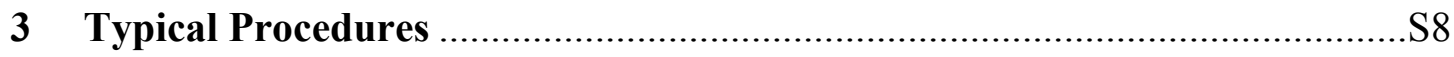

3.1 Typical Procedures for the synthesis of Isoquinolinedione-Fused

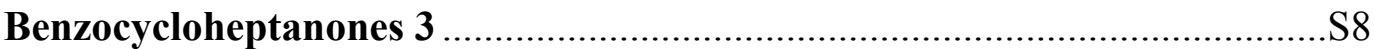

3.2 General Procedure for the Synthesis of

Indolo[2,1-a]isoquinolinone-Fused Benzocycloheptanones 5.......................S9

3.3 General Procedure for the Synthesis of 3aa from 1 mmol Scale of 1a S9



5 Crystal Culture Procedure of Product 3aa...................................................S12

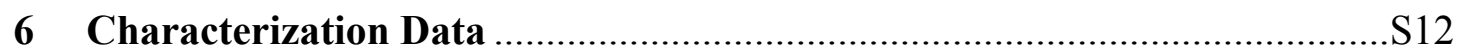

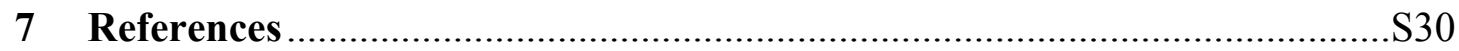

8 Scanned ${ }^{1} \mathrm{H}$ NMR and ${ }^{13} \mathrm{C}$ NMR Spectra of All Compounds .......................S31

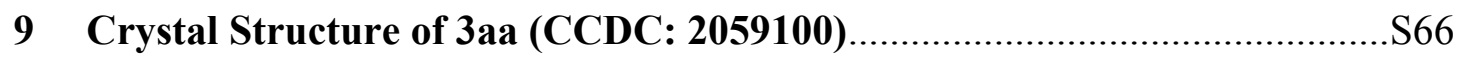




\section{General Information}

${ }^{1} \mathrm{H}-\mathrm{NMR}$ and ${ }^{13} \mathrm{C}-\mathrm{NMR}$ spectra were recorded at room temperature using a Bruker Avance-500 instruments or Avance-400 instruments $\left({ }^{1} \mathrm{H}\right.$ NMR at $500 \mathrm{MHz}$ and ${ }^{13} \mathrm{C} \mathrm{NMR}$ at $125 \mathrm{MHz}$ ), NMR spectra of all products were reported in ppm with reference to solvent signals $\left[{ }^{1} \mathrm{H} \mathrm{NMR}: \mathrm{CD}(\mathrm{H}) \mathrm{Cl}_{3}(7.26 \mathrm{ppm}),{ }^{13} \mathrm{C} \mathrm{NMR}: \mathrm{CD}(\mathrm{H}) \mathrm{Cl}_{3}\right.$ (77.00 ppm)]. Signal patterns are indicated as s, singlet; d, doublet; dd, doublets of doublet; $\mathrm{t}$, triplet, and m, multiplet. HPLC/Q-TOF-MS analysis was performed with an Agilent 1290 LC system coupled with a 6530Q-TOF/MS accurate-mass spectrometer (Agilent Technologies, USA). The mass spectrometry was performed in the positive electrospray ionization $(\mathrm{ESI}+)$ mode. Reactions were monitored by thin-layer chromatography Column chromatography (petroleum ether/ethyl acetate) was performed on silica gel (200-300 mesh). Analytical grade solvents and commercially available reagents were purchased from commercial sources and used directly without further purification unless otherwise stated.

\section{Preparation of Substrates}

\subsection{General Procedure for the Synthesis of Substrates 1a-1n ${ }^{[1,2]}$}


An oven dried reaction tube containing a PTFE-coated stir bar was charged with 2-iodobenzoic acid (5.0 mmol, 1.0 equiv, $1.24 \mathrm{~g}$ ), oxalyl chloride (6.0 mmol, 1.2 equiv, $0.5 \mathrm{~mL})$ in dichloromethane $(10 \mathrm{ml})$ and DMF (3 drops). The mixture was stirred by 2 hours at $0{ }^{\circ} \mathrm{C}$ and was warmed at room temperature. The solvent was evaporated and the crude was used directly in the next step. The 2-iodobenzoyl chloride (5.0 mmol, 1.0 equiv, $1.33 \mathrm{~g})$ was dissolved in dichloromethane $(10 \mathrm{ml})$. Phenylmethanamine (10.0 mmol, 2.0 equiv, $1.1 \mathrm{~mL}$ ) (in some cases is necessary to add 2.0 equiv more) and $\mathrm{Et}_{3} \mathrm{~N}(10.0 \mathrm{mmol}, 2.0$ equiv, $1.4 \mathrm{~mL})$ were added and the mixture was stirred at $0{ }^{\circ} \mathrm{C}$ until consumption of acylchloride. The corresponding amide was isolated after addition of $5 \mathrm{~mL}$ of saturated $\mathrm{Na}_{2} \mathrm{CO}_{3}$ solution and extraction 
with dichoromethane. It was used without any purification in the next step. Methacryloyl chloride $(5.0 \mathrm{mmol}, 1.0$ equiv, $0.59 \mathrm{~mL})$ in dichoromethane $(5 \mathrm{ml})$ was added on a mixture of 2-iodo- $N$-methylbenzamide (5.0 mmol, 1.0 equiv, $1.31 \mathrm{~g}$ ), triethylamine (10.0 mmol, 2.0 equiv, $1.4 \mathrm{~mL})$ and DMAP (0.25 mmol, 0.05 equiv, 39 $\mathrm{mg}$ ) in toluene $(5 \mathrm{ml})$. The mixture was stirred overnight at reflux in oil bath. The reaction was quenched with saturated aqueous $\mathrm{Na}_{2} \mathrm{CO}_{3}(5 \mathrm{~mL})$, then the mixture was extracted with dichloromethane $(3 \times 5 \mathrm{~mL})$. The combined organic layers were dried over $\mathrm{Na}_{2} \mathrm{SO}_{4}$, filtered, and concentrated in vacuo. The residue was purified by column chromatography on silica gel (eluent: petroleum ether/EtOAc $=10: 1$ ) affording the corresponding product 1a.

The procedures of $\mathbf{1 b}$ to $\mathbf{1 n}$ were similar to $\mathbf{1 a}$.

\subsection{General Procedure for the Synthesis of Substrates 1o ${ }^{[3,4]}$}
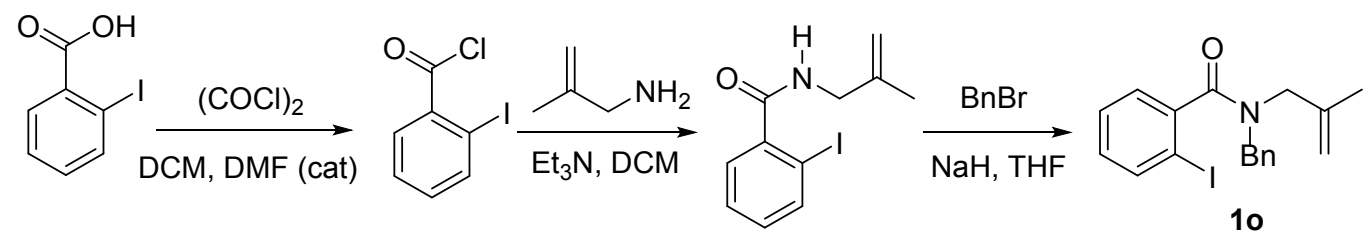

An oven dried reaction tube containing a PTFE-coated stir bar was charged with 2-iodobenzoic acid (5.0 mmol, 1.0 equiv, $1.24 \mathrm{~g}$ ), oxalyl chloride (6.0 mmol, 1.2 equiv, $0.5 \mathrm{~mL})$ in dichloromethane $(10 \mathrm{ml})$ and DMF (3 drops). The mixture was stirred by 2 hours at $0{ }^{\circ} \mathrm{C}$ and was warmed at room temperature. The solvent was evaporated and the crude was used directly in the next step. The 2-iodobenzoyl chloride (5.0 mmol, 1.0 equiv, $1.33 \mathrm{~g}$ ) was dissolved in dichloromethane $(10 \mathrm{ml})$. 2-Methylprop-2-en-1-amine (10.0 mmol, 2.0 equiv, $0.91 \mathrm{~mL}$ ) (in some cases is necessary to add 2.0 equiv more) and $\mathrm{Et}_{3} \mathrm{~N}$ (10.0 mmol, 2.0 equiv, $1.4 \mathrm{~mL}$ ) were added and the mixture was stirred at $0{ }^{\circ} \mathrm{C}$ until consumption of acyl chloride. The corresponding amide was isolated after addition of $5 \mathrm{~mL}$ of saturated $\mathrm{Na}_{2} \mathrm{CO}_{3}$ solution and extraction with dichoromethane. It was used without any purification in the next step.

$\mathrm{NaH}(2.0 \mathrm{mmol}, 2.0$ equiv, $80 \mathrm{mg}, 60 \%$ in mineral oil) was added to a solution of the above product in $\mathrm{THF}(5 \mathrm{~mL})$ at $0{ }^{\circ} \mathrm{C}$ in portions. After stirring for $20 \mathrm{~min}$ at $0{ }^{\circ} \mathrm{C}$, 
(bromomethyl)benzene ( $3.0 \mathrm{mmol}, 3.0$ equiv, $0.4 \mathrm{~mL}$ ) was added dropwise and the reaction mixture was allowed to room temperature and stirred for another $1 \mathrm{~h}$. After completion of the reaction (monitored by TLC), the residue was quenched with water and extracted into ethyl acetate. The organic layer was dried over anhydrous sodium sulfate and the solution was evaporated to dryness. The crude product was purified by column chromatography (eluent: petroleum ether/EtOAc $=10: 1$ ) to provide the desired product 10.

\subsection{General Procedure for the Synthesis of Substrates $1 p^{[5]}$}<smiles>C=C(C)C(=O)N(Cc1ccccc1I)Cc1cccc(I)c1CNc1cccc(I)c1C</smiles>

The 1-(bromomethyl)-2-iodobenzene $(5.0 \mathrm{mmol}, 1.0$ equiv, $1.48 \mathrm{~g})$ was dissolved in dichloromethane $(10 \mathrm{ml})$. Phenylmethanamine $(10.0 \mathrm{mmol}, 2.0$ equiv, 1.1 $\mathrm{mL}$ ) (in some cases is necessary to add 2.0 equiv more) and $\mathrm{K}_{2} \mathrm{CO}_{3}$ (10.0 mmol, 2.0 equiv, $1.38 \mathrm{~g}$ ) were added and the mixture was stirred at $0{ }^{\circ} \mathrm{C}$ overnight. The reaction was quenched with saturated aqueous $\mathrm{Na}_{2} \mathrm{CO}_{3}(5 \mathrm{~mL})$, then the mixture was extracted with dichloromethane $(3 \times 5 \mathrm{~mL})$. The combined organic layers were dried over $\mathrm{Na}_{2} \mathrm{SO}_{4}$, filtered, and concentrated in vacuo. The residue was purified by column chromatography on silica gel (eluent: petroleum ether/EtOAc $=30: 1$ ).

Methacryloyl chloride $(5.0 \mathrm{mmol}, 2.0$ equiv, $0.6 \mathrm{~mL})$ in dichoromethane $(5 \mathrm{ml})$ was added on a mixture of amide $(2.5 \mathrm{mmol}, 1.0$ equiv, $620 \mathrm{mg})$, triethylamine (10.0 mmol, 2.0 equiv, $1.4 \mathrm{~mL})$ and DMAP $(0.25 \mathrm{mmol}, 0.05$ equiv, $15 \mathrm{mg})$ in dichoromethane $(10 \mathrm{ml})$. The mixture was stirred overnight at reflux in oil bath. The reaction was quenched with saturated aqueous $\mathrm{Na}_{2} \mathrm{CO}_{3}(5 \mathrm{~mL})$, then the mixture was extracted with dichloromethane $(3 \times 5 \mathrm{~mL})$. The combined organic layers were dried over $\mathrm{Na}_{2} \mathrm{SO}_{4}$, filtered, and concentrated in vacuo. The residue was purified by column chromatography on silica gel (eluent: petroleum ether/EtOAc $=10: 1$ ) affording the corresponding product $\mathbf{1 p}$. 


\subsection{General Procedure for the Synthesis of Substrates $4 a_{-4}{ }^{[6,7,8]}$}<smiles>C=C(C)C(=O)n1c(-c2ccccc2I)cc2ccccc21</smiles>

A mixture of 2-iodoacetophenone (5.0 mmol, 1.0 equiv, $1.23 \mathrm{~g})$, phenylhydrazine (6.0 mmol, 1.2 equiv, $650 \mathrm{mg}$ ) and polyphosphoric acid (PPA, 15.0 g) was added to a round bottom flask and stirred at $110^{\circ} \mathrm{C}$ for $6 \mathrm{~h}$. After the completion of the reaction, the residue was quenched with ice water and extracted into ethyl acetate. The organic phases were dried over anhydrous $\mathrm{Na}_{2} \mathrm{SO}_{4}$ and concentrated in vacuo. The crude product was purified by silica gel column chromatography (petroleum ether/EtOAc) to give the corresponding substituted indole S1.

According to a literature procedure, to the solution of indole S1 $(3.5 \mathrm{mmol}, 1.0$ equiv, $1.12 \mathrm{~g}$ ) and DMAP (0.7 mmol, 0.2 equiv, $85 \mathrm{mg})$ in dichoromethane $(7 \mathrm{ml})$ was added $\mathrm{Et}_{3} \mathrm{~N}$ (7 mmol, 2.0 equiv, $707 \mathrm{mg}$ ) and chloride (4.2 mmol, 1.2 equiv, 437 mg) at $0{ }^{\circ} \mathrm{C}$. The solution was warmed up to room temperature and stirred for overnight. The mixture was diluted with dichoromethane $(20 \mathrm{~mL})$. The organic and aqueous layers were separated. The aqueous layer was extracted with dichoromethane $(20 \mathrm{~mL} \times 2)$. The combined organic layer was washed with brine, dried over $\mathrm{Na}_{2} \mathrm{SO}_{4}$, filtered and concentrated in vacuo to give a residue, which was purified by flash chromatography and then recrystallized from n-hexane/EtOAc to afford the product 4a. The synthesis process of substrates $\mathbf{4 b - 4 g}$ is similar to $\mathbf{4 a}$.

\subsection{General Procedure for the Synthesis of Substrates $4 h^{[6,7,9]}$}

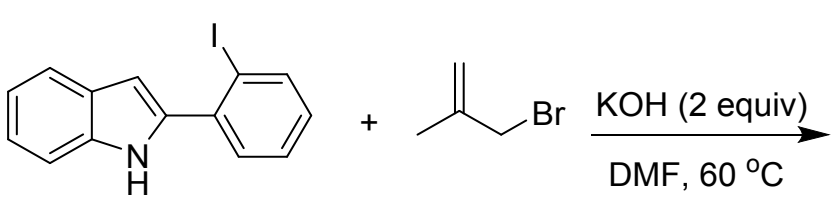

S1

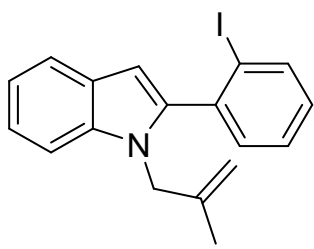

$4 h$

According to a literature procedure, a solution of $\mathbf{S} 1$ (3.0 mmol, 1.0 equiv, 955 $\mathrm{mg})$ in DMF (5 mL) and powdered $\mathrm{KOH}(3.9 \mathrm{mmol}, 1.3$ equiv, $218 \mathrm{mg}$ ) was stirred at $60{ }^{\circ} \mathrm{C}$ for $10 \mathrm{~min}$, cooled to room temperature, and treated with 
3-bromo-2-methylprop-1-ene (4.5 mmol, 1.5 equiv, $600 \mathrm{mg}$ ). The reaction mixture was stirred at $60{ }^{\circ} \mathrm{C}$ for $12-18 \mathrm{~h}$, poured onto ice and diluted with $15 \mathrm{~mL}$ of EtOAc. The combined organic layers were washed with $\mathrm{H}_{2} \mathrm{O}$, brine, dried $\mathrm{Na}_{2} \mathrm{SO}_{4}$, concentrated in vacuo and purified by chromatography on $\mathrm{SiO}_{2}$ (hexane) to afford the desired product $\mathbf{4 h}$.

2.6 General Procedure for the Synthesis of Substrates 2a-2[ [10, 11]



According to a literature procedure, to a cold $\left(-10^{\circ} \mathrm{C}\right)$ magnetically stirred solution of a 2-bromobenzaldehyde ( $5.0 \mathrm{mmol}, 1.0$ equiv, $920 \mathrm{mg})$ in dry ether $(20$ $\mathrm{mL}$ ) was added methylmagnesium bromide (5.0 mmol, 1.0 equiv) [prepared from magnesium (5.0 mmol, 1.0 equiv, $120 \mathrm{mg}$ ) and methyl bromomethane (5.0 mmol, 1.0 equiv, $475 \mathrm{mg}$ ) and a catalytic amount of iodine in $10 \mathrm{~mL}$ of dry ether]. The reaction mixture was stirred at $-10{ }^{\circ} \mathrm{C}$ for $3 \mathrm{~h}$. It was then poured into saturated aqueous $\mathrm{NH}_{4} \mathrm{Cl}$ solution and extracted with EtOAc $(3 \times 15 \mathrm{ml})$. The ethyl acetate extract was dried with $\mathrm{Na}_{2} \mathrm{SO}_{4}$. Evaporation of the solvent and purification of the residue over a silica gel column using petroleum ether/EtOAc as eluent furnished the 1-(2-bromophenyl)ethan-1-ol.

To a magnetically stirred solution of the 1-(2-bromophenyl)ethan-1-ol (5.0 mmol, 1.0 equiv, $1.0 \mathrm{~g})$ in dry $\mathrm{CH}_{2} \mathrm{Cl}_{2}(20 \mathrm{~mL})$ was added a homogeneous mixture of PCC (15.0 mmol, 3.0 equiv, $3.23 \mathrm{~g}$ ) and silica gel. The resulted reaction mixture was stirred at room temperature for $2 \mathrm{~h}$. The reaction mixture was then filtered through a short silica gel column and eluted with excess $\mathrm{CH}_{2} \mathrm{Cl}_{2}$. Evaporation of the solvent furnished the 1-(2-bromophenyl)ethan-1-one.

1-(2-bromophenyl)ethan-1-one (5.0 mmol, 1.0 equiv, $990 \mathrm{mg})$ was dissolved in pyridine $(10 \mathrm{~mL})$ and $\mathrm{SeO}_{2}(10.0 \mathrm{mmol}, 2.0$ equiv, $1.1 \mathrm{~g})$ was added. The reaction mixture was heated in an oil bath at $90{ }^{\circ} \mathrm{C}$ under nitrogen for $5 \mathrm{~h}$. The reaction was monitored by TLC. Upon completion, the reaction mixture was filtered, and the 
residue was washed with EtOAc $(3 \times 20 \mathrm{~mL})$. The combined filtrate was treated with $1 \mathrm{M} \mathrm{HCl}(40 \mathrm{~mL})$, the organic layer was separated, and the aqueous layer was re-extracted with EtOAc $(3 \times 20 \mathrm{~mL})$. The organic layers were combined and treated with $1 \mathrm{M} \mathrm{NaOH}(50 \mathrm{~mL})$, and the aqueous layer was separated. The aqueous layer was acidified with $1 \mathrm{M} \mathrm{HCl}$ to $\mathrm{pH} 1.5$. The mixture was extracted with EtOAc $(3 \times 50$ $\mathrm{mL}$ ). The combined organic layers were dried over $\mathrm{Na}_{2} \mathrm{SO}_{4}$ and concentrated. The crude products were further purified by column chromatography using petroleum ether /EtOAc (9:1 to 7:3) to afford 2a as a yellow solid in 35\% yield. The synthesis process of substrates $\mathbf{2 b - 2 l}$ is similar to $\mathbf{2 a}$.

\section{Typical Procedures}

\subsection{Typical Procedures for the synthesis of Isoquinolinedione-Fused}

\section{Benzocycloheptanones 3}

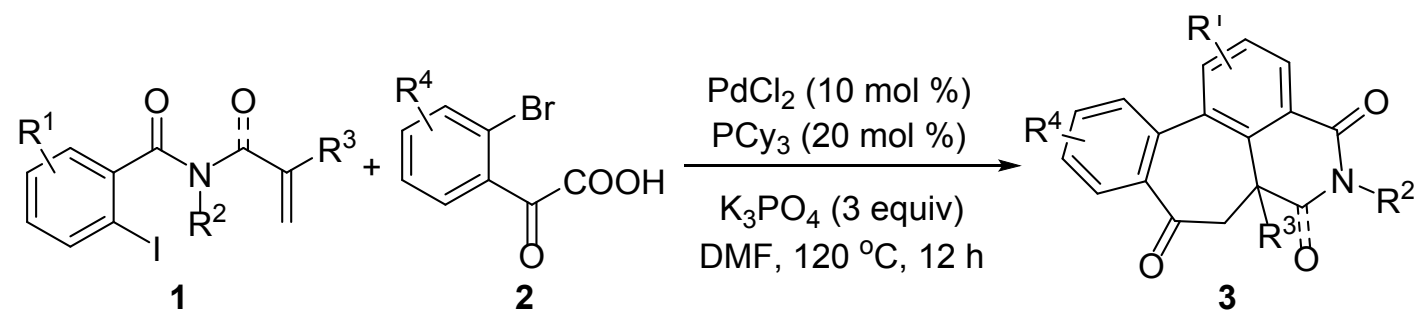

The mixture of $N$-benzyl-2-iodo- $N$-methacryloylbenzamide 1a $(0.2 \mathrm{mmol}, 1.0$ equiv, $81 \mathrm{mg}$ ), 2-(2-bromoaryl)-2-oxoacetic acids $2 \mathrm{a}$ ( $0.3 \mathrm{mmol}, 1.5$ equiv, $64.8 \mathrm{mg})$, $\mathrm{PdCl}_{2}(0.02 \mathrm{mmol}, 10 \mathrm{~mol} \%, 3.4 \mathrm{mg}), \mathrm{PCy}_{3}(0.04 \mathrm{mmol}, 20 \mathrm{~mol} \%, 11.2 \mathrm{mg})$, and $\mathrm{K}_{3} \mathrm{PO}_{4}(0.6 \mathrm{mmol}, 3.0$ equiv, $127.2 \mathrm{mg})$ in DMF (2 mL) was stirred under nitrogen atmosphere at $120{ }^{\circ} \mathrm{C}$ for $12 \mathrm{~h}$ (oil bath temperature). After the completion of the reaction (monitored by TLC), the reaction mixture was filtered and the filtrate was washed by $\mathrm{H}_{2} \mathrm{O}$ and saturated salt solution and then evaporated under reduced pressure, the crude product was purified by column chromatography (eluent: petroleum ether/EtOAc $=10: 1)$ to provide the desired product 3aa $(75 \%, 57.15 \mathrm{mg})$. 


\subsection{General Procedure for the Synthesis of Indolo[2,1-a]isoquinolinone-Fused}

\section{Benzocycloheptanones 5}<smiles>[R][Y]1cccc2cc(-c3ccccc3I)n(C(=O)C([R])=C)c12</smiles>

4<smiles>O=C(O)c1ccccc1Br</smiles>

2

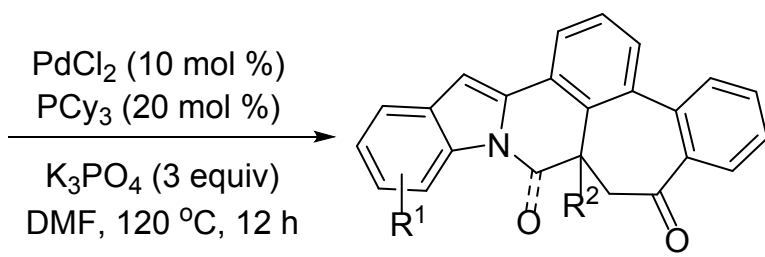

5

To a Schlenk tube were added alkene-tethered aryl iodides $4 \mathbf{a}(0.2 \mathrm{mmol}, 1.0$ equiv, $77.4 \mathrm{mg})$, 2-(2-bromoaryl)-2-oxoacetic acids $2 \mathbf{a}(0.3 \mathrm{mmol}, 1.5$ equiv, 64.8 $\mathrm{mg}), \mathrm{PdCl}_{2}(0.02 \mathrm{mmol}, 10 \mathrm{~mol} \%, 3.4 \mathrm{mg}), \mathrm{PCy}_{3}(0.04 \mathrm{mmol}, 20 \mathrm{~mol} \%, 11.2 \mathrm{mg})$, and $\mathrm{K}_{3} \mathrm{PO}_{4}(0.6 \mathrm{mmol}, 3.0$ equiv, $127.2 \mathrm{mg})$ in $\mathrm{DMF}(2 \mathrm{~mL})$ was stirred under nitrogen atmosphere at $120^{\circ} \mathrm{C}$ for $12 \mathrm{~h}$ (oil bath temperature). After the completion of the reaction (monitored by TLC), the reaction mixture was filtered and the filtrate was washed by $\mathrm{H}_{2} \mathrm{O}$ and saturated salt solution and then evaporated under reduced pressure, the crude product was purified by column chromatography (eluent: petroleum ether/EtOAc $=10: 1)$ to provide the desired product 5aa $(78 \%, 56.94 \mathrm{mg})$.

\subsection{General Procedure for the Synthesis of 3aa from $1 \mathrm{mmol}$ Scale of 1a}

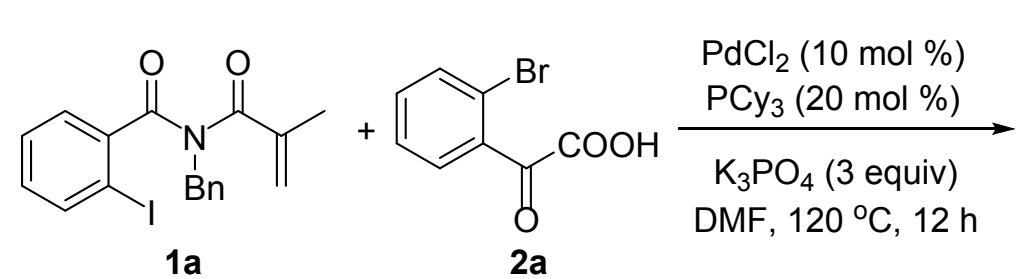<smiles></smiles>

To Schlenk tube were added $N$-benzyl-2-iodo- $N$-methacryloylbenzamide 1a (1.0 mmol, 1.0 equiv, $405 \mathrm{mg})$, 2-(2-bromoaryl)-2-oxoacetic acids $\mathbf{2 a}$ (1.5 mmol, 1.5 equiv, $324 \mathrm{mg}), \mathrm{PdCl}_{2}(0.1 \mathrm{mmol}, 10 \mathrm{~mol} \%, 17 \mathrm{mg}), \mathrm{PCy}_{3}(0.04 \mathrm{mmol}, 20 \mathrm{~mol} \%, 56$ $\mathrm{mg})$, and $\mathrm{K}_{3} \mathrm{PO}_{4}(3.0 \mathrm{mmol}, 3.0$ equiv, $636 \mathrm{mg})$ in DMF $(8 \mathrm{~mL})$ was stirred under nitrogen atmosphere at $120{ }^{\circ} \mathrm{C}$ for $12 \mathrm{~h}$ (oil bath temperature). After the completion of the reaction (monitored by TLC), the reaction mixture was filtered and the filtrate was washed by $\mathrm{H}_{2} \mathrm{O}$ and saturated salt solution and then evaporated under reduced pressure, the crude product was purified by column chromatography (eluent: petroleum ether/EtOAc $=10: 1)$ to provide the desired product 3aa $(67 \%, 255.3 \mathrm{mg})$. 


\section{Isotope experiment}
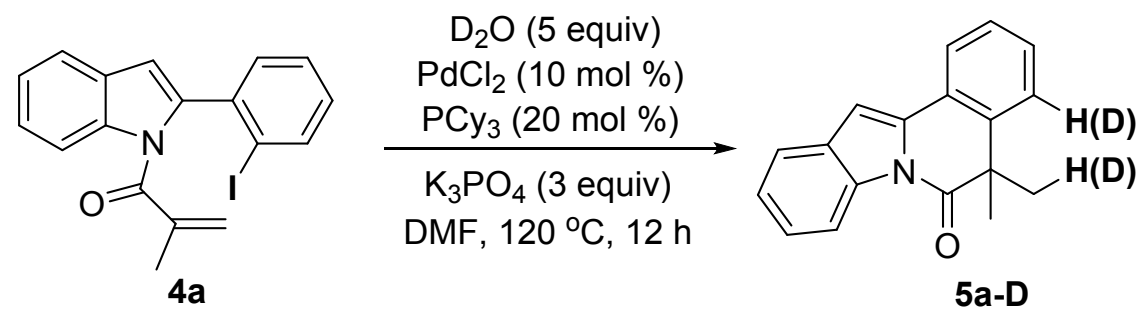

To a Schlenk tube were added alkene-tethered aryl iodides $4 \mathbf{a}(0.2 \mathrm{mmol}, 1.0$ equiv, $77.4 \mathrm{mg}$ ), $\mathrm{D}_{2} \mathrm{O}$ (1.0 mmol, 5.0 equiv, $\left.20 \mathrm{mg}\right), \mathrm{PdCl}_{2}$ (0.02 mmol, $10 \mathrm{~mol} \%, 3.4$ $\mathrm{mg}), \mathrm{PCy}_{3}(0.04 \mathrm{mmol}, 20 \mathrm{~mol} \%, 11.2 \mathrm{mg})$, and $\mathrm{K}_{3} \mathrm{PO}_{4}(0.6 \mathrm{mmol}, 3.0$ equiv, 127.2 $\mathrm{mg}$ ) in DMF (2 mL) was stirred under nitrogen atmosphere at $120^{\circ} \mathrm{C}$ for $12 \mathrm{~h}$ (oil bath temperature). After the completion of the reaction (monitored by TLC), the reaction mixture was filtered and the filtrate was washed by $\mathrm{H}_{2} \mathrm{O}$ and saturated salt solution and then evaporated under reduced pressure, the crude product was purified by column chromatography (eluent: petroleum ether/EtOAc $=100: 1$ ) to provide the desired product 5a-D $(36 \%, 19.1 \mathrm{mg})$.<smiles>CC1(C[Hg])C(=O)n2c(cc3ccccc32)-c2cccc([Hg])c21</smiles>

5,5-dimethylindolo[2,1-a]isoquinolin-6(5H)-one (5a-D): oil liquid, isolated yield 36\% (19.1 mg); ${ }^{1} \mathbf{H}$ NMR (500 MHz, $\left.\mathrm{CDCl}_{3}\right) \delta=8.56(\mathrm{~d}, J=8.0 \mathrm{~Hz}, 1 \mathrm{H}), 7.84(\mathrm{dd}, J$ $=7.0,1.5 \mathrm{~Hz}, 1 \mathrm{H}), 7.59(\mathrm{~d}, J=7.5 \mathrm{~Hz}, 1 \mathrm{H}), 7.45(\mathrm{~d}, J=7.0 \mathrm{~Hz}, 0.5 \mathrm{H}), 7.36(\mathrm{t}, J=7.5$ $\mathrm{Hz}, 3 \mathrm{H}), 7.31(\mathrm{t}, J=7.5 \mathrm{~Hz}, 1 \mathrm{H}), 7.02(\mathrm{~s}, 1 \mathrm{H}), 1.70-1.60(\mathrm{~m}, 5.21 \mathrm{H})$.<smiles>CC1(C)C(=O)n2c(cc3ccccc32)-c2ccccc21</smiles>

5,5-dimethylindolo[2,1-a]isoquinolin-6(5H)-one (5a): oil liquid, isolated yield $42 \%$ $(21.9 \mathrm{mg}) ;{ }^{1} \mathbf{H}$ NMR $\left(500 \mathrm{MHz}, \mathrm{CDCl}_{3}\right) \delta=8.58(\mathrm{~d}, J=8.0 \mathrm{~Hz}, 1 \mathrm{H}), 7.85(\mathrm{dd}, J=7.0$, $1.5 \mathrm{~Hz}, 1 \mathrm{H}), 7.61(\mathrm{~d}, J=7.5 \mathrm{~Hz}, 1 \mathrm{H}), 7.47-7.45(\mathrm{~m}, 1 \mathrm{H}), 7.40-7.35(\mathrm{~m}, 3 \mathrm{H})$, 7.34-7.31 (m, 1H), $7.03(\mathrm{~s}, 1 \mathrm{H}), 1.72(\mathrm{~s}, 6 \mathrm{H})$. 
${ }^{1} \mathrm{H}$ NMR of 5a-D $\left(500 \mathrm{MHz}, \mathrm{CDCl}_{3}\right)$

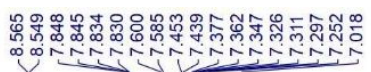

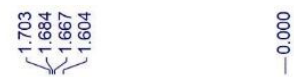

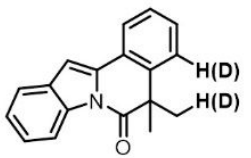

${ }^{1} \mathrm{H} \mathrm{NMR}$ of $5 \mathbf{a}\left(500 \mathrm{MHz}, \mathrm{CDCl}_{3}\right)$



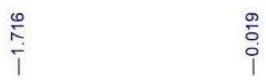



\section{Crystal Culture Procedure of Product 3aa}

To a round-bottom flask $(25 \quad \mathrm{~mL})$ was added 5-benzyl-6a-methyl-6a,7-dihydrobenzo[4,5]cyclohepta[1,2,3-de]isoquinoline-4,6,8(5 H)-trione 3aa $(10 \mathrm{mg})$. Dichloromethane $(1.0 \mathrm{~mL})$ were added slowly to make it dissolve completely. Then petroleum ether $(5.0 \mathrm{~mL})$ were added. Finally, the round-bottom flask was sealed with a rubber stopper, and connected the air with a syringe needle. Putting the flask in a dry and ventilated place to make the organic solvent volatilize slowly. After a few days, the crystal of 3aa were separated out.

\section{Characterization Data}<smiles>CC12CC(=O)c3ccccc3-c3cccc(c31)C(=O)N(Cc1ccccc1)C2=O</smiles>

5-benzyl-6a-methyl-6a,7-dihydrobenzo[4,5]cyclohepta[1,2,3-de]isoquinoline-4,6,8 (5H)-trione (3aa): white solid, isolated yield 75\% (57.15 mg), 70\% (53.34 mg) ( $N$-benzyl-2-bromo- $N$-methacryloylbenzamide was used) (eluent: petroleum ether/EtOAc $=10: 1) ; \mathrm{mp}: 164.6-166.8^{\circ} \mathrm{C}$ (uncorrected); ${ }^{1} \mathbf{H}$ NMR $\left(500 \mathrm{MHz}, \mathrm{CDCl}_{3}\right)$ $\delta=8.39(\mathrm{~d}, J=7.5 \mathrm{~Hz}, 1 \mathrm{H}), 7.77(\mathrm{~d}, J=8.0 \mathrm{~Hz}, 1 \mathrm{H}), 7.71-7.66(\mathrm{~m}, 2 \mathrm{H}), 7.60(\mathrm{t}, J=$ $7.5 \mathrm{~Hz}, 1 \mathrm{H}), 7.52$ (t, $J=7.5 \mathrm{~Hz}, 1 \mathrm{H}), 7.46-7.41(\mathrm{~m}, 3 \mathrm{H}), 7.30$ (t, $J=7.3 \mathrm{~Hz}, 2 \mathrm{H}), 7.25$ (t, $J=7.0 \mathrm{~Hz}, 1 \mathrm{H}), 5.23(\mathrm{~s}, 2 \mathrm{H}), 3.65(\mathrm{~d}, J=19.5 \mathrm{~Hz}, 1 \mathrm{H}), 3.14(\mathrm{~d}, J=19.5 \mathrm{~Hz}, 1 \mathrm{H})$, $1.52(\mathrm{~s}, 3 \mathrm{H}) ;{ }^{13} \mathrm{C}$ NMR $\left(125 \mathrm{MHz}, \mathrm{CDCl}_{3}\right) \delta=200.7,175.4,163.4,139.8,138.6$, $137.9,137.6,137.4,136.7,133.4,130.0,129.6,128.8$ (2C), 128.7, 128.5 (2C), 127.6, 123.7, 56.6, 43.8, 43.7, 23.5. HRMS (ESI-TOF) m/z: $[\mathrm{M}+\mathrm{H}]^{+}$Calcd for $\mathrm{C}_{25} \mathrm{H}_{20} \mathrm{NO}_{3}{ }^{+}$ 382.1438; found 382.1440 .<smiles>Cc1ccc(CN2C(=O)c3cccc4c3C(C)(CC(=O)c3ccccc3-4)C2=O)cc1</smiles>

6a-methyl-5-(4-methylbenzyl)-6a,7-dihydrobenzo[4,5]cyclohepta[1,2,3-de]isoquin oline-4,6,8(5H)-trione (3ba): white solid, isolated yield 65\% (51.35 mg) (eluent: petroleum ether/EtOAc $=10: 1), \mathrm{mp}: 159.8-162.0{ }^{\circ} \mathrm{C}$ (uncorrected); ${ }^{1} \mathbf{H}$ NMR $(500$ 
$\left.\mathrm{MHz}, \mathrm{CDCl}_{3}\right) \delta=8.38(\mathrm{~d}, J=7.5 \mathrm{~Hz}, 1 \mathrm{H}), 7.76(\mathrm{~d}, J=7.5 \mathrm{~Hz}, 1 \mathrm{H}), 7.70-7.66(\mathrm{~m}$, 2H), 7.59 (t, $J=7.5 \mathrm{~Hz}, 1 \mathrm{H}), 7.51$ (t, $J=7.5 \mathrm{~Hz}, 1 \mathrm{H}), 7.45$ (d, $J=8.0 \mathrm{~Hz}, 1 \mathrm{H}), 7.32$ (d, $J=7.5 \mathrm{~Hz}, 2 \mathrm{H}), 7.10(\mathrm{~d}, J=8.0 \mathrm{~Hz}, 2 \mathrm{H}), 5.18(\mathrm{~s}, 2 \mathrm{H}), 3.64(\mathrm{~d}, J=19.5 \mathrm{~Hz}, 1 \mathrm{H})$, $3.13(\mathrm{~d}, J=19.5 \mathrm{~Hz}, 1 \mathrm{H}), 2.30(\mathrm{~s}, 3 \mathrm{H}), 1.51(\mathrm{~s}, 3 \mathrm{H}) ;{ }^{13} \mathbf{C ~ N M R}\left(125 \mathrm{MHz}, \mathrm{CDCl}_{3}\right) \delta=$ 200.7, 175.3, 163.3, 139.8, 138.6, 137.8, 137.6, 137.4, 137.2, 133.7, 133.3, 123.0, $129.5,129.1,128.8,128.7$ (2C), 128.5, 123.7, 56.5, 43.7, 43.4, 23.4, 21.1. HRMS (ESI-TOF) m/z: [M+ H] $]^{+}$Calcd for $\mathrm{C}_{26} \mathrm{H}_{22} \mathrm{NO}_{3}{ }^{+}$396.1594; found 396.1596.<smiles>CC12CC(=O)c3ccccc3-c3cccc(c31)C(=O)N(Cc1ccc(F)cc1)C2=O</smiles>

\section{5-(4-fluorobenzyl)-6a-methyl-6a,7-dihydrobenzo[4,5]cyclohepta[1,2,3-de]isoquin}

oline-4,6,8(5H)-trione (3ca): white solid, isolated yield 75\% (59.85 mg) (eluent: petroleum ether/EtOAc $=10: 1) ; \mathrm{mp}: 136.4-138.6{ }^{\circ} \mathrm{C}$ (uncorrected); ${ }^{1} \mathbf{H}$ NMR (500 $\left.\mathrm{MHz}, \mathrm{CDCl}_{3}\right) \delta=8.38(\mathrm{~d}, J=8.0 \mathrm{~Hz}, 1 \mathrm{H}), 7.77(\mathrm{~d}, J=7.5 \mathrm{~Hz}, 1 \mathrm{H}), 7.72-7.67(\mathrm{~m}$, 2H), $7.61(\mathrm{t}, J=7.0 \mathrm{~Hz}, 1 \mathrm{H}), 7.52(\mathrm{t}, J=7.5 \mathrm{~Hz}, 1 \mathrm{H}), 7.46-7.43(\mathrm{~m}, 3 \mathrm{H}), 6.98(\mathrm{t}, J=$ $9.0 \mathrm{~Hz}, 2 \mathrm{H}), 5.18$ (s, 2H), $3.64(\mathrm{~d}, J=19.5 \mathrm{~Hz}, 1 \mathrm{H}), 3.12(\mathrm{~d}, J=19.0 \mathrm{~Hz}, 1 \mathrm{H}), 1.51(\mathrm{~s}$, $3 \mathrm{H}) ;{ }^{13} \mathrm{C}$ NMR $\left(125 \mathrm{MHz}, \mathrm{CDCl}_{3}\right) \delta=200.6,175.4,163.4,162.2\left(\mathrm{~d}, \mathrm{C}-\mathrm{F},{ }^{1} J_{\mathrm{C}-\mathrm{F}}=\right.$ $244.6 \mathrm{~Hz}), 139.8,138.5,137.9,137.7,137.4,133.4,132.5\left(\mathrm{~d}, \mathrm{C}-\mathrm{F},{ }^{4} J_{\mathrm{C}-\mathrm{F}}=3.3 \mathrm{~Hz}\right)$, $130.7\left(\mathrm{~d}, \mathrm{C}-\mathrm{F},{ }^{3} J_{\mathrm{C}-\mathrm{F}}=8.0 \mathrm{~Hz}\right), 130.0,129.6,128.9,128.8,128.7,123.6,115.3(\mathrm{~d}, \mathrm{C}-\mathrm{F}$, $\left.{ }^{2} J_{\mathrm{C}-\mathrm{F}}=21.3 \mathrm{~Hz}\right), 56.5,43.8,43.0,23.5$. HRMS (ESI-TOF) $\mathrm{m} / \mathrm{z}:[\mathrm{M}+\mathrm{H}]^{+}$Calcd for $\mathrm{C}_{25} \mathrm{H}_{19} \mathrm{FNO}_{3}{ }^{+} 400.1343$; found 400.1345 .<smiles>CN1C(=O)c2cccc3c2C(C)(CC(=O)c2ccccc2-3)C1=O</smiles>

5,6a-dimethyl-6a,7-dihydrobenzo[4,5]cyclohepta[1,2,3-de]isoquinoline-4,6,8(5H)trione (3da): white solid, isolated yield 72\% (43.92 mg) (eluent: petroleum ether/EtOAc = 10:1); mp: $177.8-179.7^{\circ} \mathrm{C}$ (uncorrected); ${ }^{1} \mathbf{H}$ NMR $\left(500 \mathrm{MHz}, \mathrm{CDCl}_{3}\right)$ $\delta=8.38(\mathrm{~d}, J=7.5 \mathrm{~Hz}, 1 \mathrm{H}), 7.78(\mathrm{~d}, J=7.5 \mathrm{~Hz}, 1 \mathrm{H}), 7.73-7.68(\mathrm{~m}, 2 \mathrm{H}), 7.62(\mathrm{t}, J=$ 
$8.0 \mathrm{~Hz}, 1 \mathrm{H}), 7.53$ (t, $J=7.0 \mathrm{~Hz}, 1 \mathrm{H}), 7.48(\mathrm{~d}, J=8.0 \mathrm{~Hz}, 1 \mathrm{H}), 3.66(\mathrm{~d}, J=19.0 \mathrm{~Hz}$, 1H), $3.42(\mathrm{~s}, 3 \mathrm{H}), 3.15(\mathrm{~d}, J=19.5 \mathrm{~Hz}, 1 \mathrm{H}), 1.53$ (s, 3H); ${ }^{13} \mathbf{C}$ NMR (125 MHz, $\left.\mathrm{CDCl}_{3}\right) \delta=200.6,175.7,163.7,139.8,138.6,137.9,137.5,137.4,133.3,130.0$, 129.3, 128.8, 128.7 (2C), 123.5, 56.4, 43.7, 27.4, 23.5. HRMS (ESI-TOF) m/z: [M + $\mathrm{H}]^{+}$Calcd for $\mathrm{C}_{19} \mathrm{H}_{16} \mathrm{NO}_{3}{ }^{+}$306.1125; found 306.1126.

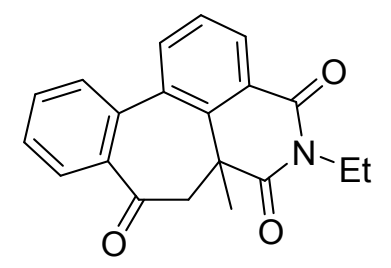

\section{5-ethyl-6a-methyl-6a,7-dihydrobenzo[4,5]cyclohepta[1,2,3-de]isoquinoline-4,6,8(}

$\mathbf{5 H}$ )-trione (3ea): white solid, isolated yield 66\% (42.11 mg) (eluent: petroleum ether/EtOAc = 10:1); mp: 183.4-188.0 ${ }^{\circ} \mathrm{C}$ (uncorrected); ${ }^{1} \mathbf{H} \mathbf{N M R}\left(500 \mathrm{MHz}, \mathrm{CDCl}_{3}\right)$ $\delta=8.39(\mathrm{~d}, J=7.5 \mathrm{~Hz}, 1 \mathrm{H}), 7.78(\mathrm{~d}, J=7.5 \mathrm{~Hz}, 1 \mathrm{H}), 7.72-7.67(\mathrm{~m}, 2 \mathrm{H}), 7.62(\mathrm{t}, J=$ $8.0 \mathrm{~Hz}, 1 \mathrm{H}), 7.53(\mathrm{t}, J=8.0 \mathrm{~Hz}, 1 \mathrm{H}), 7.47(\mathrm{~d}, J=8.0 \mathrm{~Hz}, 1 \mathrm{H}), 4.11-4.08(\mathrm{~m}, 2 \mathrm{H})$, $3.66(\mathrm{~d}, J=19.0 \mathrm{~Hz}, 1 \mathrm{H}), 3.15(\mathrm{~d}, J=19.5 \mathrm{~Hz}, 1 \mathrm{H}), 1.53(\mathrm{~s}, 3 \mathrm{H}), 1.25$ (t, $J=7.5 \mathrm{~Hz}$, $3 \mathrm{H}) ;{ }^{13} \mathbf{C}$ NMR $\left(125 \mathrm{MHz}, \mathrm{CDCl}_{3}\right) \delta=200.8,175.2,163.2,139.9,138.7,137.9$, 137.5, 133.4, 130.0, 129.4, 128.8, 128.7 (2C), 123.8, 56.5, 43.6, 35.9, 23.5, 13.0. HRMS (ESI-TOF) m/z: $[\mathrm{M}+\mathrm{H}]^{+}$Calcd for $\mathrm{C}_{20} \mathrm{H}_{18} \mathrm{NO}_{3}{ }^{+}$320.1281; found 320.1284.<smiles>CC12CC(=O)c3ccccc3-c3cccc(c31)C(=O)N(c1ccccc1)C2=O</smiles>

6a-methyl-5-phenyl-6a,7-dihydrobenzo[4,5] cyclohepta[1,2,3-de]isoquinoline-4,6,8 (5H)-trione (3fa): white solid, isolated yield 71\% (52.11 mg) (eluent: petroleum ether/EtOAc = 10:1); mp: 199.3-205.0 ${ }^{\circ} \mathrm{C}$ (uncorrected); ${ }^{1} \mathbf{H}$ NMR (500 MHz, $\left.\mathrm{CDCl}_{3}\right)$ $\delta=8.41(\mathrm{~d}, J=7.5 \mathrm{~Hz}, 1 \mathrm{H}), 7.79-7.76(\mathrm{~m}, 2 \mathrm{H}), 7.71(\mathrm{t}, J=7.5 \mathrm{~Hz}, 1 \mathrm{H}), 7.64(\mathrm{t}, J=$ $8.0 \mathrm{~Hz}, 1 \mathrm{H}), 7.55-7.44(\mathrm{~m}, 5 \mathrm{H}), 7.21(\mathrm{~d}, J=7.5 \mathrm{~Hz}, 2 \mathrm{H}), 3.70(\mathrm{~d}, J=19.5 \mathrm{~Hz}, 1 \mathrm{H})$, $3.32(\mathrm{~d}, J=19.0 \mathrm{~Hz}, 1 \mathrm{H}), 1.63(\mathrm{~s}, 3 \mathrm{H}) ;{ }^{13} \mathbf{C} \mathbf{N M R}\left(125 \mathrm{MHz}, \mathrm{CDCl}_{3}\right) \delta=200.6$, 175.6, 163.6, 140.0, 138.6, 138.1, 137.9, 137.4, 134.9, 133.4, 130.1, 129.7, 129.4, 128.9 (2C), 128.8, 128.7, 128.2, 123.8, 56.5, 44.3, 23.7. HRMS (ESI-TOF) m/z: [M + $\mathrm{H}]^{+}$Calcd for $\mathrm{C}_{24} \mathrm{H}_{18} \mathrm{NO}_{3}{ }^{+} 368.1281$; found 368.1283 . 
<smiles>C=CCN1C(=O)c2cccc3c2C(C)(CC(=O)c2ccccc2-3)C1=O</smiles>

5-allyl-6a-methyl-6a,7-dihydrobenzo[4,5]cyclohepta[1,2,3-de]isoquinoline-4,6,8(5 $\boldsymbol{H}$ )-trione (3ga): white solid, isolated yield 30\% (19.86 mg) (eluent: petroleum ether/EtOAc $=10: 1) ; \mathrm{mp}: 145.5-147.7{ }^{\circ} \mathrm{C}$ (uncorrected); ${ }^{1} \mathbf{H}$ NMR $\left(500 \mathrm{MHz}, \mathrm{CDCl}_{3}\right)$ $\delta=8.40(\mathrm{~d}, J=7.5 \mathrm{~Hz}, 1 \mathrm{H}), 7.78(\mathrm{~d}, J=7.5 \mathrm{~Hz}, 1 \mathrm{H}), 7.73-7.68(\mathrm{~m}, 2 \mathrm{H}), 7.62(\mathrm{t}, J=$ $8.0 \mathrm{~Hz}, 1 \mathrm{H}), 7.53$ (t, $J=7.5 \mathrm{~Hz}, 1 \mathrm{H}), 7.47$ (d, $J=7.8 \mathrm{~Hz}, 1 \mathrm{H}), 5.95-5.87$ (m, 1H), $5.26(\mathrm{~d}, J=17.0 \mathrm{~Hz}, 1 \mathrm{H}), 5.20(\mathrm{~d}, J=10.5 \mathrm{~Hz}, 1 \mathrm{H}), 4.65(\mathrm{~d}, J=5.0 \mathrm{~Hz}, 2 \mathrm{H}), 3.66(\mathrm{~d}$, $J=19.0 \mathrm{~Hz}, 1 \mathrm{H}), 3.16(\mathrm{~d}, J=19.0 \mathrm{~Hz}, 1 \mathrm{H}), 1.54(\mathrm{~s}, 3 \mathrm{H}) ;{ }^{13} \mathbf{C}$ NMR (125 MHz, $\left.\mathrm{CDCl}_{3}\right) \delta=200.8,175.1,163.2,139.9,138.6,138.0,137.7,137.5,133.4,131.5$, $130.1,129.6,128.9,128.8,128.8,123.6,117.8,56.6,43.8,42.6,23.6$. HRMS (ESI-TOF) m/z: [M + H] $]^{+}$Calcd for $\mathrm{C}_{21} \mathrm{H}_{18} \mathrm{NO}_{3}{ }^{+}$332.1281; found 332.1283.<smiles>O=C1C[C@]2(P)C(=O)N(Cc3ccccc3)C(=O)c3cccc(c32)-c2ccccc21</smiles>

5-benzyl-6a-phenyl-6a,7-dihydrobenzo[4,5]cyclohepta[1,2,3-de]isoquinoline-4,6,8 (5H)-trione (3ha): white solid, isolated yield 48\% (41.18 mg) (eluent: petroleum ether/EtOAc = 10:1); mp: 197.6-201.0 ${ }^{\circ} \mathrm{C}$ (uncorrected); ${ }^{1} \mathbf{H}$ NMR $\left(500 \mathrm{MHz}, \mathrm{CDCl}_{3}\right)$ $\delta=8.47(\mathrm{dd}, J=6.0,3.0 \mathrm{~Hz}, 1 \mathrm{H}), 7.69(\mathrm{~s}, 2 \mathrm{H}), 7.57-7.55(\mathrm{~m}, 1 \mathrm{H}), 7.30-7.27(\mathrm{~m}, 3 \mathrm{H})$, 7.22-7.18 (m, 4H), 7.00-6.96 (m, 6H), $5.21(\mathrm{~d}, J=14.0 \mathrm{~Hz}, 1 \mathrm{H}), 5.06(\mathrm{~d}, J=14.0 \mathrm{~Hz}$, 1H), $4.46(\mathrm{~d}, J=19.5 \mathrm{~Hz}, 1 \mathrm{H}), 3.33$ (d, $J=20.0 \mathrm{~Hz}, 1 \mathrm{H}) ;{ }^{13} \mathrm{C}$ NMR (125 MHz, $\left.\mathrm{CDCl}_{3}\right) \delta=200.2,172.8,163.4,140.2,138.8,138.2,137.9,137.6,137.2,136.5$, 132.8, 130.1, 129.3, 129.1, 128.4 (2C), 128.2 (2C), 128.1, 127.5 (2C), 127.4, 124.6, 54.6, 51.8, 44.1. HRMS (ESI-TOF) m/z: $[\mathrm{M}+\mathrm{H}]^{+}$Calcd for $\mathrm{C}_{30} \mathrm{H}_{22} \mathrm{NO}_{3}{ }^{+}$444.1594; found 444.1594 . 
<smiles>Cc1ccc2c3c1C(=O)N(Cc1ccccc1)C(=O)C3(C)CC(=O)c1ccccc1-2</smiles>

5-benzyl-3,6a-dimethyl-6a,7-dihydrobenzo[4,5]cyclohepta[1,2,3-de]isoquinoline-4 , $\mathbf{6 , 8}(\mathbf{5 H})$-trione (3ia): white solid, isolated yield 46\% (36.34 mg) (eluent: petroleum ether/EtOAc $=10: 1) ; \mathrm{mp}: 169.1-171.3{ }^{\circ} \mathrm{C}$ (uncorrected); ${ }^{1} \mathbf{H}$ NMR $\left(500 \mathrm{MHz}, \mathrm{CDCl}_{3}\right)$ $\delta=7.78(\mathrm{~d}, J=7.5 \mathrm{~Hz}, 1 \mathrm{H}), 7.66(\mathrm{t}, J=7.5 \mathrm{~Hz}, 1 \mathrm{H}), 7.53-7.48(\mathrm{~m}, 2 \mathrm{H}), 7.42-7.39(\mathrm{~m}$, 4H), $7.30(\mathrm{t}, J=7.5 \mathrm{~Hz}, 2 \mathrm{H}), 7.25(\mathrm{~d}, J=6.5 \mathrm{~Hz}, 1 \mathrm{H}), 5.21(\mathrm{~s}, 2 \mathrm{H}), 3.70(\mathrm{~d}, J=19.0$ Hz, 1H), $3.11(\mathrm{~d}, J=19.5 \mathrm{~Hz}, 1 \mathrm{H}), 2.83$ (s, 3H), 1.50 (s, 3H); ${ }^{13} \mathrm{C}$ NMR (125 MHz, $\left.\mathrm{CDCl}_{3}\right) \delta=200.7,175.2,163.8,143.3,141.2,139.5,137.0,136.9,136.7,135.9$, $133.5,132.9,130.1,128.5$ (3C), 127.5, 122.0, 56.4, 43.9, 43.6, 24.5, 23.8. HRMS (ESI-TOF) m/z: [M + H] ${ }^{+}$Calcd for $\mathrm{C}_{26} \mathrm{H}_{22} \mathrm{NO}_{3}{ }^{+}$396.1594; found 396.1596 .<smiles>CC12CC(=O)c3ccccc3-c3ccc(F)c(c31)C(=O)N(Cc1ccccc1)C2=O</smiles>

\section{5-benzyl-3-fluoro-6a-methyl-6a,7-dihydrobenzo $[4,5]$ cyclohepta[1,2,3-de]isoquino} line-4,6,8(5H)-trione (3ja): white solid, isolated yield 55\% (43.89 mg) (eluent: petroleum ether/EtOAc $=10: 1) ; \mathrm{mp}: 174.9-175.9{ }^{\circ} \mathrm{C}$ (uncorrected); ${ }^{1} \mathbf{H}$ NMR $(500$ $\left.\mathrm{MHz}, \mathrm{CDCl}_{3}\right) \delta=8.06(\mathrm{~d}, J=7.0 \mathrm{~Hz}, 1 \mathrm{H}), 7.74(\mathrm{dd}, J=42.5,7.0 \mathrm{~Hz}, 2 \mathrm{H}), 7.56(\mathrm{t}, J=$ $6.5 \mathrm{~Hz}, 1 \mathrm{H}), 7.43(\mathrm{dd}, J=14.5,7.5 \mathrm{~Hz}, 4 \mathrm{H}), 7.31-7.26(\mathrm{~m}, 3 \mathrm{H}), 5.22(\mathrm{~s}, 2 \mathrm{H}), 3.64$ (d, $J=19.0 \mathrm{~Hz}, 1 \mathrm{H}), 3.13(\mathrm{~d}, J=19.5 \mathrm{~Hz}, 1 \mathrm{H}), 1.49$ (s, 3H); ${ }^{13} \mathbf{C}$ NMR (125 MHz, $\left.\mathrm{CDCl}_{3}\right) \delta=200.3,175.2,162.5,162.0\left(\mathrm{~d}, \mathrm{C}-\mathrm{F},{ }^{1} J_{\mathrm{C}-\mathrm{F}}=247.6 \mathrm{~Hz}\right), 140.7\left(\mathrm{~d}, \mathrm{C}-\mathrm{F},{ }^{3} J_{\mathrm{C}-\mathrm{F}}\right.$ $=7.8 \mathrm{~Hz}), 137.4(2 \mathrm{C}), 136.4,135.8\left(\mathrm{~d}, \mathrm{C}-\mathrm{F},{ }^{4} J_{\mathrm{C}-\mathrm{F}}=2.5 \mathrm{~Hz}\right), 133.6,129.9,129.4$, $128.9,128.6,128.6,127.7,126.0\left(\mathrm{~d}, \mathrm{C}-\mathrm{F},{ }^{3} J_{\mathrm{C}-\mathrm{F}}=7.6 \mathrm{~Hz}\right), 124.6\left(\mathrm{~d}, \mathrm{C}-\mathrm{F},{ }^{2} J_{\mathrm{C}-\mathrm{F}}=22.5\right.$ Hz), $115.5\left(\mathrm{~d}, \mathrm{C}-\mathrm{F},{ }^{2} J_{\mathrm{C}-\mathrm{F}}=23.0 \mathrm{~Hz}\right), 56.5,43.9,43.6,23.6$. HRMS (ESI-TOF) $\mathrm{m} / \mathrm{z}$ : $[\mathrm{M}+\mathrm{H}]^{+}$Calcd for $\mathrm{C}_{25} \mathrm{H}_{19} \mathrm{FNO}_{3}{ }^{+} 400.1343$; found 400.1345 . 
<smiles>Cc1cc2c3c(c1)-c1ccccc1C(=O)CC3(C)C(=O)N(Cc1ccccc1)C2=O</smiles>

5-benzyl-2,6a-dimethyl-6a,7-dihydrobenzo[4,5]cyclohepta[1,2,3-de]isoquinoline-4 , $\mathbf{6 , 8}(\mathbf{5 H})$-trione (3ka): white solid, isolated yield 73\% (57.67 mg) (eluent: petroleum ether/EtOAc = 10:1); mp: 198.4-199.8 ${ }^{\circ} \mathrm{C}$ (uncorrected); ${ }^{1} \mathbf{H}$ NMR $\left(500 \mathrm{MHz}, \mathrm{CDCl}_{3}\right)$ $\delta=8.19(\mathrm{~d}, J=1.0 \mathrm{~Hz}, 1 \mathrm{H}), 7.74(\mathrm{dd}, J=8.0,1.5 \mathrm{~Hz}, 1 \mathrm{H}), 7.66(\mathrm{td}, J=7.5,1.5 \mathrm{~Hz}$, 1H), 7.51-7.48 (m, 2H), $7.45(\mathrm{~d}, J=8.0 \mathrm{~Hz}, 1 \mathrm{H}), 7.42-7.40(\mathrm{~m}, 2 \mathrm{H}), 7.30-7.28(\mathrm{~m}$, 2H), 7.25-7.22 (m, 1H), $5.22(\mathrm{~s}, 2 \mathrm{H}), 3.61(\mathrm{~d}, J=19.5 \mathrm{~Hz}, 1 \mathrm{H}), 3.11(\mathrm{~d}, J=19.5 \mathrm{~Hz}$, 1H), 2.47 (s, 3H), 1.49 (s, 3H); ${ }^{13} \mathbf{C}$ NMR (125 MHz, $\left.\mathrm{CDCl}_{3}\right) \delta=200.9,175.5,163.5$, 138.7 (2C), 138.5, 137.8, 137.6, 137.0, 136.8, 133.2, 129.9, 129.7, 128.7 (2C), 128.5, 128.4, 127.5, 123.5, 56.7, 43.6, 43.5, 23.6, 20.9. HRMS (ESI-TOF) m/z: $[\mathrm{M}+\mathrm{H}]^{+}$ Calcd for $\mathrm{C}_{26} \mathrm{H}_{22} \mathrm{NO}_{3}{ }^{+}$396.1594; found 396.1596.<smiles>COc1cc2c3c(c1)-c1ccccc1C(=O)CC3(C)C(=O)N(Cc1ccccc1)C2=O</smiles>

\section{5-benzyl-2-methoxy-6a-methyl-6a,7-dihydrobenzo[4,5]cyclohepta[1,2,3-de]isoqui} noline-4,6,8(5H)-trione (3la): white solid, isolated yield 85\% (69.87 mg) (eluent: petroleum ether/EtOAc $=5: 1) ; \mathrm{mp}: 177.8-180.6{ }^{\circ} \mathrm{C}$ (uncorrected); ${ }^{1} \mathbf{H}$ NMR (500 $\left.\mathrm{MHz}, \mathrm{CDCl}_{3}\right) \delta=7.85(\mathrm{~s}, 1 \mathrm{H}), 7.74(\mathrm{~d}, J=7.5 \mathrm{~Hz}, 1 \mathrm{H}), 7.66(\mathrm{t}, J=7.0 \mathrm{~Hz}, 1 \mathrm{H}), 7.51$ (t, $J=7.5 \mathrm{~Hz}, 1 \mathrm{H}), 7.43(\mathrm{dd}, J=17.5,7.5 \mathrm{~Hz}, 3 \mathrm{H}), 7.31-7.25(\mathrm{~m}, 4 \mathrm{H}), 5.22(\mathrm{~s}, 2 \mathrm{H})$, $3.90(\mathrm{~s}, 3 \mathrm{H}), 3.60(\mathrm{~d}, J=19.0 \mathrm{~Hz}, 1 \mathrm{H}), 3.11(\mathrm{~d}, J=19.5 \mathrm{~Hz}, 1 \mathrm{H}), 1.48(\mathrm{~s}, 3 \mathrm{H}) ;{ }^{13} \mathrm{C}$ NMR $\left(125 \mathrm{MHz}, \mathrm{CDCl}_{3}\right) \delta=201.0,175.6,163.4,159.2,139.5,138.2,137.5,136.7$, $133.3,132.3,129.8,128.9,128.6,128.5,127.5,125.5,124.9,111.5,56.6,55.7,43.8$, 43.3, 23.8. HRMS (ESI-TOF) $\mathrm{m} / \mathrm{z}$ : $[\mathrm{M}+\mathrm{H}]^{+}$Calcd for $\mathrm{C}_{26} \mathrm{H}_{22} \mathrm{NO}_{4}{ }^{+}$412.1543; found 412.1546 . 
<smiles>CC12CC(=O)c3ccccc3-c3cc(F)cc(c31)C(=O)N2Cc1ccccc1</smiles>

5-benzyl-2-fluoro-6a-methyl-6a,7-dihydrobenzo[4,5]cyclohepta[1,2,3-de]isoquino line-4,6,8(5 $\mathrm{H}$ )-trione (3ma): white solid, isolated yield 60\% (47.88 mg) (eluent: petroleum ether/EtOAc $=10: 1) ; \mathrm{mp}: 175.2-177.3{ }^{\circ} \mathrm{C}$ (uncorrected); ${ }^{1} \mathbf{H}$ NMR (500 $\left.\mathrm{MHz}, \mathrm{CDCl}_{3}\right) \delta=8.04(\mathrm{dd}, J=8.0,2.5 \mathrm{~Hz}, 1 \mathrm{H}), 7.77(\mathrm{~d}, J=7.5 \mathrm{~Hz}, 1 \mathrm{H}), 7.70(\mathrm{t}, J=$ $7.5 \mathrm{~Hz}, 1 \mathrm{H}), 7.55$ (t, $J=7.5 \mathrm{~Hz}, 1 \mathrm{H}), 7.45-7.40$ (m, 4H), 7.30 (t, $J=7.0 \mathrm{~Hz}, 2 \mathrm{H})$, 7.26-7.23 (m, 1H), $5.21(\mathrm{~s}, 2 \mathrm{H}), 3.63$ (d, $J=19.5 \mathrm{~Hz}, 1 \mathrm{H}), 3.12$ (d, $J=19.5 \mathrm{~Hz}, 1 \mathrm{H})$, 1.49 (s, 3H); ${ }^{13} \mathrm{C}$ NMR (125 MHz, $\left.\mathrm{CDCl}_{3}\right) \delta=200.2,175.1,162.4,161.9$ (d, C-F, $\left.{ }^{1} J_{\mathrm{C}-\mathrm{F}}=247.5 \mathrm{~Hz}\right), 140.6\left(\mathrm{~d}, \mathrm{C}-\mathrm{F},{ }^{3} J_{\mathrm{C}-\mathrm{F}}=7.1 \mathrm{~Hz}\right), 137.3(2 \mathrm{C}), 136.4,135.8(\mathrm{~d}, \mathrm{C}-\mathrm{F}$, $\left.{ }^{4} J_{\mathrm{C}-\mathrm{F}}=3.3 \mathrm{~Hz}\right), 133.5,129.8,129.4,128.9,128.6,128.5,127.6,125.9\left(\mathrm{~d}, \mathrm{C}-\mathrm{F},{ }^{3} J_{\mathrm{C}-\mathrm{F}}=\right.$ $7.9 \mathrm{~Hz}), 124.5\left(\mathrm{~d}, \mathrm{C}-\mathrm{F},{ }^{2} J_{\mathrm{C}-\mathrm{F}}=22.8 \mathrm{~Hz}\right), 115.4\left(\mathrm{~d}, \mathrm{C}-\mathrm{F},{ }^{2} J_{\mathrm{C}-\mathrm{F}}=23.0 \mathrm{~Hz}\right), 56.4,43.9$, 43.5, 23.6. HRMS (ESI-TOF) m/z: $[\mathrm{M}+\mathrm{H}]^{+}$Calcd for $\mathrm{C}_{25} \mathrm{H}_{19} \mathrm{FNO}_{3}{ }^{+}$400.1343; found 400.1345 .<smiles>CC12CC(=O)c3ccccc3-c3cc(Cl)cc(c31)C(=O)N2Cc1ccccc1</smiles>

5-benzyl-2-chloro-6a-methyl-6a,7-dihydrobenzo[4,5]cyclohepta[1,2,3-de]isoquino line-4,6,8(5H)-trione (3na): white solid, isolated yield 56\% (46.48 mg) (eluent: petroleum ether/EtOAc $=10: 1) ; \mathrm{mp}: 212.3-213.5{ }^{\circ} \mathrm{C}$ (uncorrected); ${ }^{1} \mathbf{H}$ NMR $(500$ $\left.\mathrm{MHz}, \mathrm{CDCl}_{3}\right) \delta=8.35(\mathrm{~s}, 1 \mathrm{H}), 7.77(\mathrm{~d}, J=7.5 \mathrm{~Hz}, 1 \mathrm{H}), 7.68(\mathrm{~d}, J=13.0 \mathrm{~Hz}, 2 \mathrm{H})$, $7.55(\mathrm{t}, J=7.0 \mathrm{~Hz}, 1 \mathrm{H}), 7.43(\mathrm{dd}, J=22.5,7.0 \mathrm{~Hz}, 3 \mathrm{H}), 7.29(\mathrm{~d}, J=6.5 \mathrm{~Hz}, 2 \mathrm{H}), 7.26$ (s, 1H), $5.21(\mathrm{~s}, 2 \mathrm{H}), 3.63(\mathrm{~d}, J=19.0 \mathrm{~Hz}, 1 \mathrm{H}), 3.12$ (d, $J=19.5 \mathrm{~Hz}, 1 \mathrm{H}), 1.49$ (s, $3 \mathrm{H}) ;{ }^{13} \mathbf{C ~ N M R}\left(125 \mathrm{MHz}, \mathrm{CDCl}_{3}\right) \delta=200.1,175.0,162.3,139.9,138.2,137.4$, 137.2 , 137.0, 136.4, 134.9, 133.6, 129.9, 129.4, 129.0 (2C), 128.6, 128.5, 127.7, 125.3, 56.4, 43.9, 43.6, 23.4. HRMS (ESI-TOF) $\mathrm{m} / \mathrm{z}:[\mathrm{M}+\mathrm{H}]^{+}$Calcd for $\mathrm{C}_{25} \mathrm{H}_{19} \mathrm{ClNO}_{3}{ }^{+} \quad$ 416.1048; found 416.1050. 


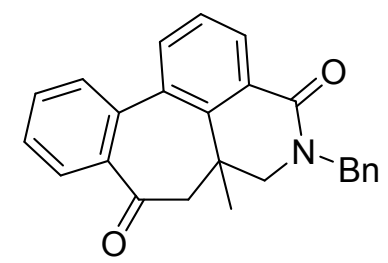

5-benzyl-6a-methyl-5,6,6a,7-tetrahydrobenzo[4,5]cyclohepta[1,2,3-de]isoquinolin e-4,8-dione (3pa): white solid, isolated yield 52\% (38.17 mg) (eluent: petroleum ether/EtOAc = 3:1); mp: 184.2-186.0 ${ }^{\circ} \mathrm{C}$ (uncorrected); ${ }^{1} \mathbf{H} \mathbf{N M R}\left(500 \mathrm{MHz}, \mathrm{CDCl}_{3}\right)$ $\delta=8.25(\mathrm{~d}, J=7.5 \mathrm{~Hz}, 1 \mathrm{H}), 7.98(\mathrm{~d}, J=7.5 \mathrm{~Hz}, 1 \mathrm{H}), 7.68(\mathrm{~d}, J=7.5 \mathrm{~Hz}, 1 \mathrm{H}), 7.63(\mathrm{t}$, $J=7.5 \mathrm{~Hz}, 1 \mathrm{H}), 7.51(\mathrm{t}, J=7.5 \mathrm{~Hz}, 1 \mathrm{H}), 7.46-7.42(\mathrm{~m}, 2 \mathrm{H}), 7.37-7.32(\mathrm{~m}, 4 \mathrm{H}), 7.29$ $(\mathrm{d}, J=6.5 \mathrm{~Hz}, 1 \mathrm{H}), 4.83(\mathrm{~d}, J=14.5 \mathrm{~Hz}, 1 \mathrm{H}), 4.71(\mathrm{~d}, J=14.5 \mathrm{~Hz}, 1 \mathrm{H}), 3.33$ (d, $J=$ $12.5 \mathrm{~Hz}, 1 \mathrm{H}), 3.12-3.05(\mathrm{~m}, 2 \mathrm{H}), 2.65$ (d, $J=17.5 \mathrm{~Hz}, 1 \mathrm{H}), 1.03(\mathrm{~s}, 3 \mathrm{H}) ;{ }^{13} \mathbf{C}$ NMR $\left(125 \mathrm{MHz}, \mathrm{CDCl}_{3}\right) \delta=199.7,164.5,143.1,139.5,137.4,136.3,135.5,135.1,133.5$, 131.9, 129.1, 128.9, 128.7, 128.6, 127.9, 127.8 (2C), 57.3, 55.8, 50.4, 35.1, 20.0. HRMS (ESI-TOF) m/z: $[\mathrm{M}+\mathrm{H}]^{+}$Calcd for $\mathrm{C}_{25} \mathrm{H}_{22} \mathrm{NO}_{2}{ }^{+}$368.1645; found 368.1647.<smiles>Cc1ccc2c(c1)-c1cccc3c1C(C)(CC(=O)N(Cc1ccccc1)C3=O)C2=O</smiles>

5-benzyl-6a,11-dimethyl-6a,7-dihydrobenzo[4,5]cyclohepta[1,2,3-de]isoquinoline4,6,8(5H)-trione (3ab): white solid, isolated yield 77\% (60.83 mg) (eluent: petroleum ether/EtOAc = 10:1); mp: $176.9-177.6^{\circ} \mathrm{C}$ (uncorrected); ${ }^{1} \mathbf{H} \mathbf{~ N M R}\left(500 \mathrm{MHz}, \mathrm{CDCl}_{3}\right)$ $\delta=8.38(\mathrm{~d}, J=8.0 \mathrm{~Hz}, 1 \mathrm{H}), 7.71(\mathrm{dd}, J=7.0,3.0 \mathrm{~Hz}, 2 \mathrm{H}), 7.59(\mathrm{t}, J=8.0 \mathrm{~Hz}, 1 \mathrm{H})$, $7.42(\mathrm{~d}, J=7.5 \mathrm{~Hz}, 2 \mathrm{H}), 7.33-7.28(\mathrm{~m}, 3 \mathrm{H}), 7.25$ (d, $J=6.5 \mathrm{~Hz}, 2 \mathrm{H}), 5.22(\mathrm{~s}, 2 \mathrm{H})$, $3.63(\mathrm{~d}, J=19.5 \mathrm{~Hz}, 1 \mathrm{H}), 3.10(\mathrm{~d}, J=19.0 \mathrm{~Hz}, 1 \mathrm{H}), 2.48(\mathrm{~s}, 3 \mathrm{H}), 1.52(\mathrm{~s}, 3 \mathrm{H}) ;{ }^{13} \mathrm{C}$ NMR $\left(125 \mathrm{MHz}, \mathrm{CDCl}_{3}\right) \delta=200.2,175.5,163.4,144.2,140.0,138.7,138.1,137.6$, $136.7,134.8,130.8,129.6,129.5,129.0,128.6,128.5$ (2C), 127.5, 123.5, 56.2, 43.9, 43.7, 23.4, 21.7. HRMS (ESI-TOF) m/z: $[\mathrm{M}+\mathrm{H}]^{+}$Calcd for $\mathrm{C}_{26} \mathrm{H}_{22} \mathrm{NO}_{3}{ }^{+}$396.1594; found 396.1596. 
<smiles>COc1ccc2c(c1)-c1cccc3c1C(C)(CC(=O)N(Cc1ccccc1)C3=O)C2=O</smiles>

5-benzyl-11-methoxy-6a-methyl-6a,7-dihydrobenzo[4,5]cyclohepta[1,2,3-de]isoqu inoline-4,6,8(5H)-trione (3ac): white solid, isolated yield 81\% (66.58 mg) (eluent: petroleum ether/EtOAc = 5:1); mp: 168.6-169.4 ${ }^{\circ} \mathrm{C}$ (uncorrected); ${ }^{1}$ H NMR (500 $\left.\mathrm{MHz}, \mathrm{CDCl}_{3}\right) \delta=8.39(\mathrm{~d}, J=8.0 \mathrm{~Hz}, 1 \mathrm{H}), 7.84(\mathrm{~d}, J=8.5 \mathrm{~Hz}, 1 \mathrm{H}), 7.72(\mathrm{~d}, J=7.5$ Hz, 1H), 7.59 (t, $J=8.0 \mathrm{~Hz}, 1 \mathrm{H}), 7.42$ (d, $J=7.0 \mathrm{~Hz}, 2 \mathrm{H}), 7.31-7.23(\mathrm{~m}, 3 \mathrm{H}), 7.01$ $(\mathrm{dd}, J=9.0,2.5 \mathrm{~Hz}, 1 \mathrm{H}), 6.93(\mathrm{~d}, J=2.5 \mathrm{~Hz}, 1 \mathrm{H}), 5.23(\mathrm{~s}, 2 \mathrm{H}), 3.92(\mathrm{~s}, 3 \mathrm{H}), 3.63(\mathrm{~d}$, $J=19.5 \mathrm{~Hz}, 1 \mathrm{H}), 3.07$ (d, $J=19.5 \mathrm{~Hz}, 1 \mathrm{H}), 1.54(\mathrm{~s}, 3 \mathrm{H}) ;{ }^{13} \mathbf{C} \mathbf{N M R}(125 \mathrm{MHz}$, $\left.\mathrm{CDCl}_{3}\right) \delta=198.7,175.5,163.5,163.4,141.0,140.2,137.9,137.6,136.7,131.6$, 130.1 , 129.7, 128.6, 128.5 (2C), 127.5, 123.5, 115.9, 113.5, 55.7, 55.6, 43.9, 43.7, 23.3. HRMS (ESI-TOF) m/z: $[\mathrm{M}+\mathrm{H}]^{+}$Calcd for $\mathrm{C}_{26} \mathrm{H}_{22} \mathrm{NO}_{4}{ }^{+} 412.1543$; found 412.1546.<smiles>CC12CC(=O)c3ccc(F)cc3-c3cccc(c31)C(=O)N2Cc1ccccc1</smiles>

5-benzyl-11-fluoro-6a-methyl-6a,7-dihydrobenzo[4,5]cyclohepta[1,2,3-de]isoquin oline-4,6,8(5H)-trione (3ad): white solid, isolated yield 75\% (59.85 mg) (eluent: petroleum ether/EtOAc $=10: 1) ; \mathrm{mp}: 174.8-177.1{ }^{\circ} \mathrm{C}$ (uncorrected); ${ }^{1} \mathbf{H}$ NMR (500 $\left.\mathrm{MHz}, \mathrm{CDCl}_{3}\right) \delta=8.43-8.41(\mathrm{~m}, 1 \mathrm{H}), 7.84(\mathrm{dd}, J=8.5,6.0 \mathrm{~Hz}, 1 \mathrm{H}), 7.71-7.69(\mathrm{~m}$, 1H), 7.62 (t, $J=8.0 \mathrm{~Hz}, 1 \mathrm{H}), 7.42$ (d, $J=7.5 \mathrm{~Hz}, 2 \mathrm{H}), 7.31$ (t, $J=7.0 \mathrm{~Hz}, 2 \mathrm{H})$, 7.27-7.25 (m, 1H), 7.23-7.16 (m, 2H), 5.23 (s, 2H), 3.66 (d, J=19.5 Hz, 1H), 3.12 (d, $J=19.5 \mathrm{~Hz}, 1 \mathrm{H}), 1.54(\mathrm{~s}, 3 \mathrm{H}) ;{ }^{13} \mathbf{C} \mathbf{N M R}\left(125 \mathrm{MHz}, \mathrm{CDCl}_{3}\right) \delta=198.9,175.2,165.5$ $\left(\mathrm{d}, \mathrm{C}-\mathrm{F},{ }^{1} J_{\mathrm{C}-\mathrm{F}}=251.5 \mathrm{~Hz}\right), 163.2,140.1,137.6,136.8,136.6,133.7\left(\mathrm{~d}, \mathrm{C}-\mathrm{F},{ }^{4} J_{\mathrm{C}-\mathrm{F}}=3.1\right.$ $\mathrm{Hz}), 131.9\left(\mathrm{~d}, \mathrm{C}-\mathrm{F},{ }^{3} J_{\mathrm{C}-\mathrm{F}}=9.5 \mathrm{~Hz}\right), 130.2,128.9,128.6,128.5,127.7,123.8,117.0(\mathrm{~d}$, $\left.\mathrm{C}-\mathrm{F},{ }^{2} J_{\mathrm{C}-\mathrm{F}}=22.9 \mathrm{~Hz}\right), 116.0\left(\mathrm{~d}, \mathrm{C}-\mathrm{F},{ }^{2} J_{\mathrm{C}-\mathrm{F}}=21.1 \mathrm{~Hz}\right), 56.1,43.8,23.6$. HRMS (ESI-TOF) m/z: $[\mathrm{M}+\mathrm{H}]^{+}$Calcd for $\mathrm{C}_{25} \mathrm{H}_{19} \mathrm{FNO}_{3}{ }^{+}$400.1343; found 400.1345. 
<smiles>CC12CC(=O)c3ccc(Cl)cc3-c3cccc(c31)C(=O)N(Cc1ccccc1)C2=O</smiles>

5-benzyl-11-chloro-6a-methyl-6a,7-dihydrobenzo[4,5]cyclohepta[1,2,3-de]isoquin oline-4,6,8(5H)-trione (3ae): white solid, isolated yield 77\% (63.91 mg) (eluent: petroleum ether/EtOAc $=10: 1) ; \mathrm{mp}: 177.8-180.0{ }^{\circ} \mathrm{C}$ (uncorrected); ${ }^{1} \mathbf{H}$ NMR $(500$ $\left.\mathrm{MHz}, \mathrm{CDCl}_{3}\right) \delta=8.42(\mathrm{~d}, J=7.5 \mathrm{~Hz}, 1 \mathrm{H}), 7.75(\mathrm{~d}, J=8.0 \mathrm{~Hz}, 1 \mathrm{H}), 7.70(\mathrm{~d}, J=7.5$ $\mathrm{Hz}, 1 \mathrm{H}), 7.62$ (t, $J=7.5 \mathrm{~Hz}, 1 \mathrm{H}), 7.50-7.47(\mathrm{~m}, 2 \mathrm{H}), 7.42$ (d, $J=7.5 \mathrm{~Hz}, 2 \mathrm{H}), 7.31$ (t, $J=7.5 \mathrm{~Hz}, 2 \mathrm{H}), 7.27-7.24(\mathrm{~m}, 1 \mathrm{H}), 5.22(\mathrm{~s}, 2 \mathrm{H}), 3.65(\mathrm{~d}, J=19.5 \mathrm{~Hz}, 1 \mathrm{H}), 3.12(\mathrm{~d}, J$ $=19.0 \mathrm{~Hz}, 1 \mathrm{H}), 1.54(\mathrm{~s}, 3 \mathrm{H}) ;{ }^{13} \mathbf{C} \mathbf{N M R}\left(125 \mathrm{MHz}, \mathrm{CDCl}_{3}\right) \delta=199.3,175.1,163.2$, $140.3,140.1,139.7,137.5,136.6,135.6,130.5,130.2,129.9,129.0,128.9,128.6$, 128.5, 127.6, 123.8, 56.2, 43.8, 43.7, 23.7. HRMS (ESI-TOF) m/z: $[\mathrm{M}+\mathrm{H}]^{+}$Calcd for $\mathrm{C}_{25} \mathrm{H}_{19} \mathrm{ClNO}_{3}{ }^{+} 416.1048$; found 416.1050.<smiles>CC12CC(=O)c3ccc(Oc4ccccc4)cc3-c3cccc(c31)C(=O)N(Cc1ccccc1)C2=O</smiles>

5-benzyl-6a-methyl-11-phenoxy-6a,7-dihydrobenzo[4,5]cyclohepta[1,2,3-de]isoqu inoline-4,6,8(5H)-trione (3af): white solid, isolated yield 75\% (70.95 mg) (eluent: petroleum ether/EtOAc $=5: 1) ; \mathrm{mp}: 225.6-226.8{ }^{\circ} \mathrm{C}$ (uncorrected); ${ }^{1} \mathbf{H}$ NMR (500 $\left.\mathrm{MHz}, \mathrm{CDCl}_{3}\right) \delta=8.38(\mathrm{dd}, J=7.5,1.0 \mathrm{~Hz}, 1 \mathrm{H}), 7.81(\mathrm{~d}, J=8.5 \mathrm{~Hz}, 1 \mathrm{H}), 7.66-7.65$ (m, 1H), 7.57 (t, $J=7.5 \mathrm{~Hz}, 1 \mathrm{H}), 7.43$ (t, $J=8.0 \mathrm{~Hz}, 4 \mathrm{H}), 7.31$ (t, $J=7.0 \mathrm{~Hz}, 2 \mathrm{H})$, 7.26-7.22 (m, 2H), $7.12(\mathrm{~d}, J=8.0 \mathrm{~Hz}, 2 \mathrm{H}), 7.06-7.02(\mathrm{~m}, 2 \mathrm{H}), 5.23(\mathrm{~s}, 2 \mathrm{H}), 3.65(\mathrm{~d}$, $J=19.5 \mathrm{~Hz}, 1 \mathrm{H}), 3.09$ (d, $J=19.5 \mathrm{~Hz}, 1 \mathrm{H}), 1.58(\mathrm{~s}, 3 \mathrm{H}) ;{ }^{13} \mathbf{C}$ NMR (125 MHz, $\left.\mathrm{CDCl}_{3}\right) \delta=198.9,175.4,163.4,162.4,155.1,141.2,140.2,137.6,137.5,136.7$, $131.8,131.6,130.2$, 129.9, 128.8, 128.6, 128.5, 127.6, 124.9, 123.6, 120.2, 118.8, 117.2, 56.0, 43.9, 43.8, 23.5. HRMS (ESI-TOF) m/z: $[\mathrm{M}+\mathrm{H}]^{+}$Calcd for $\mathrm{C}_{31} \mathrm{H}_{24} \mathrm{NO}_{4}{ }^{+}$ 474.1700; found 474.1702. 
(l)

5-benzyl-12-methoxy-6a-methyl-6a,7-dihydrobenzo[4,5]cyclohepta[1,2,3-de]isoqu inoline-4,6,8(5H)-trione (3ag): white solid, isolated yield 61\% (50.14 mg) (eluent: petroleum ether/EtOAc $=5: 1) ; \mathrm{mp}: 131.8-132.9{ }^{\circ} \mathrm{C}$ (uncorrected); ${ }^{1} \mathbf{H}$ NMR $(500$ $\left.\mathrm{MHz}, \mathrm{CDCl}_{3}\right) \delta=8.34(\mathrm{~d}, J=7.0 \mathrm{~Hz}, 1 \mathrm{H}), 7.76(\mathrm{~d}, J=7.0 \mathrm{~Hz}, 1 \mathrm{H}), 7.52-7.50(\mathrm{~m}$, 1H), 7.47-7.44 (m, 1H), 7.40 (d, $J=6.0 \mathrm{~Hz}, 2 \mathrm{H}), 7.29$ (d, $J=7.0 \mathrm{~Hz}, 2 \mathrm{H}), 7.24-7.19$ (m, 3H), $5.21(\mathrm{~s}, 2 \mathrm{H}), 3.81(\mathrm{~s}, 3 \mathrm{H}), 3.46(\mathrm{~d}, J=19.0 \mathrm{~Hz}, 1 \mathrm{H}), 3.07$ (d, $J=19.0 \mathrm{~Hz}$, 1H), $1.56(\mathrm{~s}, 3 \mathrm{H}) ;{ }^{13} \mathbf{C} \mathbf{N M R}\left(125 \mathrm{MHz}, \mathrm{CDCl}_{3}\right) \delta=201.7,175.4,163.3,155.2,139.8$, 139.6, 136.7, 132.5, 130.0, 129.3, 128.6, 128.4 (2C), 127.4 (2C), 126.5, 123.7, 119.8, 115.4, 57.9, 55.9, 43.8, 43.5, 23.1. HRMS (ESI-TOF) m/z: $[\mathrm{M}+\mathrm{H}]^{+}$Calcd for $\mathrm{C}_{26} \mathrm{H}_{22} \mathrm{NO}_{4}{ }^{+}$412.1543; found 412.1546 .<smiles>COc1ccc2c(c1)C(=O)CC1(C)C(=O)N(Cc3ccccc3)C(=O)c3cccc-2c31</smiles>

5-benzyl-10-methoxy-6a-methyl-6a,7-dihydrobenzo[4,5]cyclohepta[1,2,3-de]isoqu inoline-4,6,8(5H)-trione (3ah): white solid, isolated yield 69\% (56.72 mg) (eluent: petroleum ether/EtOAc $=5: 1)$; mp: 132.6-133.7 ${ }^{\circ} \mathrm{C}$ (uncorrected); ${ }^{1} \mathbf{H}$ NMR (500 MHz, DMSO-d 6 ) $\delta=8.23(\mathrm{~d}, J=8.0 \mathrm{~Hz}, 1 \mathrm{H}), 7.82(\mathrm{~d}, J=7.5 \mathrm{~Hz}, 1 \mathrm{H}), 7.69(\mathrm{t}, J=$ $8.0 \mathrm{~Hz}, 1 \mathrm{H}), 7.53$ (d, $J=8.5 \mathrm{~Hz}, 1 \mathrm{H}), 7.35$ (d, $J=8.5 \mathrm{~Hz}, 1 \mathrm{H}), 7.32-7.29$ (m, 4H), $7.24(\mathrm{~d}, J=6.5 \mathrm{~Hz}, 1 \mathrm{H}), 7.19$ (s, 1H), 5.10 (s, 2H), 3.87 (s, 3H), 3.43 (s, 2H), 1.42 (s, $3 \mathrm{H}) .{ }^{13} \mathrm{C}$ NMR (125 MHz, DMSO-d $\left.{ }_{6}\right) \delta=200.4,175.3,163.2,159.3,139.5,138.1$, $137.3,137.2$, 137.0, 132.2, 130.5, 128.9, 128.4, 128.3, 127.1 (2C), 123.6, 119.6, 112.6, 55.7, 55.6, 43.2 (2C), 23.0. HRMS (ESI-TOF) m/z: $[\mathrm{M}+\mathrm{H}]^{+}$Calcd for $\mathrm{C}_{26} \mathrm{H}_{22} \mathrm{NO}_{4}{ }^{+}$412.1543; found 412.1546 . 
<smiles>CC12CC(=O)c3ccc(N4CCOCC4)cc3-c3cccc(c31)C(=O)N(Cc1ccccc1)C2=O</smiles>

5-benzyl-6a-methyl-11-morpholino-6a,7-dihydrobenzo[4,5]cyclohepta[1,2,3-de]is oquinoline-4,6,8(5H)-trione (3ai): white solid, isolated yield 87\% (81.08 $\mathrm{mg}$ ) (eluent: petroleum ether/EtOAc = 3:1); mp: 211.5-212.6 ${ }^{\circ} \mathrm{C}$ (uncorrected); ${ }^{1} \mathbf{H}$ NMR $\left(500 \mathrm{MHz}, \mathrm{CDCl}_{3}\right) \delta=8.37(\mathrm{~d}, J=8.0 \mathrm{~Hz}, 1 \mathrm{H}), 7.82(\mathrm{~d}, J=8.5 \mathrm{~Hz}, 1 \mathrm{H}), 7.72(\mathrm{~d}, J=$ $7.5 \mathrm{~Hz}, 1 \mathrm{H}), 7.57$ (t, $J=8.0 \mathrm{~Hz}, 1 \mathrm{H}), 7.42$ (d, $J=7.5 \mathrm{~Hz}, 2 \mathrm{H}), 7.31-7.22(\mathrm{~m}, 3 \mathrm{H})$, $6.95(\mathrm{dd}, J=9.0,1.5 \mathrm{~Hz}, 1 \mathrm{H}), 6.82(\mathrm{~s}, 1 \mathrm{H}), 5.22(\mathrm{~s}, 2 \mathrm{H}), 3.87$ (t, $J=4.0 \mathrm{~Hz}, 4 \mathrm{H}), 3.61$ $(\mathrm{d}, J=19.0 \mathrm{~Hz}, 1 \mathrm{H}), 3.42-3.32(\mathrm{~m}, 4 \mathrm{H}), 3.03(\mathrm{~d}, J=19.0 \mathrm{~Hz}, 1 \mathrm{H}), 1.55(\mathrm{~s}, 3 \mathrm{H}) ;{ }^{13} \mathrm{C}$ NMR $\left(125 \mathrm{MHz}, \mathrm{CDCl}_{3}\right) \delta=197.9,175.5,163.4,154.3,140.8,140.4,138.7,137.4$, 136.7, 131.2, 129.5, 128.5, 128.4, 127.5 (2C), 123.3, 114.9, 113.6, 66.4, 55.4, 47.3, 43.9, 43.6, 23.1. HRMS (ESI-TOF) $\mathrm{m} / \mathrm{z}$ : $[\mathrm{M}+\mathrm{H}]^{+}$Calcd for $\mathrm{C}_{29} \mathrm{H}_{27} \mathrm{~N}_{2} \mathrm{O}_{4}{ }^{+}$467.1965; found 467.1968 .<smiles>COc1cc2c(cc1OC)-c1cccc3c1C(C)(CC(=O)C3=O)C(=O)N(Cc1ccccc1)C2=O</smiles>

5-benzyl-10,11-dimethoxy-6a-methyl-6a,7-dihydrobenzo[4,5]cyclohepta[1,2,3-de] isoquinoline-4,6,8(5H)-trione (3aj): white solid, isolated yield 76\% (67.03 $\mathrm{mg})$ (eluent: petroleum ether/EtOAc $=4: 1$ ); mp: 195.7-197.1 ${ }^{\circ} \mathrm{C}$ (uncorrected); ${ }^{1} \mathbf{H}$ NMR $\left(500 \mathrm{MHz}, \mathrm{CDCl}_{3}\right) \delta=8.36(\mathrm{~d}, J=7.0 \mathrm{~Hz}, 1 \mathrm{H}), 7.72(\mathrm{~d}, J=8.0 \mathrm{~Hz}, 1 \mathrm{H}), 7.59(\mathrm{t}, J=$ 7.5 Hz, 1H), 7.43-7.40 (m, 3H), 7.31-7.23 (m, 3H), 6.89 (s, 1H), $5.23(\mathrm{~s}, 2 \mathrm{H}), 4.01(\mathrm{~d}$, $J=5.5 \mathrm{~Hz}, 6 \mathrm{H}), 3.68(\mathrm{~d}, J=19.5 \mathrm{~Hz}, 1 \mathrm{H}), 3.07(\mathrm{~d}, J=19.5 \mathrm{~Hz}, 1 \mathrm{H}), 1.54(\mathrm{~s}, 3 \mathrm{H}) ;{ }^{13} \mathrm{C}$ NMR $\left(125 \mathrm{MHz}, \mathrm{CDCl}_{3}\right) \delta=198.5,175.5,163.4,153.0,149.1,140.1,137.9,137.3$, 136.6, 133.0, 129.6, 129.1, 128.5 (2C), 128.4, 127.5, 123.4, 112.6, 111.2, 56.2, 56.1, 55.5, 43.8, 43.7, 22.9. HRMS (ESI-TOF) $\mathrm{m} / \mathrm{z}$ : $[\mathrm{M}+\mathrm{H}]^{+}$Calcd for $\mathrm{C}_{27} \mathrm{H}_{24} \mathrm{NO}_{5}{ }^{+}$ 442.1649; found 442.1651. 
<smiles>CC12CC(=O)c3cc4c(cc3-c3cccc(c31)C(=O)N(Cc1ccccc1)C4=O)OCO2</smiles>

5-benzyl-6a-methyl-6a,7-dihydro-[1,3] dioxolo[4",5'":4',5']benzo[1',2':4,5]cyclohe pta[1,2,3-de]isoquinoline-4,6,8(5H)-trione (3ak): white solid, isolated yield 65\% (55.25 mg) (eluent: petroleum ether/EtOAc $=4: 1)$; mp: 208.7-210.6 ${ }^{\circ} \mathrm{C}$ (uncorrected); ${ }^{1} \mathbf{H}$ NMR $\left(500 \mathrm{MHz}, \mathrm{CDCl}_{3}\right) \delta=8.35(\mathrm{~d}, J=7.5 \mathrm{~Hz}, 1 \mathrm{H}), 7.64(\mathrm{~d}, J=7.5 \mathrm{~Hz}, 1 \mathrm{H})$, $7.57(\mathrm{t}, J=7.5 \mathrm{~Hz}, 1 \mathrm{H}), 7.42(\mathrm{~d}, J=7.0 \mathrm{~Hz}, 2 \mathrm{H}), 7.32-7.23(\mathrm{~m}, 4 \mathrm{H}), 6.88(\mathrm{~s}, 1 \mathrm{H})$, $6.12(\mathrm{~s}, 2 \mathrm{H}), 5.22(\mathrm{~s}, 2 \mathrm{H}), 3.63(\mathrm{~d}, J=19.0 \mathrm{~Hz}, 1 \mathrm{H}), 3.06$ (d, $J=19.5 \mathrm{~Hz}, 1 \mathrm{H}), 1.58$ (s, $3 \mathrm{H}) ;{ }^{13} \mathbf{C}$ NMR $\left(125 \mathrm{MHz}, \mathrm{CDCl}_{3}\right) \delta=198.7,175.4,163.4,152.1,148.2,139.9$, $137.7,137.5,136.7,134.9,131.8,129.3,128.7,128.6,128.5,127.6,123.5,110.0$, 108.6, 102.3, 55.9, 43.8, 43.7, 23.1. HRMS (ESI-TOF) m/z: $[\mathrm{M}+\mathrm{H}]^{+}$Calcd for $\mathrm{C}_{26} \mathrm{H}_{20} \mathrm{NO}_{5}^{+}$426.1336; found 426.1338.<smiles>CC12CC(=O)c3sccc3-c3cccc(c31)C(=O)N(Cc1ccccc1)C2=O</smiles>

8-benzyl-9a-methyl-9a,10-dihydrothieno[ $\left[3^{\prime}, 2^{\prime}: 4,5\right]$ cyclohepta[1,2,3-de]isoquinolin e-7,9,11(8H)-trione (3al): white solid, isolated yield 45\% (34.8 mg) (eluent: petroleum ether/EtOAc = 5:1); mp: 218.7-220.6 ${ }^{\circ} \mathrm{C}$ (uncorrected); ${ }^{1} \mathbf{H}$ NMR (500 $\left.\mathrm{MHz}, \mathrm{CDCl}_{3}\right) \delta=8.41-8.40(\mathrm{~m}, 1 \mathrm{H}), 7.99(\mathrm{~d}, J=7.5 \mathrm{~Hz}, 1 \mathrm{H}), 7.74(\mathrm{~d}, J=5.5 \mathrm{~Hz}$, $1 \mathrm{H}), 7.60$ (t, $J=7.5 \mathrm{~Hz}, 1 \mathrm{H}), 7.42(\mathrm{dd}, J=15.5,7.5 \mathrm{~Hz}, 3 \mathrm{H}), 7.30(\mathrm{t}, J=7.5 \mathrm{~Hz}, 2 \mathrm{H})$, 7.26-7.23 (m, 1H), $5.23(\mathrm{~s}, 2 \mathrm{H}), 3.88(\mathrm{~d}, J=17.0 \mathrm{~Hz}, 1 \mathrm{H}), 3.05(\mathrm{~d}, J=17.0 \mathrm{~Hz}, 1 \mathrm{H})$, $1.52(\mathrm{~s}, 3 \mathrm{H}) ;{ }^{13} \mathrm{C}$ NMR $\left(125 \mathrm{MHz}, \mathrm{CDCl}_{3}\right) \delta=189.6,175.2,163.3,142.5,140.8$, $139.8,136.6,136.2,133.7,132.4,130.5,130.2,128.8,128.5,128.4,127.6,124.4$, 50.7, 44.1, 43.8, 22.7. HRMS (ESI-TOF) $\mathrm{m} / \mathrm{z}$ : $[\mathrm{M}+\mathrm{H}]^{+}$Calcd for $\mathrm{C}_{23} \mathrm{H}_{18} \mathrm{NO}_{3} \mathrm{~S}^{+}$ 388.1002; found 388.0999. 


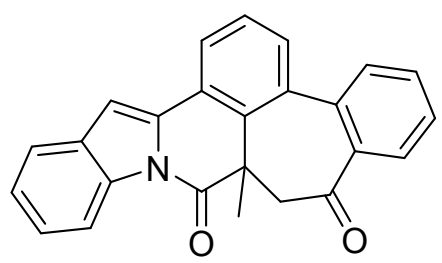

9a-methyl-9,9a-dihydrobenzo[4,5] cyclohepta[1,2,3-de]indolo[2,1-a]isoquinoline-8 ,10-dione (5aa): white solid, isolated yield 78\% (56.94 mg), 38\% (27.74 mg, 1-(2-(2-bromophenyl)-1H-indol-1-yl)-2-methylprop-2-en-1-one was used) (eluent: petroleum ether/EtOAc $=10: 1) ; \mathrm{mp}: 191.0-193.7{ }^{\circ} \mathrm{C}$ (uncorrected); ${ }^{1} \mathbf{H}$ NMR (500 $\left.\mathrm{MHz}, \mathrm{CDCl}_{3}\right) \delta=8.57(\mathrm{~d}, J=8.0 \mathrm{~Hz}, 1 \mathrm{H}), 7.96(\mathrm{~d}, J=8.0 \mathrm{~Hz}, 1 \mathrm{H}), 7.77-7.76(\mathrm{~m}$, 1H), 7.69-7.62 (m, 2H), 7.54-7.50 (m, 3H), 7.43-7.35 (m, 3H), $7.12(\mathrm{~s}, 1 \mathrm{H}), 3.77(\mathrm{~s}$, 1H), $3.75(\mathrm{~d}, J=19.5 \mathrm{~Hz}, 1 \mathrm{H}), 3.26(\mathrm{~d}, J=19.5 \mathrm{~Hz}, 1 \mathrm{H}), 1.62(\mathrm{~s}, 3 \mathrm{H}) ;{ }^{13} \mathbf{C}$ NMR $(125$ $\left.\mathrm{MHz}, \mathrm{CDCl}_{3}\right) \delta=201.5,172.1,139.6,138.7,137.6,135.1,134.9,134.8,133.2,132.8$, $130.7,129.9,128.6,128.5,128.4,125.4,124.9,124.0,123.4,120.5,116.9,103.9$, 56.5, 44.7, 23.4. HRMS (ESI-TOF) m/z: $[\mathrm{M}+\mathrm{H}]^{+}$Calcd for $\mathrm{C}_{25} \mathrm{H}_{18} \mathrm{NO}_{2}{ }^{+}$364.1332; found 364.1334 .

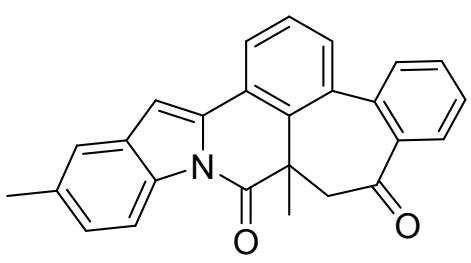

9a,14-dimethyl-9,9a-dihydrobenzo[4,5]cyclohepta[1,2,3-de $]$ indolo[2,1-a]isoquinol ine-8,10-dione (5ba): white solid, isolated yield 71\% (53.53 mg) (eluent: petroleum ether/EtOAc = 10:1); mp: $231.6-234.6^{\circ} \mathrm{C}$ (uncorrected); ${ }^{1} \mathbf{H} \mathbf{~ N M R}\left(500 \mathrm{MHz}, \mathrm{CDCl}_{3}\right)$ $\delta=8.42(\mathrm{~d}, J=8.5 \mathrm{~Hz}, 1 \mathrm{H}), 7.93(\mathrm{~d}, J=7.5 \mathrm{~Hz}, 1 \mathrm{H}), 7.75(\mathrm{~d}, J=8.0 \mathrm{~Hz}, 1 \mathrm{H}), 7.66(\mathrm{t}$, $J=7.5 \mathrm{~Hz}, 1 \mathrm{H}), 7.52-7.48(\mathrm{~m}, 3 \mathrm{H}), 7.40(\mathrm{~d}, J=7.0 \mathrm{~Hz}, 2 \mathrm{H}), 7.21(\mathrm{~d}, J=8.5 \mathrm{~Hz}, 1 \mathrm{H})$, 7.04 (s, 1H), 3.74 (d, $J=19.5 \mathrm{~Hz}, 1 \mathrm{H}), 3.25$ (d, $J=19.5 \mathrm{~Hz}, 1 \mathrm{H}), 2.47$ (s, 3H), 1.60 (s, $3 \mathrm{H}) ;{ }^{13} \mathbf{C}$ NMR $\left(125 \mathrm{MHz}, \mathrm{CDCl}_{3}\right) \delta=201.7,171.9,139.7,138.8,137.7,134.9$ (2C), $134.7,133.3,133.2,132.7,131.0,129.9,128.6$, 128.5, 128.4, 126.8, 124.0, 123.6, 120.5, 116.5, 103.7, 56.5, 44.7, 23.4, 21.5. HRMS (ESI-TOF) m/z: $[\mathrm{M}+\mathrm{H}]^{+}$Calcd for $\mathrm{C}_{26} \mathrm{H}_{19} \mathrm{NO}_{2}{ }^{+} 377.1416$; found 377.1413 . 


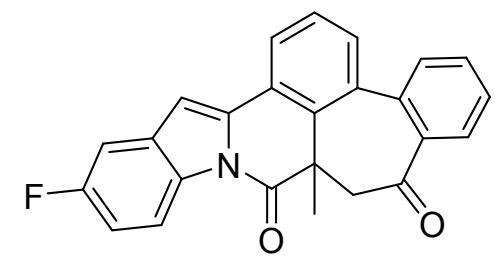

14-fluoro-9a-methyl-9,9a-dihydrobenzo[4,5]cyclohepta[1,2,3-de] indolo[2,1-a]isoq uinoline-8,10-dione (5ca): white solid, isolated yield 73\% (55.92 mg) (eluent: petroleum ether/EtOAc $=10: 1) ; \mathrm{mp}: 158.5-161.6{ }^{\circ} \mathrm{C}$ (uncorrected); ${ }^{1} \mathbf{H}$ NMR $(500$ $\left.\mathrm{MHz}, \mathrm{CDCl}_{3}\right) \delta=8.52(\mathrm{dd}, J=9.0,5.0 \mathrm{~Hz}, 1 \mathrm{H}), 7.93(\mathrm{~d}, J=7.5 \mathrm{~Hz}, 1 \mathrm{H}), 7.76(\mathrm{~d}, J=$ $7.5 \mathrm{~Hz}, 1 \mathrm{H}), 7.70-7.66(\mathrm{~m}, 1 \mathrm{H}), 7.54-7.49(\mathrm{~m}, 3 \mathrm{H}), 7.44(\mathrm{~d}, J=7.5 \mathrm{~Hz}, 1 \mathrm{H})$, 7.28-7.25 (m, 1H), 7.12-7.07 (m, 2H), 3.73 (d, $J=19.0 \mathrm{~Hz}, 1 \mathrm{H}), 3.25$ (d, $J=19.5 \mathrm{~Hz}$, 1H), $1.61(\mathrm{~s}, 3 \mathrm{H}) ;{ }^{13} \mathrm{C}$ NMR $\left(125 \mathrm{MHz}, \mathrm{CDCl}_{3}\right) \delta=201.4,171.9,160.4\left(\mathrm{~d}, \mathrm{C}-\mathrm{F},{ }^{1} J_{\mathrm{C}-\mathrm{F}}\right.$ $=240.4 \mathrm{~Hz}), 139.5,138.9,137.6,136.4,135.2,133.2(2 \mathrm{C}), 132.0\left(\mathrm{~d}, \mathrm{C}-\mathrm{F},{ }^{3} J_{\mathrm{C}-\mathrm{F}}=10.3\right.$ $\mathrm{Hz}), 131.5,130.0,128.7,128.6,128.5,124.2,123.1,118.0\left(\mathrm{~d}, \mathrm{C}-\mathrm{F},{ }^{3} J_{\mathrm{C}-\mathrm{F}}=9.0 \mathrm{~Hz}\right)$, $113.0\left(\mathrm{~d}, \mathrm{C}-\mathrm{F},{ }^{2} J_{\mathrm{C}-\mathrm{F}}=24.6 \mathrm{~Hz}\right), 106.2\left(\mathrm{~d}, \mathrm{C}-\mathrm{F},{ }^{2} J_{\mathrm{C}-\mathrm{F}}=23.9 \mathrm{~Hz}\right), 103.4\left(\mathrm{~d}, \mathrm{C}-\mathrm{F},{ }^{4} J_{\mathrm{C}-\mathrm{F}}=\right.$ 4.0 Hz), 56.4, 44.7, 23.4. HRMS (ESI-TOF) m/z: $[\mathrm{M}+\mathrm{H}]^{+}$Calcd for : $\mathrm{C}_{25} \mathrm{H}_{17} \mathrm{FNO}_{2}{ }^{+}$ 382.1238; found 382.1240 .

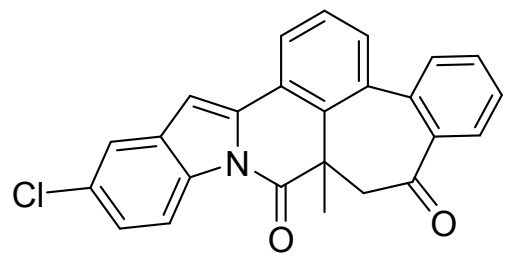

14-chloro-9a-methyl-9,9a-dihydrobenzo[4,5]cyclohepta[1,2,3-de]indolo[2,1-a]isoq uinoline-8,10-dione (5da): white solid, isolated yield 63\% (50.27 mg) (eluent: petroleum ether/EtOAc $=10: 1) ; \mathrm{mp}$ : 201.3-203.7 ${ }^{\circ} \mathrm{C}$ (uncorrected); ${ }^{1} \mathbf{H}$ NMR (500 $\left.\mathrm{MHz}, \mathrm{CDCl}_{3}\right) \delta=8.46(\mathrm{~d}, J=8.5 \mathrm{~Hz}, 1 \mathrm{H}), 7.91(\mathrm{~d}, J=8.0 \mathrm{~Hz}, 1 \mathrm{H}), 7.75(\mathrm{~d}, J=7.5$ $\mathrm{Hz}, 1 \mathrm{H}), 7.68-7.65$ (m, 1H), 7.55-7.41 (m, 5H), 7.31 (dd, $J=8.5,1.0 \mathrm{~Hz}, 1 \mathrm{H}), 7.01$ (s, 1H), $3.71(\mathrm{~d}, J=19.5 \mathrm{~Hz}, 1 \mathrm{H}), 3.23$ (d, $J=19.5 \mathrm{~Hz}, 1 \mathrm{H}), 1.59$ (s, 3H); ${ }^{13} \mathbf{C}$ NMR $(125$ $\left.\mathrm{MHz}, \mathrm{CDCl}_{3}\right) \delta=201.2,172.0,139.4,138.8,137.6,136.1,135.1,133.4,133.2,132.0$, $130.5,129.9,128.7,128.6,128.5,125.4,124.2,122.9,120.1,117.8,102.9,56.4,44.7$, 23.4. HRMS (ESI-TOF) m/z: $[\mathrm{M}+\mathrm{H}]^{+}$Calcd for $\mathrm{C}_{25} \mathrm{H}_{17} \mathrm{ClNO}_{2}{ }^{+}$398.0942; found 398.0944 . 
<smiles>CC12CC(=O)c3ccccc3-c3cccc(c31)-c1cc3cc(Br)ccc3n1C2=O</smiles>

14-bromo-9a-methyl-9,9a-dihydrobenzo[4,5]cyclohepta[1,2,3-de] indolo[2,1-a]iso quinoline-8,10-dione (5ea): white solid, isolated yield 54\% (47.84 mg) (eluent: petroleum ether/EtOAc $=10: 1) ; \mathrm{mp}: 217.4-222.8{ }^{\circ} \mathrm{C}$ (uncorrected); ${ }^{1} \mathbf{H}$ NMR $(500$ $\left.\mathrm{MHz}, \mathrm{CDCl}_{3}\right) \delta=8.42(\mathrm{~d}, J=9.0 \mathrm{~Hz}, 1 \mathrm{H}), 7.92(\mathrm{~d}, J=7.5 \mathrm{~Hz}, 1 \mathrm{H}), 7.77-7.73(\mathrm{~m}$, 2H), $7.68(\mathrm{t}, J=7.5 \mathrm{~Hz}, 1 \mathrm{H}), 7.54-7.43(\mathrm{~m}, 5 \mathrm{H}), 7.02(\mathrm{~s}, 1 \mathrm{H}), 3.72(\mathrm{~d}, J=19.0 \mathrm{~Hz}$, $1 \mathrm{H}), 3.24(\mathrm{~d}, J=19.5 \mathrm{~Hz}, 1 \mathrm{H}), 1.60(\mathrm{~s}, 3 \mathrm{H}) ;{ }^{13} \mathbf{C ~ N M R}\left(125 \mathrm{MHz}, \mathrm{CDCl}_{3}\right) \delta=201.3$, $172.1,139.4,138.9,137.6,136.0,135.1,133.8,133.3(2 \mathrm{C}), 132.5,123.0,128.7$, 128.6, 128.5, 128.2, 124.2, 123.2, 122.9, 118.3, 118.2, 102.8, 56.4, 44.8, 23.4. HRMS (ESI-TOF) m/z: [M + H] $]^{+}$Calcd for $\mathrm{C}_{25} \mathrm{H}_{17} \mathrm{BrNO}_{2}{ }^{+}$442.0437; found 442.0439.

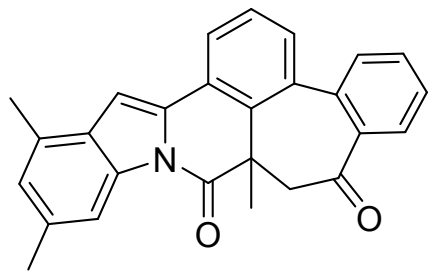

9a,13,15-trimethyl-9,9a-dihydrobenzo[4,5]cyclohepta[1,2,3-de]indolo[2,1-a] isoqui noline-8,10-dione (5fa): white solid, isolated yield 80\% (62.56 mg) (eluent: petroleum ether/EtOAc $=10: 1) ; \mathrm{mp}: 230.0-234.1{ }^{\circ} \mathrm{C}$ (uncorrected); ${ }^{1} \mathbf{H}$ NMR $(500$ $\left.\mathrm{MHz}, \mathrm{CDCl}_{3}\right) \delta=8.25(\mathrm{~s}, 1 \mathrm{H}), 7.95(\mathrm{~d}, J=8.0 \mathrm{~Hz}, 1 \mathrm{H}), 7.75(\mathrm{~d}, J=7.5 \mathrm{~Hz}, 1 \mathrm{H})$, 7.68-7.65 (m, 1H), $7.50(\mathrm{td}, J=8.0,3.5 \mathrm{~Hz}, 3 \mathrm{H}), 7.38(\mathrm{~d}, J=7.5 \mathrm{~Hz}, 1 \mathrm{H}), 7.11(\mathrm{~s}$, 1H), $7.00(\mathrm{~s}, 1 \mathrm{H}), 3.74(\mathrm{~d}, J=19.5 \mathrm{~Hz}, 1 \mathrm{H}), 3.25(\mathrm{~d}, J=19.0 \mathrm{~Hz}, 1 \mathrm{H}), 2.55(\mathrm{~s}, 3 \mathrm{H})$, $2.48(\mathrm{~s}, 3 \mathrm{H}), 1.60(\mathrm{~s}, 3 \mathrm{H}) ;{ }^{13} \mathrm{C}$ NMR $\left(125 \mathrm{MHz}, \mathrm{CDCl}_{3}\right) \delta=201.8,172.2,139.7$, $138.8,137.7,135.9,135.3,134.6,133.6,133.1,132.4,129.9,129.6,128.5(2 \mathrm{C})$, $128.4,128.0,126.9,123.8,123.8,114.6,102.5,56.56,44.8,23.5,21.9,18.4$. HRMS (ESI-TOF) $\mathrm{m} / \mathrm{z}$ : $[\mathrm{M}+\mathrm{H}]^{+}$Calcd for $\mathrm{C}_{27} \mathrm{H}_{21} \mathrm{NO}_{2}{ }^{+}$391.1572; found 391.1571. 


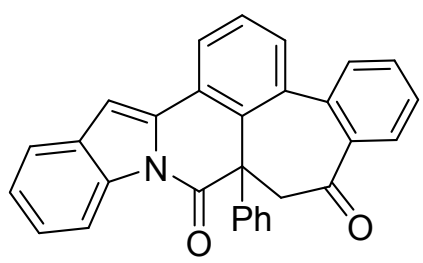

9a-phenyl-9,9a-dihydrobenzo[4,5] cyclohepta[1,2,3-de]indolo[2,1-a]isoquinoline-8 ,10-dione (5ga): white solid, isolated yield 63\% (53.80 mg) (eluent: petroleum ether/EtOAc = 10:1); mp: $237.7-240.8{ }^{\circ} \mathrm{C}$ (uncorrected); ${ }^{1} \mathbf{H}$ NMR $\left(500 \mathrm{MHz}, \mathrm{CDCl}_{3}\right)$ $\delta=8.43-8.41(\mathrm{~m}, 1 \mathrm{H}), 8.05(\mathrm{~d}, J=8.0 \mathrm{~Hz}, 1 \mathrm{H}), 7.63-7.55(\mathrm{~m}, 3 \mathrm{H}), 7.38(\mathrm{~d}, J=7.5 \mathrm{~Hz}$, 1H), $7.32(\mathrm{~d}, J=4.0 \mathrm{~Hz}, 2 \mathrm{H}), 7.30-7.25(\mathrm{~m}, 2 \mathrm{H}), 7.19(\mathrm{~d}, J=7.5 \mathrm{~Hz}, 2 \mathrm{H}), 7.01-6.94$ $(\mathrm{m}, 5 \mathrm{H}), 4.55(\mathrm{~d}, J=20.0 \mathrm{~Hz}, 1 \mathrm{H}), 3.46(\mathrm{~d}, J=20.0 \mathrm{~Hz}, 1 \mathrm{H}) ;{ }^{13} \mathrm{C}$ NMR $(125 \mathrm{MHz}$, $\left.\mathrm{CDCl}_{3}\right) \delta=201.1,169.2,139.7,139.1,138.8,137.5,135.6,135.4,134.8,132.7$, $132.5,130.8,123.0,128.9,128.0,127.9,127.8,127.7,127.0,125.5,125.0,124.4$, 123.7, 120.6, 117.0, 104.3, 54.5, 52.8. HRMS (ESI-TOF) m/z: $[\mathrm{M}+\mathrm{H}]^{+}$Calcd for $\mathrm{C}_{30} \mathrm{H}_{20} \mathrm{NO}_{2}{ }^{+}$426.1489; found 426.1491 .



9a-methyl-9a,10-dihydrobenzo[4,5] cyclohepta[1,2,3-de]indolo[2,1-a]isoquinolin-8 (9H)-one (5ha): white solid, isolated yield 48\% (33.70 mg) (eluent: petroleum ether/EtOAc = 3:1); mp: 209.6-210.3 ${ }^{\circ} \mathrm{C}$ (uncorrected); ${ }^{1} \mathbf{H} \mathbf{N M R}\left(500 \mathrm{MHz}, \mathrm{CDCl}_{3}\right)$ $\delta=7.98-7.96(\mathrm{~m}, 1 \mathrm{H}), 7.84(\mathrm{dd}, J=6.0,3.0 \mathrm{~Hz}, 1 \mathrm{H}), 7.65-7.62(\mathrm{~m}, 2 \mathrm{H}), 7.45-7.41(\mathrm{~m}$, 4H), $7.35(\mathrm{~d}, J=8.0 \mathrm{~Hz}, 1 \mathrm{H}), 7.23(\mathrm{~d}, J=7.0 \mathrm{~Hz}, 1 \mathrm{H}), 7.13$ (t, $J=7.5 \mathrm{~Hz}, 1 \mathrm{H}), 6.85$ (s, 1H), $4.04(\mathrm{~d}, J=12.5 \mathrm{~Hz}, 1 \mathrm{H}), 3.85(\mathrm{~d}, J=12.5 \mathrm{~Hz}, 1 \mathrm{H}), 3.32(\mathrm{~d}, J=18.0 \mathrm{~Hz}, 1 \mathrm{H})$, $2.95(\mathrm{~d}, J=18.0 \mathrm{~Hz}, 1 \mathrm{H}), 1.26(\mathrm{~s}, 3 \mathrm{H}) ;{ }^{13} \mathbf{C ~ N M R}\left(125 \mathrm{MHz}, \mathrm{CDCl}_{3}\right) \delta=200.8$, $140.5,139.1,137.6,137.1,136.2,135.4,133.4,131.5,131.2,129.3,128.9,127.9$ (2C), 127.2, 124.3, 122.0, 120.8, 120.3, 109.0, 97.9, 56.6, 53.0, 36.2, 21.8. HRMS (ESI-TOF) m/z: $[\mathrm{M}+\mathrm{H}]^{+}$Calcd for $\mathrm{C}_{25} \mathrm{H}_{20} \mathrm{NO}^{+} 350.1539$; found 350.1541 . 


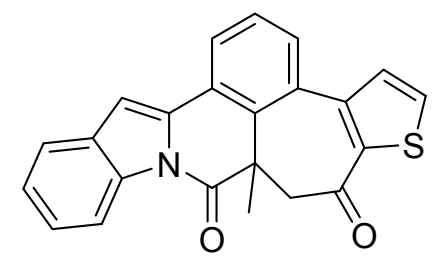

8a-methyl-8,8a-dihydroindolo $[2,1-a]$ thieno $\left[3^{\prime}, 2^{\prime}: 4,5\right]$ cyclohepta $[1,2,3-d e]$ isoquino line-7,9-dione (5al): yellow solid, isolated yield 51\% (37.6 mg) (eluent: petroleum ether/EtOAc = 10:1); mp: 224.6-225.3 ${ }^{\circ} \mathrm{C}$ (uncorrected); ${ }^{1} \mathbf{H}$ NMR (500 MHz, $\left.\mathrm{CDCl}_{3}\right)$ $\delta=8.57(\mathrm{~d}, J=8.0 \mathrm{~Hz}, 1 \mathrm{H}), 7.97-7.96(\mathrm{~m}, 1 \mathrm{H}), 7.74-7.71(\mathrm{~m}, 2 \mathrm{H}), 7.62(\mathrm{~d}, J=7.0 \mathrm{~Hz}$, 1H), 7.52 (t, $J=8.0 \mathrm{~Hz}, 1 \mathrm{H}), 7.43(\mathrm{~d}, J=5.0 \mathrm{~Hz}, 1 \mathrm{H}), 7.41-7.34(\mathrm{~m}, 2 \mathrm{H}), 7.10(\mathrm{~s}, 1 \mathrm{H})$, $3.99(\mathrm{~d}, J=17.5 \mathrm{~Hz}, 1 \mathrm{H}), 3.13(\mathrm{~d}, J=17.0 \mathrm{~Hz}, 1 \mathrm{H}), 1.63(\mathrm{~s}, 3 \mathrm{H}) ;{ }^{13} \mathrm{C}$ NMR $(125$ $\left.\mathrm{MHz}, \mathrm{CDCl}_{3}\right) \delta=190.4,171.9,143.5,139.5,136.0,135.2,134.6,133.4,133.2,131.6$, $130.7,130.7,128.3,125.5,125.0,124.8,123.9,120.6,116.9,104.0,50.9,44.6,22.5$. HRMS (ESI-TOF) m/z: [M + H] $]^{+}$Calcd for $\mathrm{C}_{23} \mathrm{H}_{16} \mathrm{NO}_{2} \mathrm{~S}^{+}$370.0896; found 370.0899. 


\section{References}

1. Kong, W.; Casimiro, M.; Fuentes, N.; Merino, E.; Nevado, C. Angew. Chem. Int. $E d, \mathbf{2 0 1 3}, 52,13086$.

2. Sharma, U. K.; Sharma, N.; Kumar, Y.; Singh, B. K.; Van der Eycken, E. V. Chem. Eur. J. 2016, 22, 481.

3. Whyte, A.; Olson, M. E.; Lautens, M. Org. Lett. 2018, 20, 345.

4. Pérez-Gómez, M.; García-López, J.-A. Angew. Chem. Int. Ed. 2016, 55, 14389.

5. Takeda, Y.; Okumura, S.; Tone, S.; Sasaki, I.; Minakata, S. Org. Lett. 2012, 14, 4874 .

6. Yang, X.; Lu, H.; Zhu, X.; Zhou, L.; Deng, G.; Yang, Y.; Liang, Y. Org. Lett. 2019, 21, 7284 .

7. Wu, L.; Deng, G.; Liang, Y. Org. Biomol. Chem. 2017, 15, 6808.

8. Wei, Y.-L.; Chen, J.-Q.; Sun, B.; Xu, P.-F. Chem. Commun. 2019, 55, 5922.

9. Wipf, P.; Maciejewski, J. Org. Lett. 2008, 10, 4383.

10. Mahendar, 1.; Krishna, J.; Alavala, GKR.; Venkat Ramulu, B.; Satyanarayana, G. Org. Lett. 2012, 14, 628.

11. Fu, J.; Han, J.; Meng, T.; Hu, J.; Yin, J. Chem. Commun. 2019, 55, 12904. 


\section{Scanned ${ }^{1} \mathrm{H}$ NMR and ${ }^{13} \mathrm{C}$ NMR Spectra of All Compounds}

${ }^{1} \mathrm{H}$ NMR of $3 \mathbf{a a}\left(500 \mathrm{MHz}, \mathrm{CDCl}_{3}\right)$ and ${ }^{13} \mathrm{C} \mathrm{NMR} \mathrm{of} \mathbf{3 a a}\left(125 \mathrm{MHz}, \mathrm{CDCl}_{3}\right)$

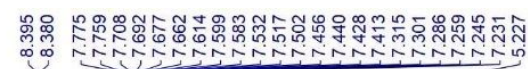



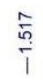
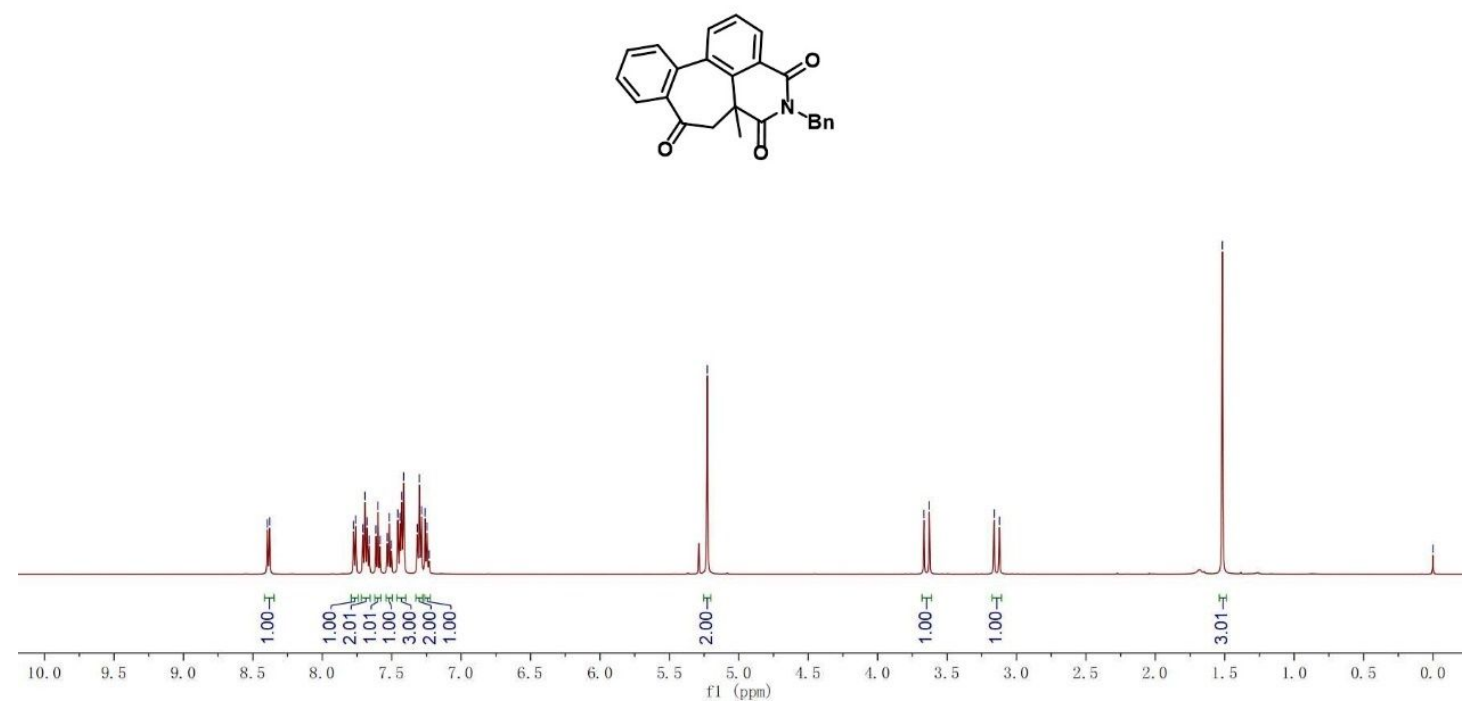

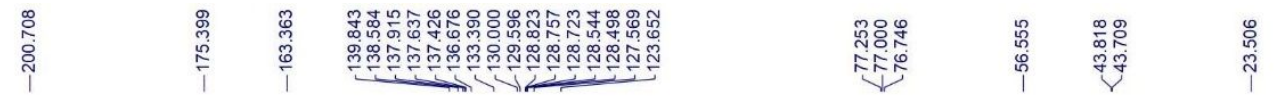
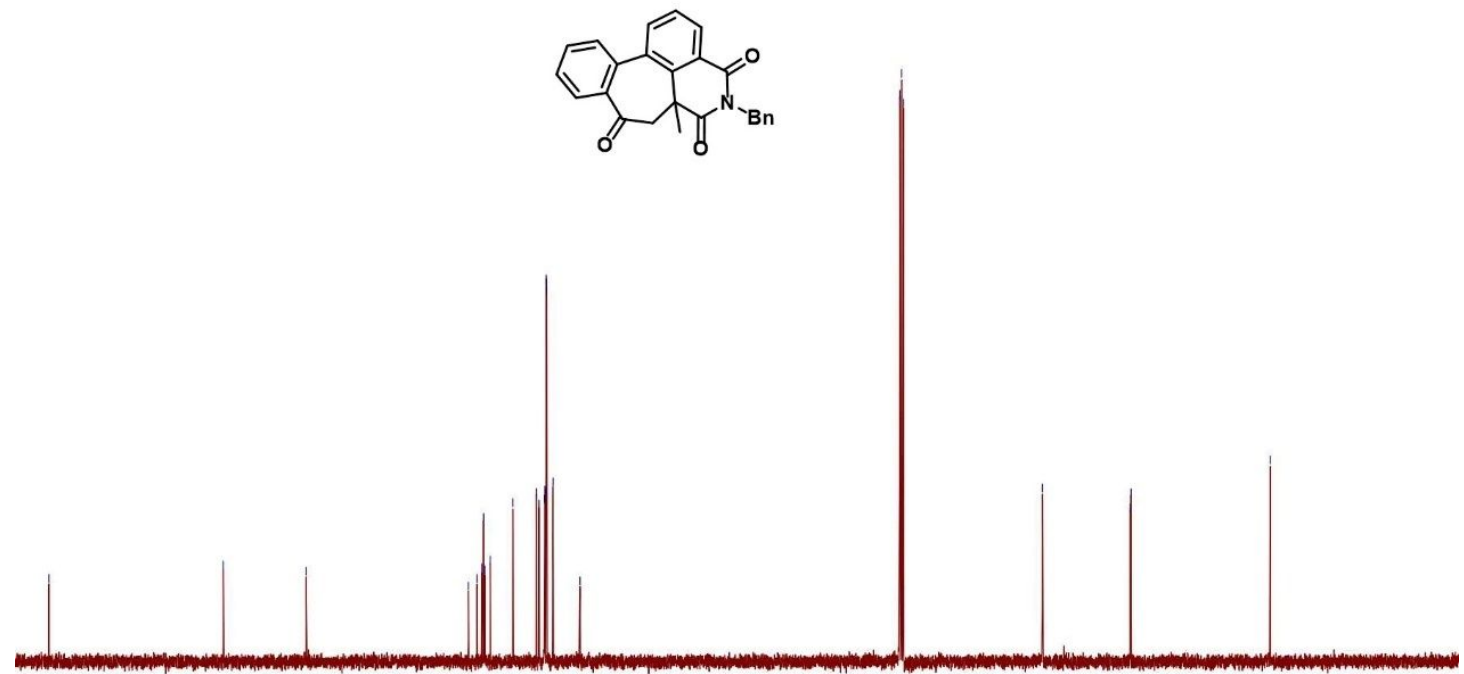

$\begin{array}{lllllllllll}200 & 190 & 180 & 170 & 160 & 150 & 140 & 130 & 120 & 110 & 100 \\ \mathrm{fl} & (\mathrm{pmm})\end{array}$ 
${ }^{1} \mathrm{H} \mathrm{NMR}$ of $3 \mathbf{b a}\left(500 \mathrm{MHz}, \mathrm{CDCl}_{3}\right)$ and ${ }^{13} \mathrm{C} \mathrm{NMR}$ of $3 \mathbf{b a}\left(125 \mathrm{MHz}, \mathrm{CDCl}_{3}\right)$

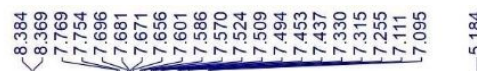

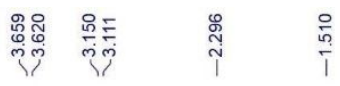
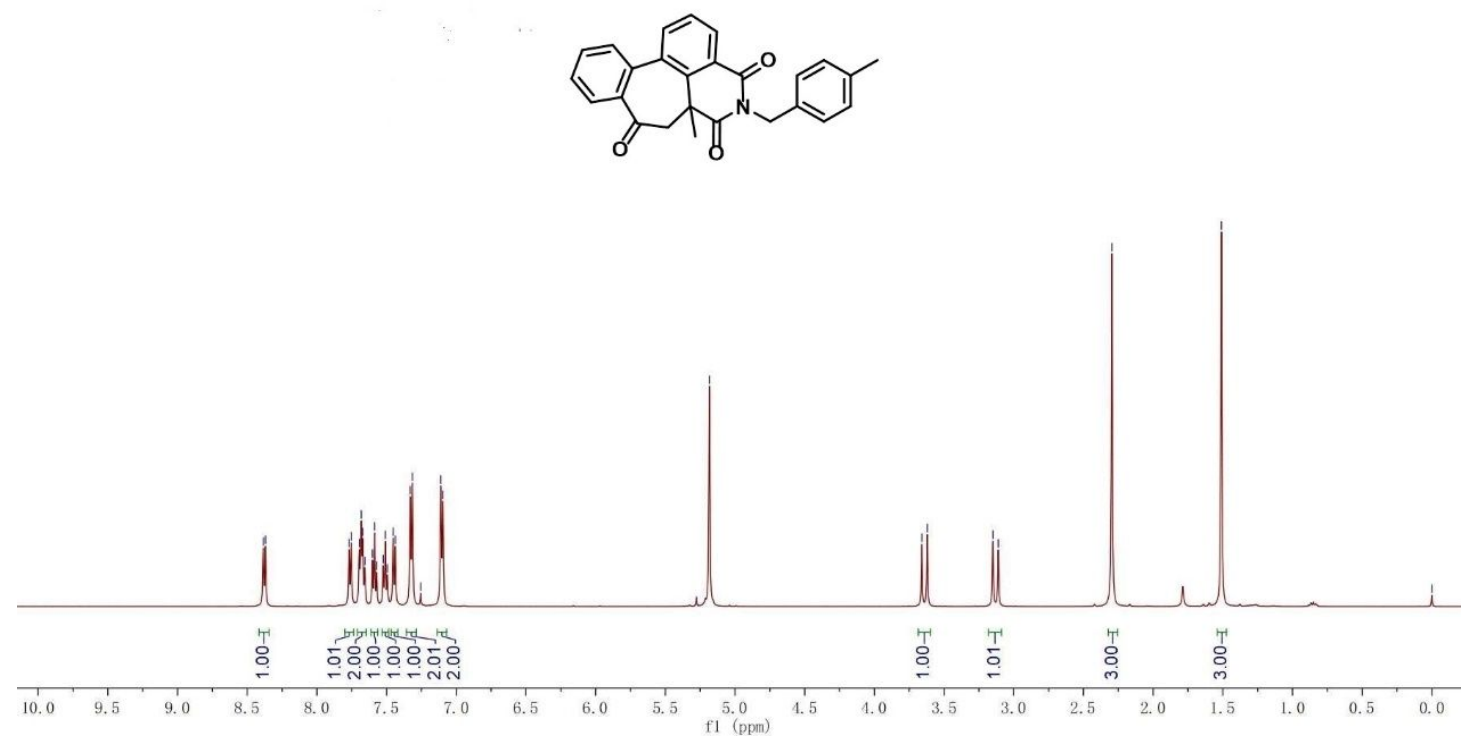

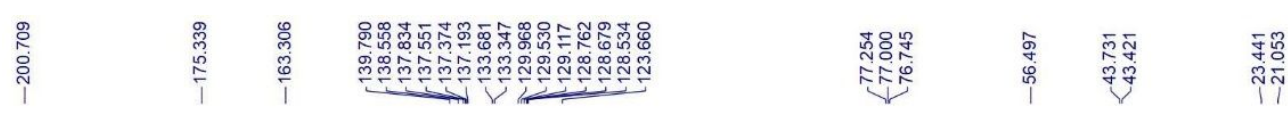
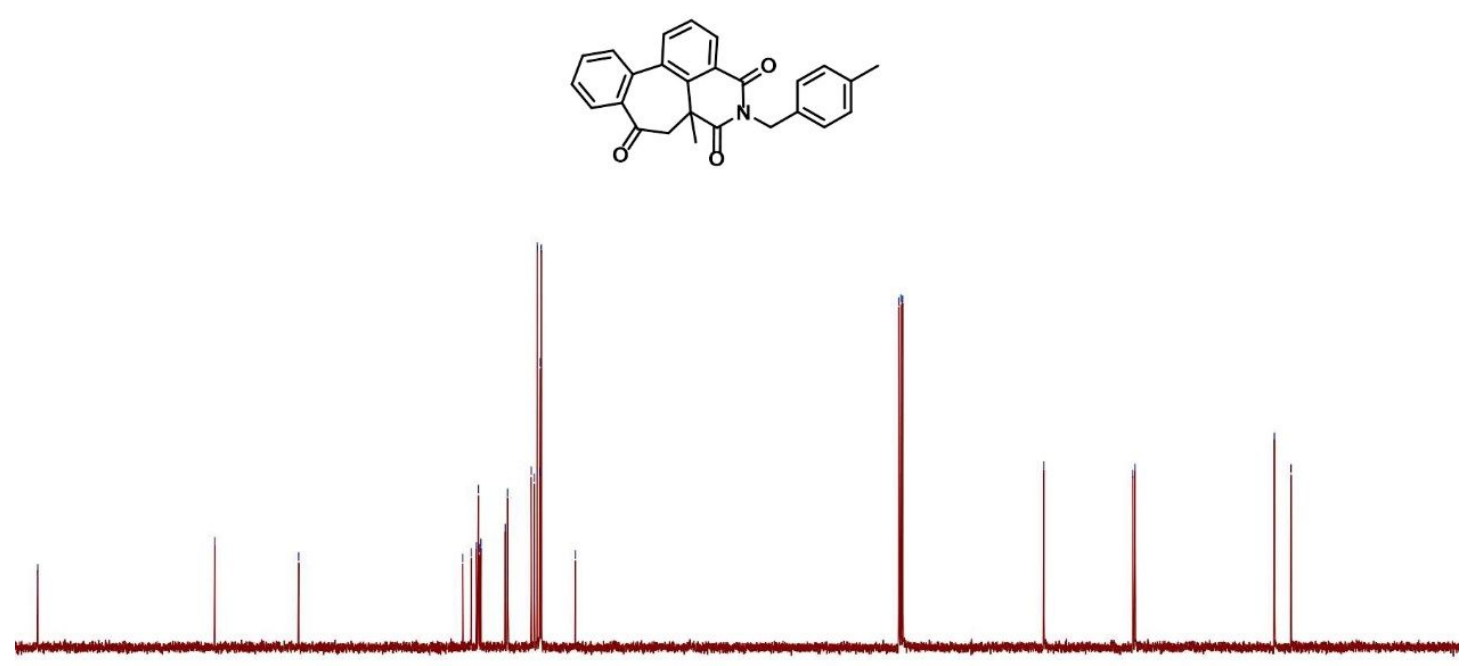

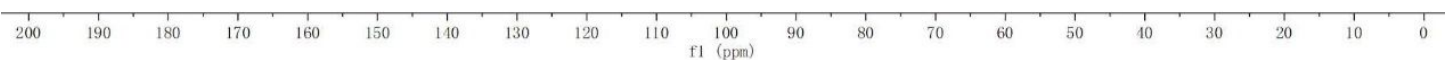


${ }^{1} \mathrm{H}$ NMR of 3ca $\left(500 \mathrm{MHz}, \mathrm{CDCl}_{3}\right)$ and ${ }^{13} \mathrm{C}$ NMR of $3 \mathbf{c a}\left(125 \mathrm{MHz}, \mathrm{CDCl}_{3}\right)$

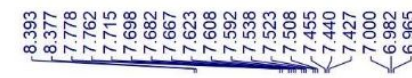<smiles>CC12CC(=O)c3ccccc3-c3cccc(c31)C(=O)N(Cc1ccc(F)cc1)C2=O</smiles>

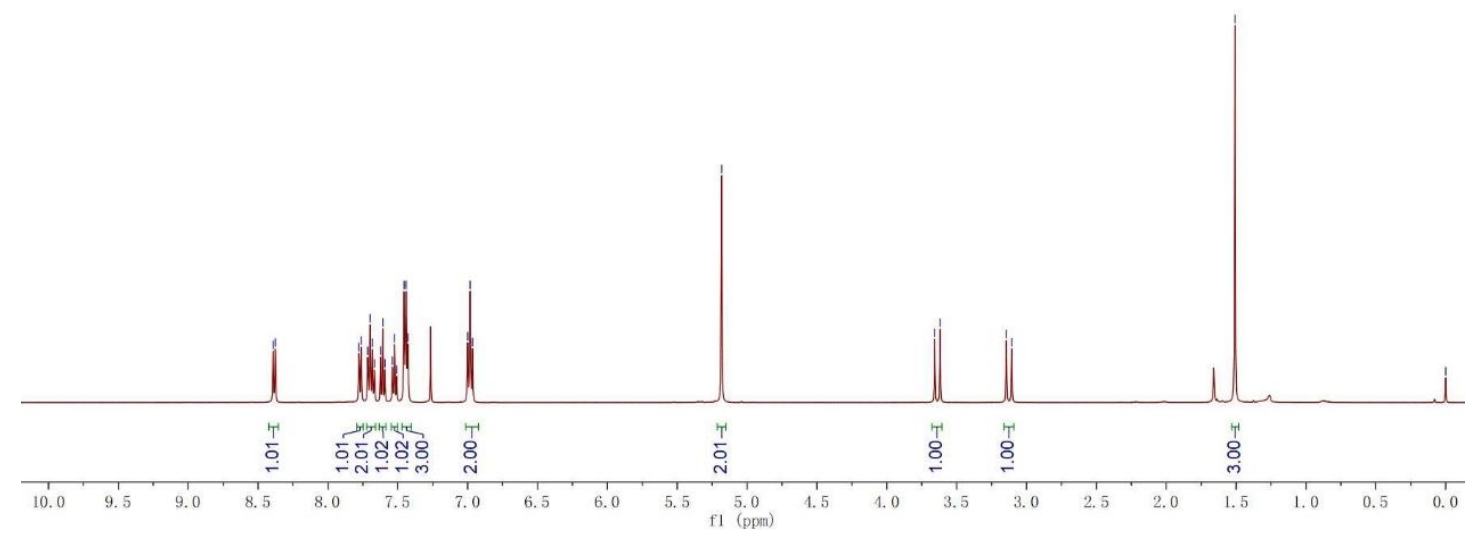

亯<smiles>CC12CC(=O)c3ccccc3-c3cccc(c31)C(=O)N(Cc1ccc(F)cc1)C2=O</smiles>

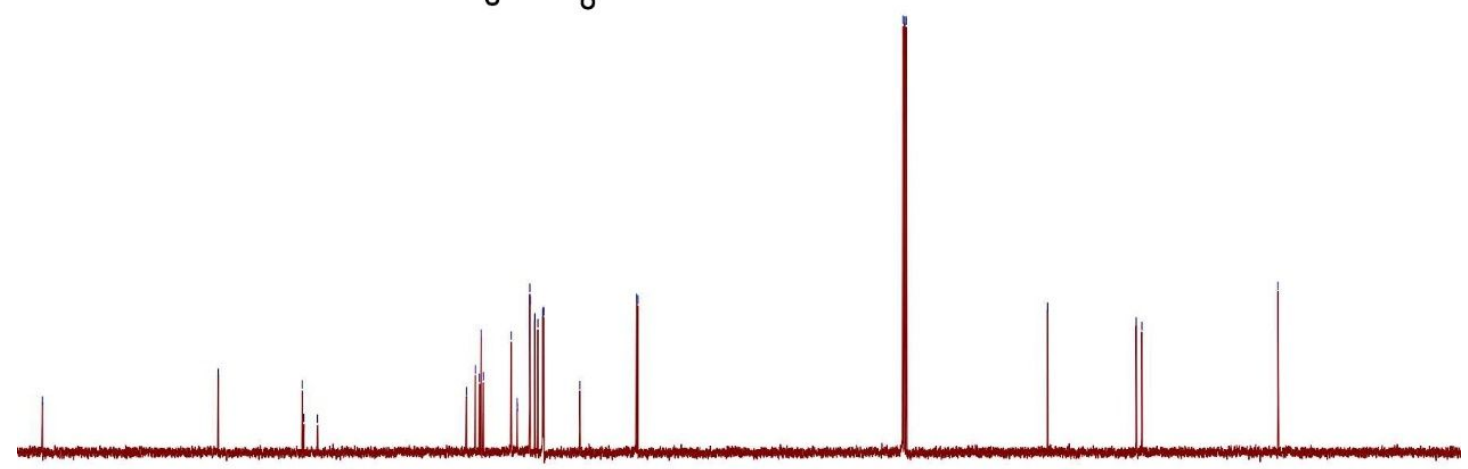

$200 \quad 190 \quad 18$ $170 \quad 160$ $150 \quad 140$ 
${ }^{1} \mathrm{H} \mathrm{NMR}$ of 3da $\left(500 \mathrm{MHz}, \mathrm{CDCl}_{3}\right)$ and ${ }^{13} \mathrm{C} \mathrm{NMR}$ of $3 \mathbf{d a}\left(125 \mathrm{MHz}, \mathrm{CDCl}_{3}\right)$

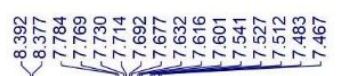

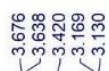

$\stackrel{\text { ing }}{\text { i }}$

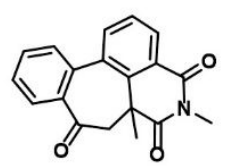

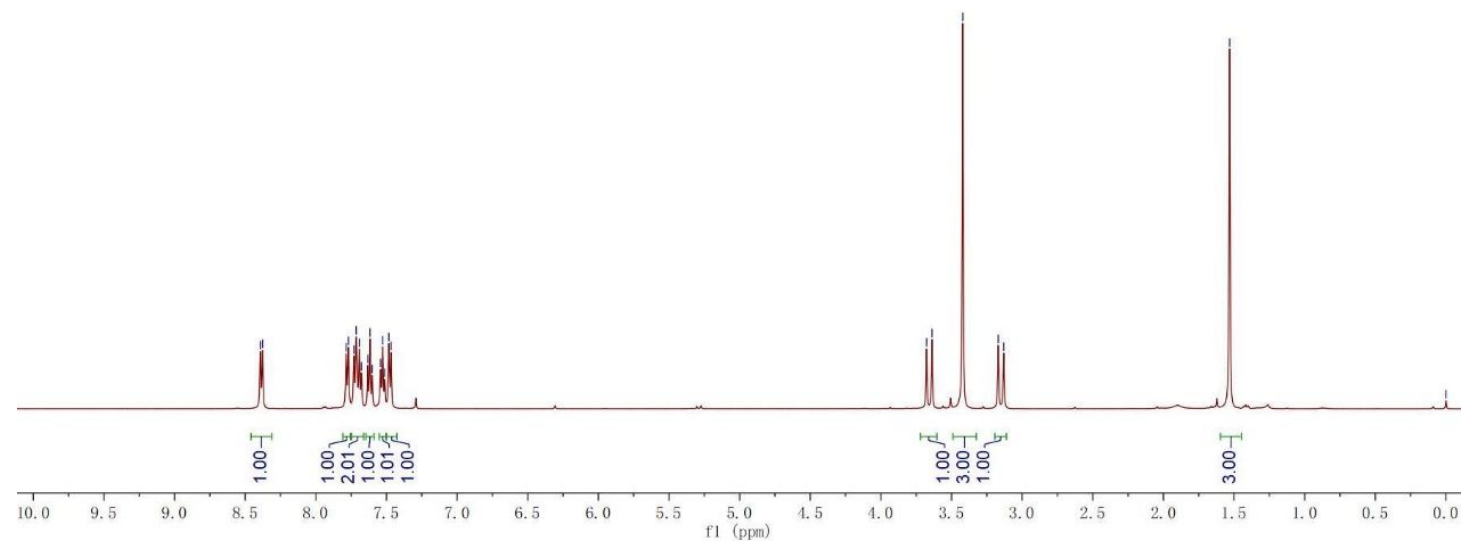

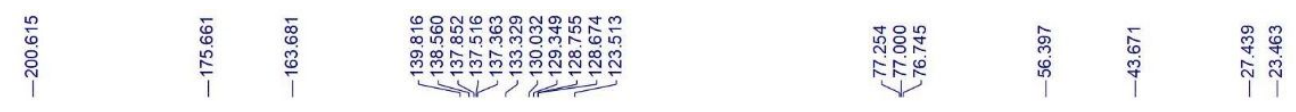<smiles>CN1C(=O)C23C=CC=C2C2(C=CC=C2)C(=O)C(=O)N1C3=O</smiles>
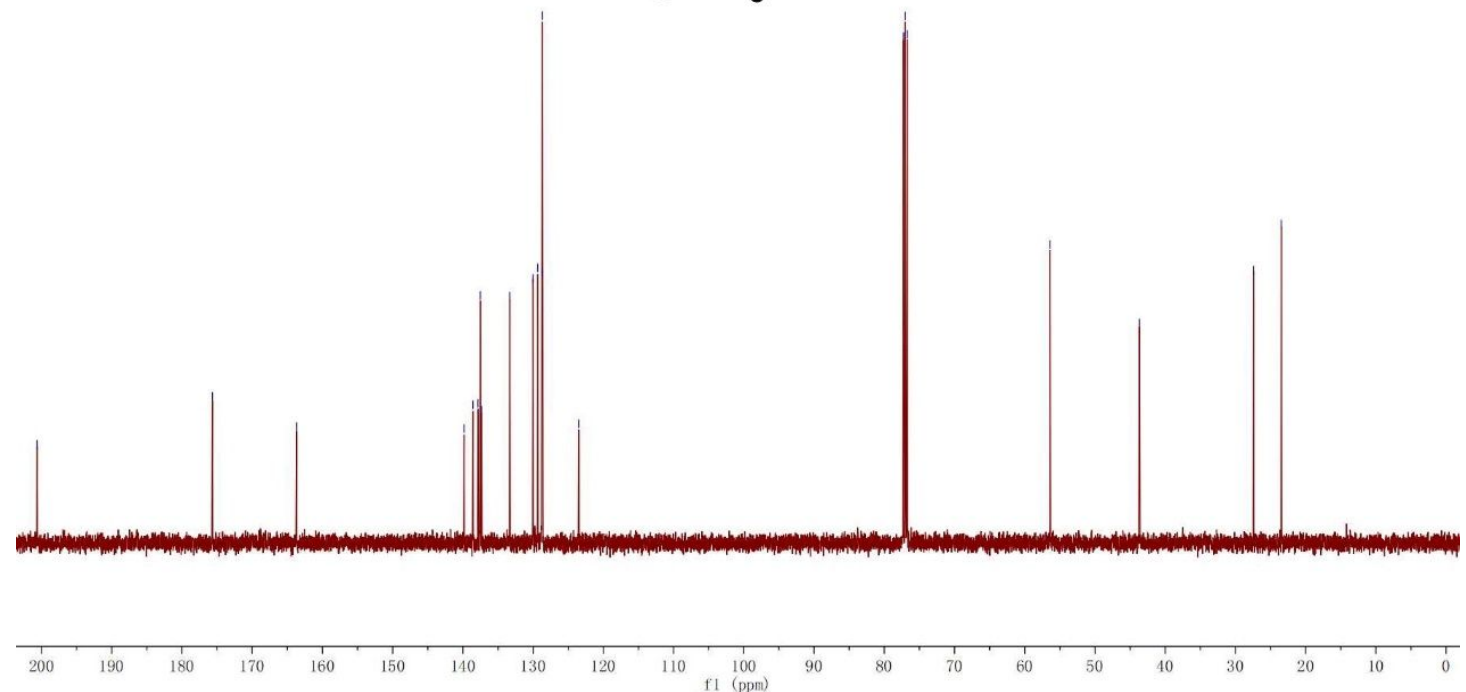
${ }^{1} \mathrm{H}$ NMR of 3ea $\left(500 \mathrm{MHz}, \mathrm{CDCl}_{3}\right)$ and ${ }^{13} \mathrm{C} \mathrm{NMR} \mathrm{of} \mathbf{3 e a}\left(125 \mathrm{MHz}, \mathrm{CDCl}_{3}\right)$

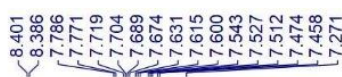

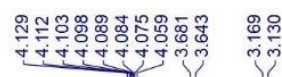
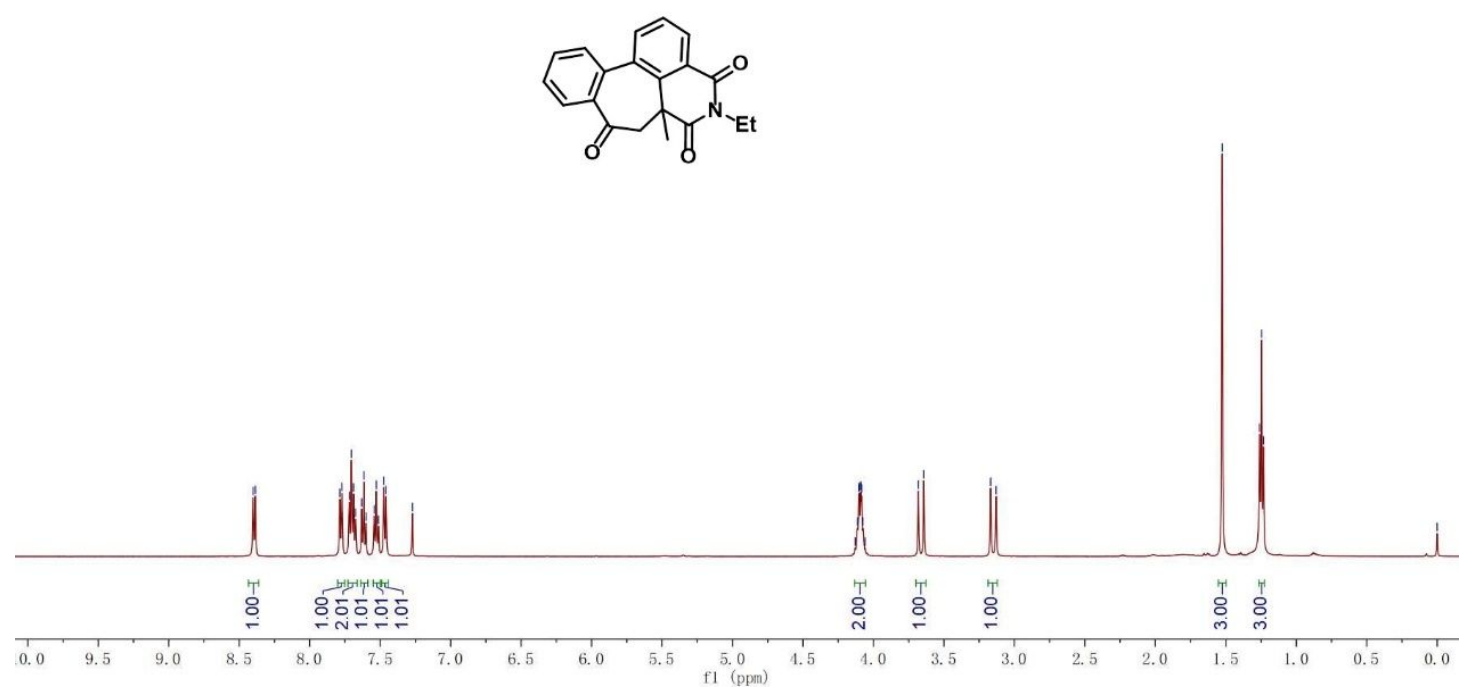

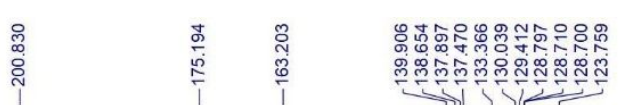

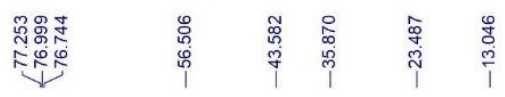
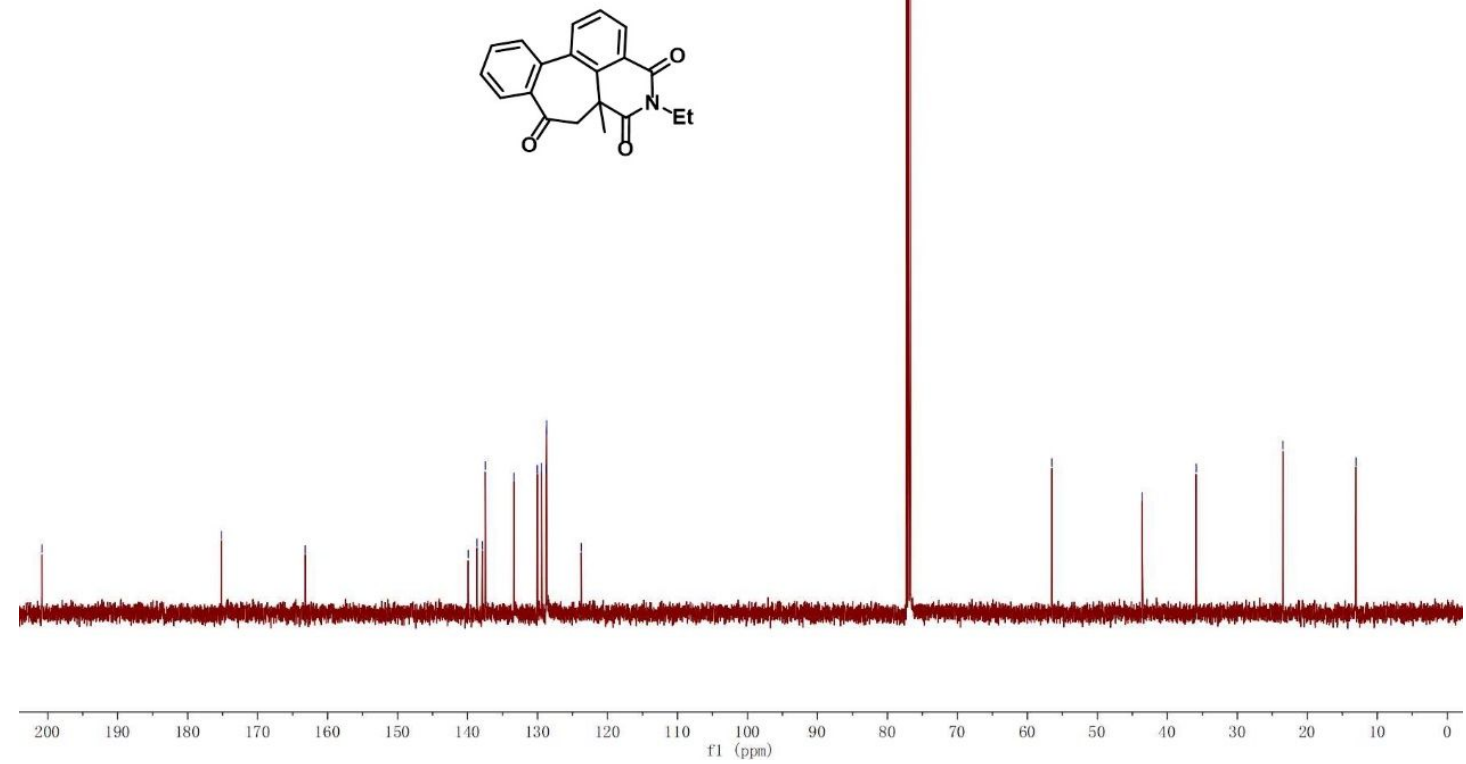
${ }^{1} \mathrm{H}$ NMR of $\mathbf{3 f a}\left(500 \mathrm{MHz}, \mathrm{CDCl}_{3}\right)$ and ${ }^{13} \mathrm{C} \mathrm{NMR}$ of $\mathbf{3 f a}\left(125 \mathrm{MHz}, \mathrm{CDCl}_{3}\right)$





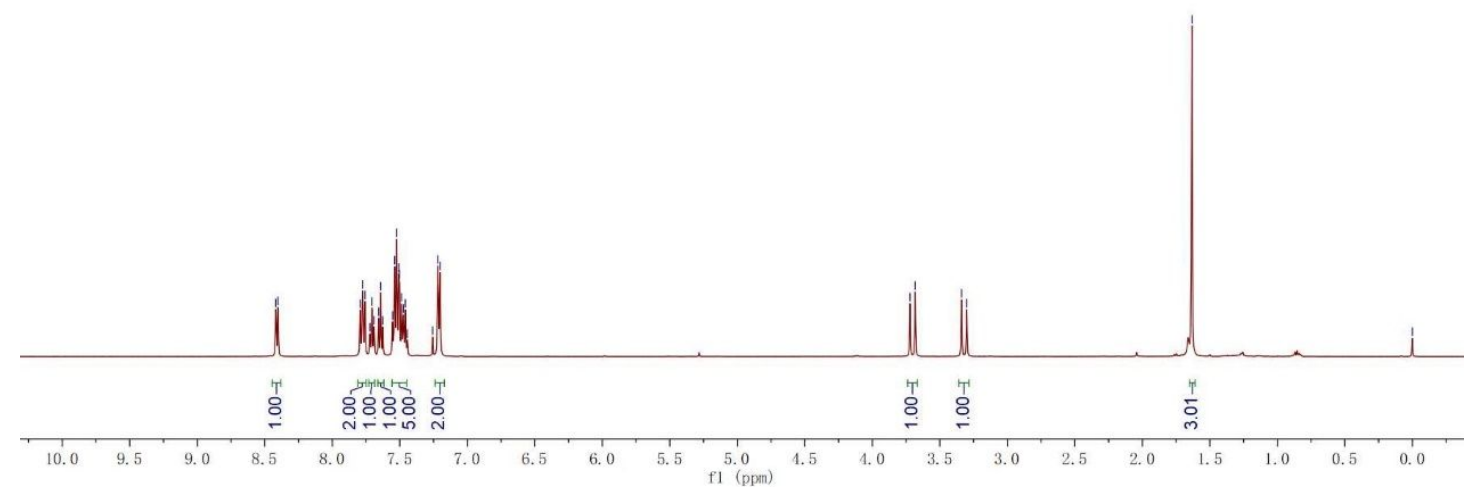

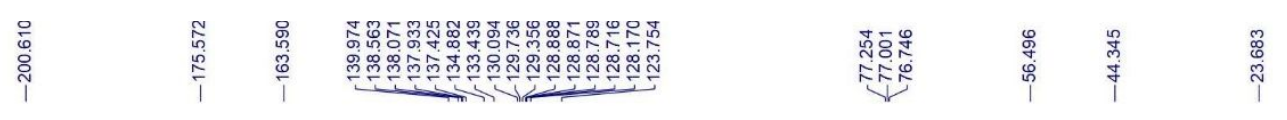

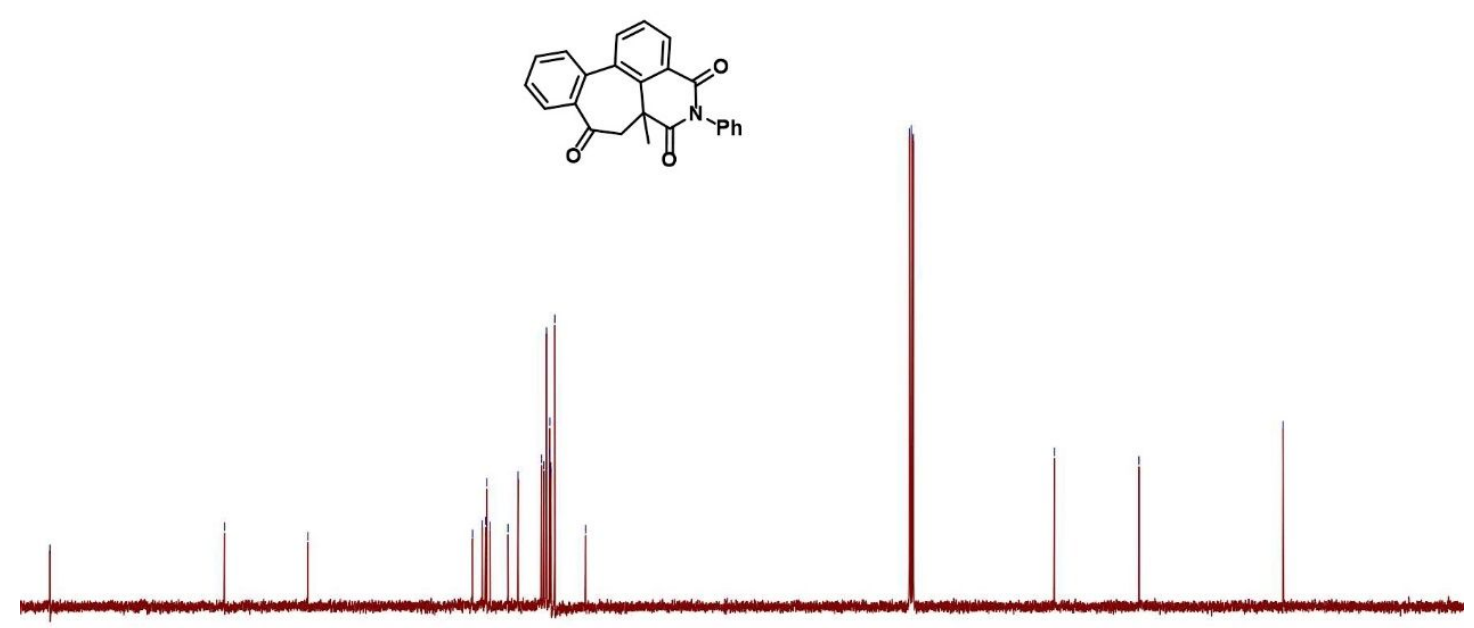

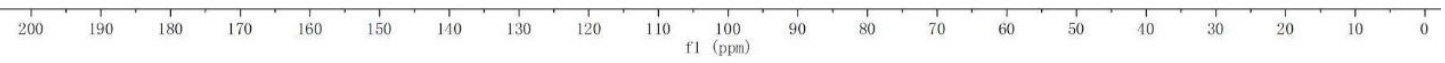


${ }^{1} \mathrm{H} \mathrm{NMR}$ of 3 ga $\left(500 \mathrm{MHz}, \mathrm{CDCl}_{3}\right)$ and ${ }^{13} \mathrm{C} \mathrm{NMR}$ of 3 ga $\left(125 \mathrm{MHz}, \mathrm{CDCl}_{3}\right)$

<smiles>C=CCN1C(=O)c2cccc3c2C(CC(=O)C2C=CC=C32)C1=O</smiles>

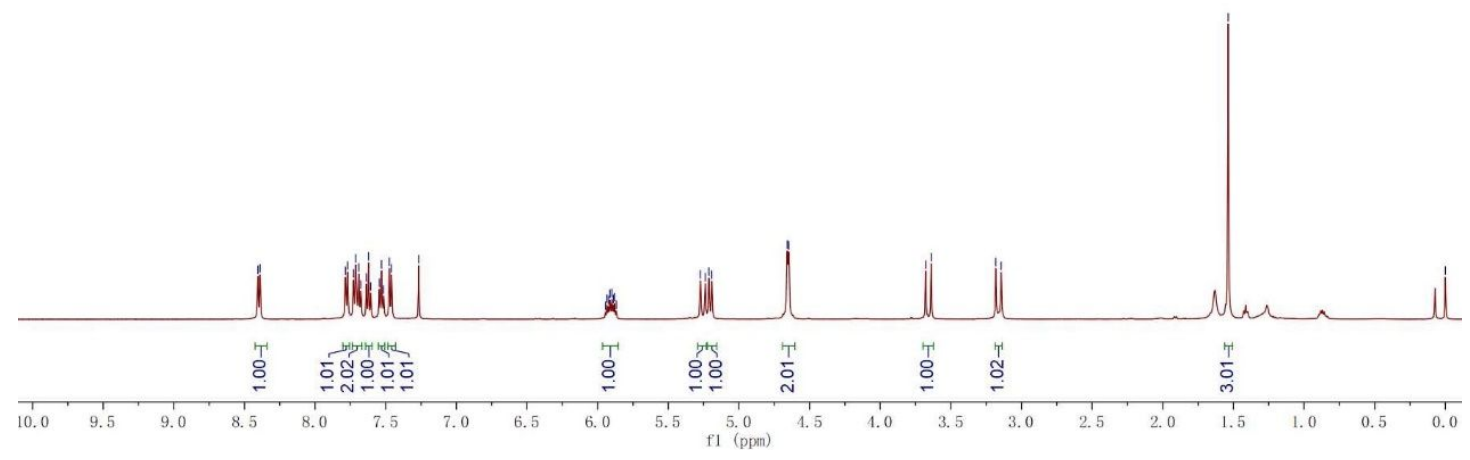

<smiles>C=CCN1C(=O)c2cccc3c2C(CC(=O)C2C=CC=C32)C1=O</smiles>

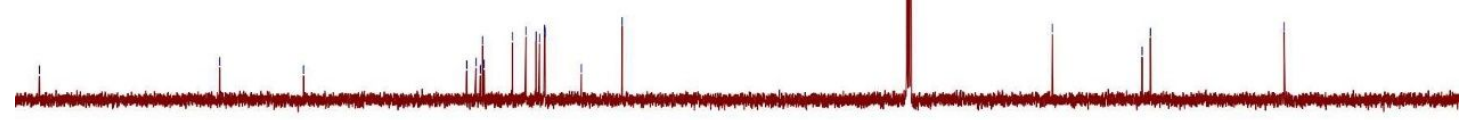

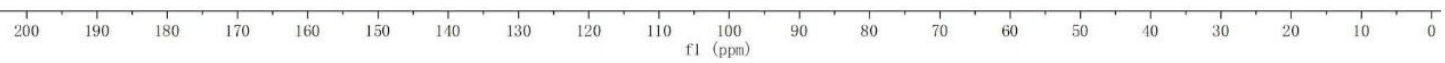


${ }^{1} \mathrm{H}$ NMR of 3 ha $\left(500 \mathrm{MHz}, \mathrm{CDCl}_{3}\right)$ and ${ }^{13} \mathrm{C} \mathrm{NMR}$ of $\mathbf{3 h a}\left(125 \mathrm{MHz}, \mathrm{CDCl}_{3}\right)$

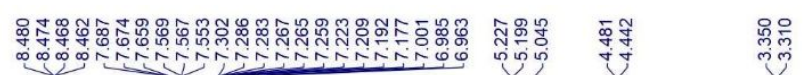

${ }^{1} \mathrm{H}$ NMR of 3ia $\left(500 \mathrm{MHz}, \mathrm{CDCl}_{3}\right)$ and ${ }^{13} \mathrm{C}$ NMR of $3 \mathbf{i a}\left(125 \mathrm{MHz}, \mathrm{CDCl}_{3}\right)$

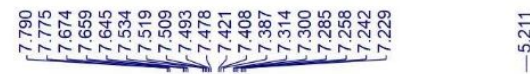

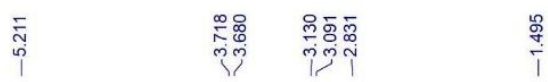
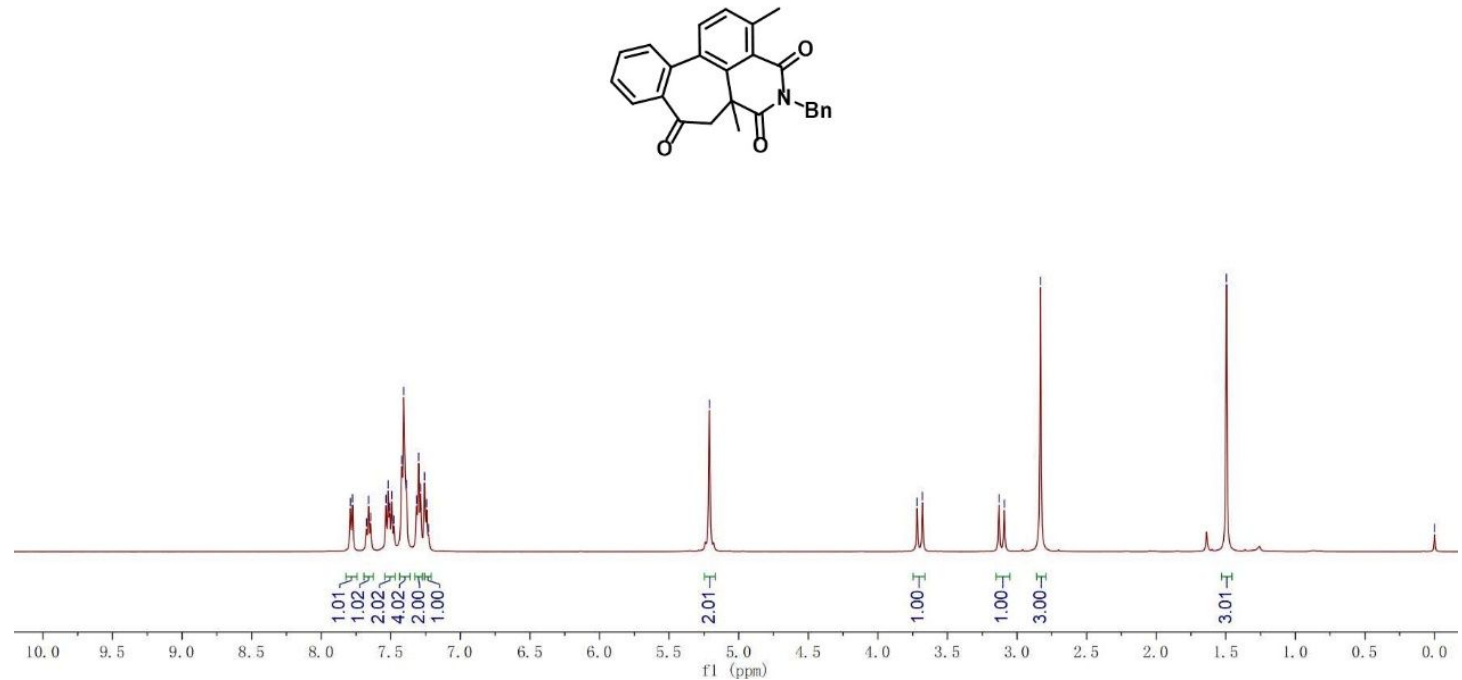



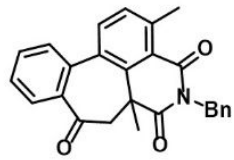

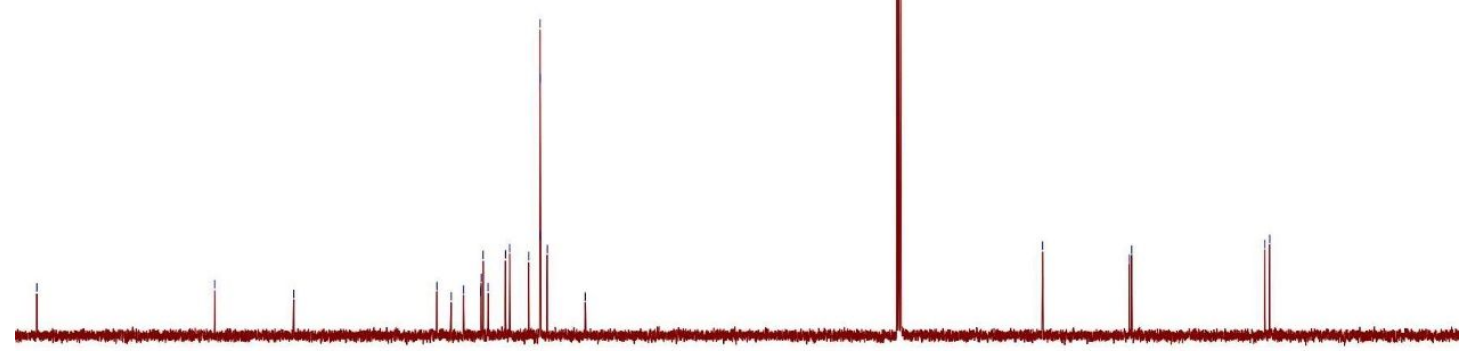

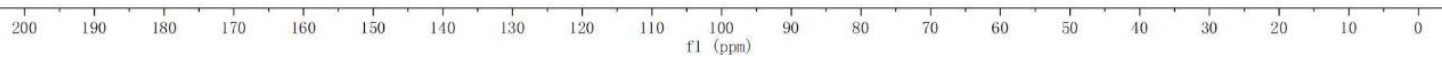


${ }^{1} \mathrm{H}$ NMR of $\mathbf{3 j a}\left(500 \mathrm{MHz}, \mathrm{CDCl}_{3}\right)$ and ${ }^{13} \mathrm{C}$ NMR of $\mathbf{3 j a}\left(125 \mathrm{MHz}, \mathrm{CDCl}_{3}\right)$

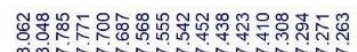
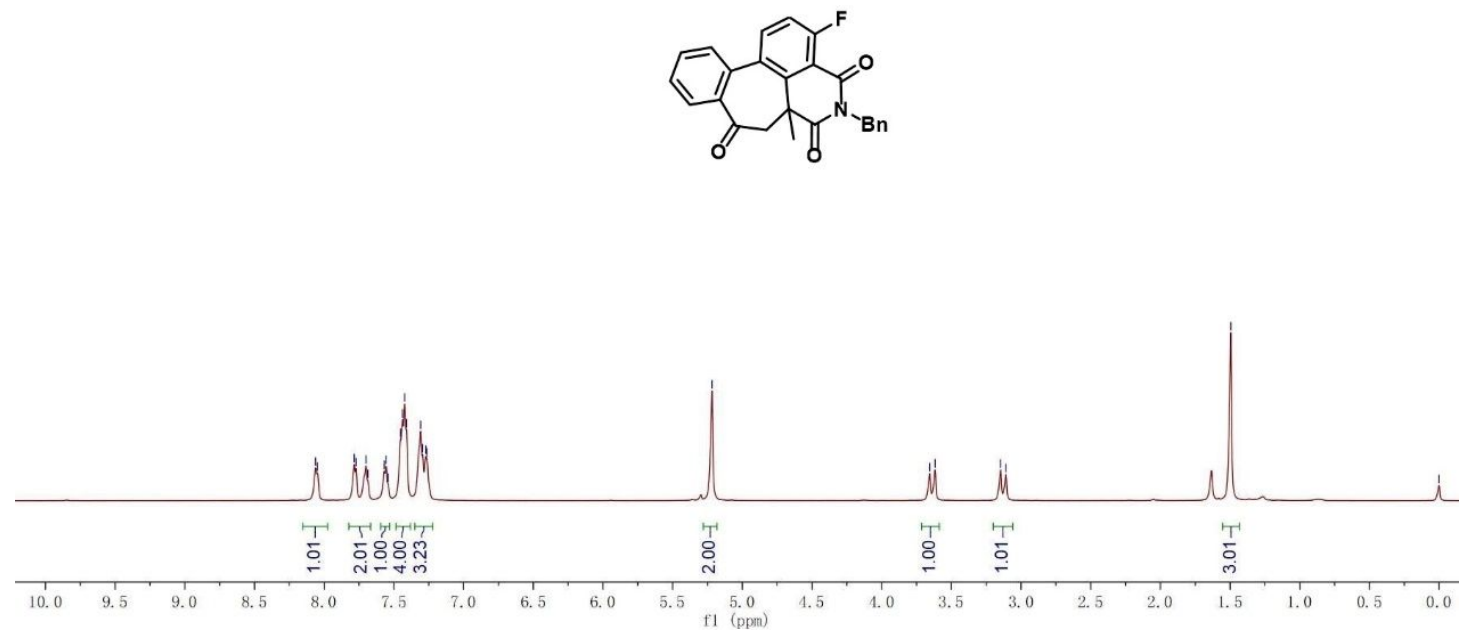

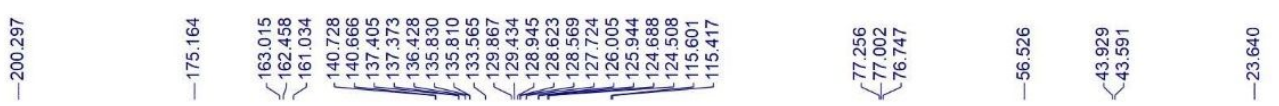
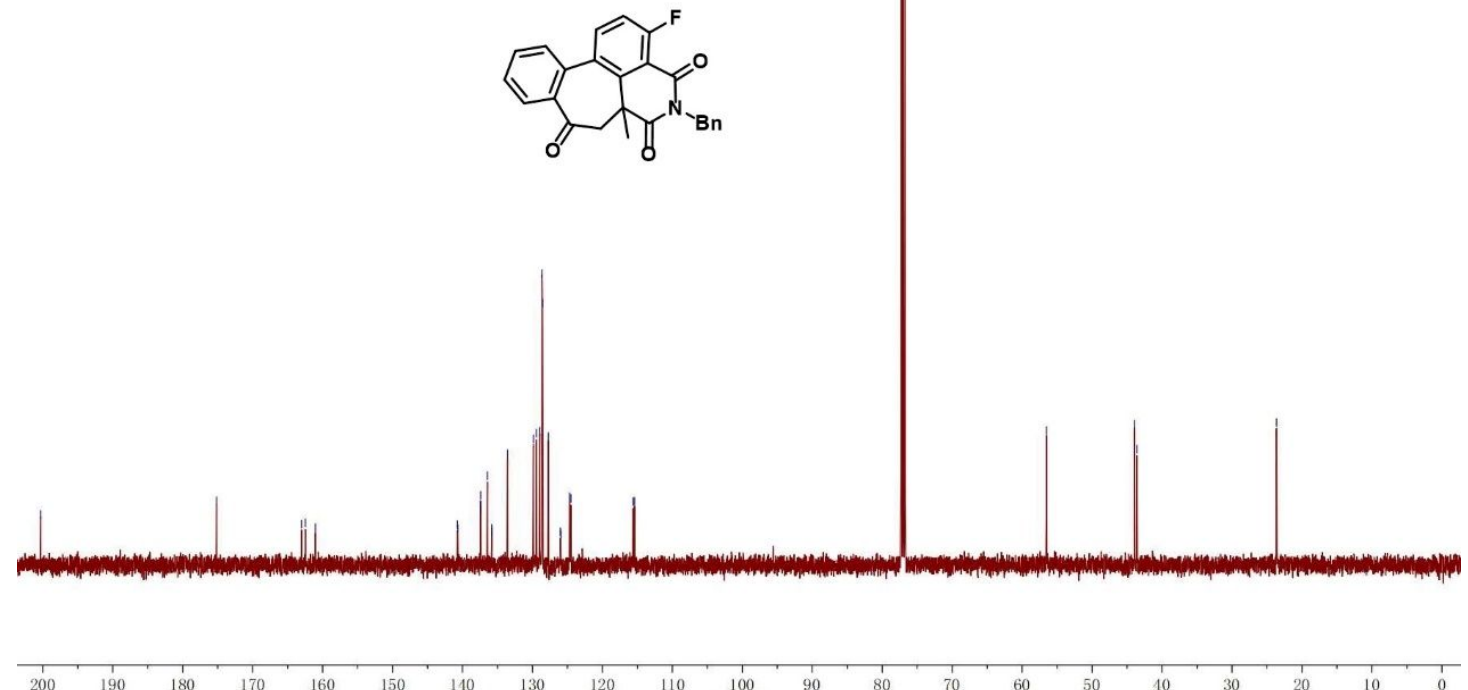
$10 \quad \begin{aligned} & 100 \\ & \text { fl }(\mathrm{ppm})\end{aligned}$ 
${ }^{1} \mathrm{H}$ NMR of $3 \mathbf{k a}\left(500 \mathrm{MHz}, \mathrm{CDCl}_{3}\right)$ and ${ }^{13} \mathrm{C}$ NMR of $\mathbf{3 k a}\left(125 \mathrm{MHz}, \mathrm{CDCl}_{3}\right)$

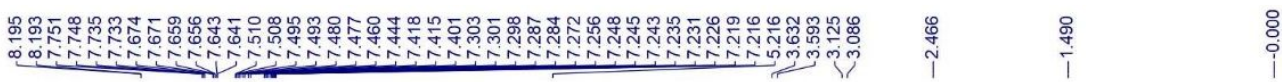

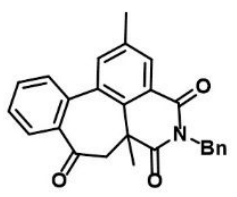





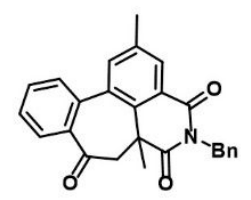
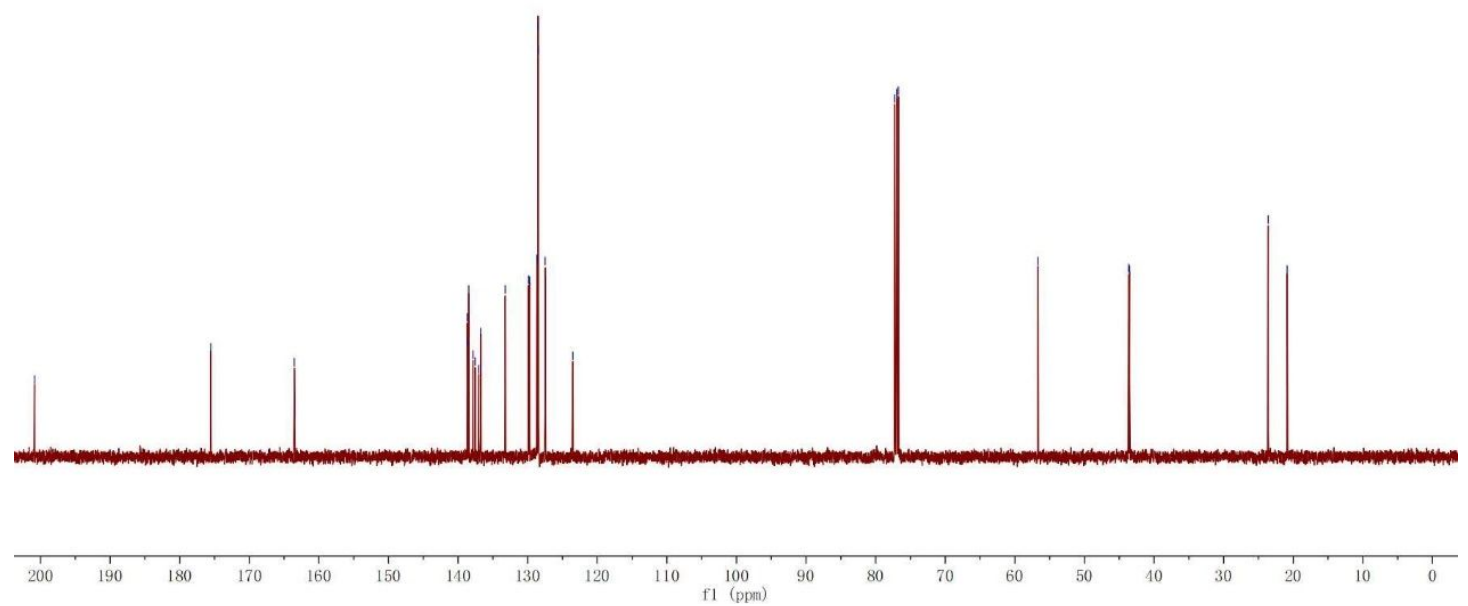
${ }^{1} \mathrm{H}$ NMR of 3la $\left(500 \mathrm{MHz}, \mathrm{CDCl}_{3}\right)$ and ${ }^{13} \mathrm{C} \mathrm{NMR} \mathrm{of} 31 \mathbf{a}\left(125 \mathrm{MHz}, \mathrm{CDCl}_{3}\right)$

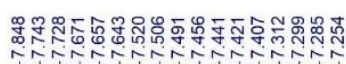



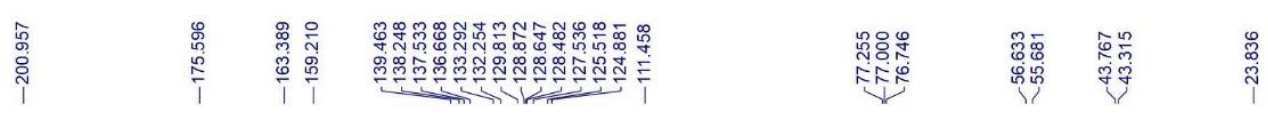
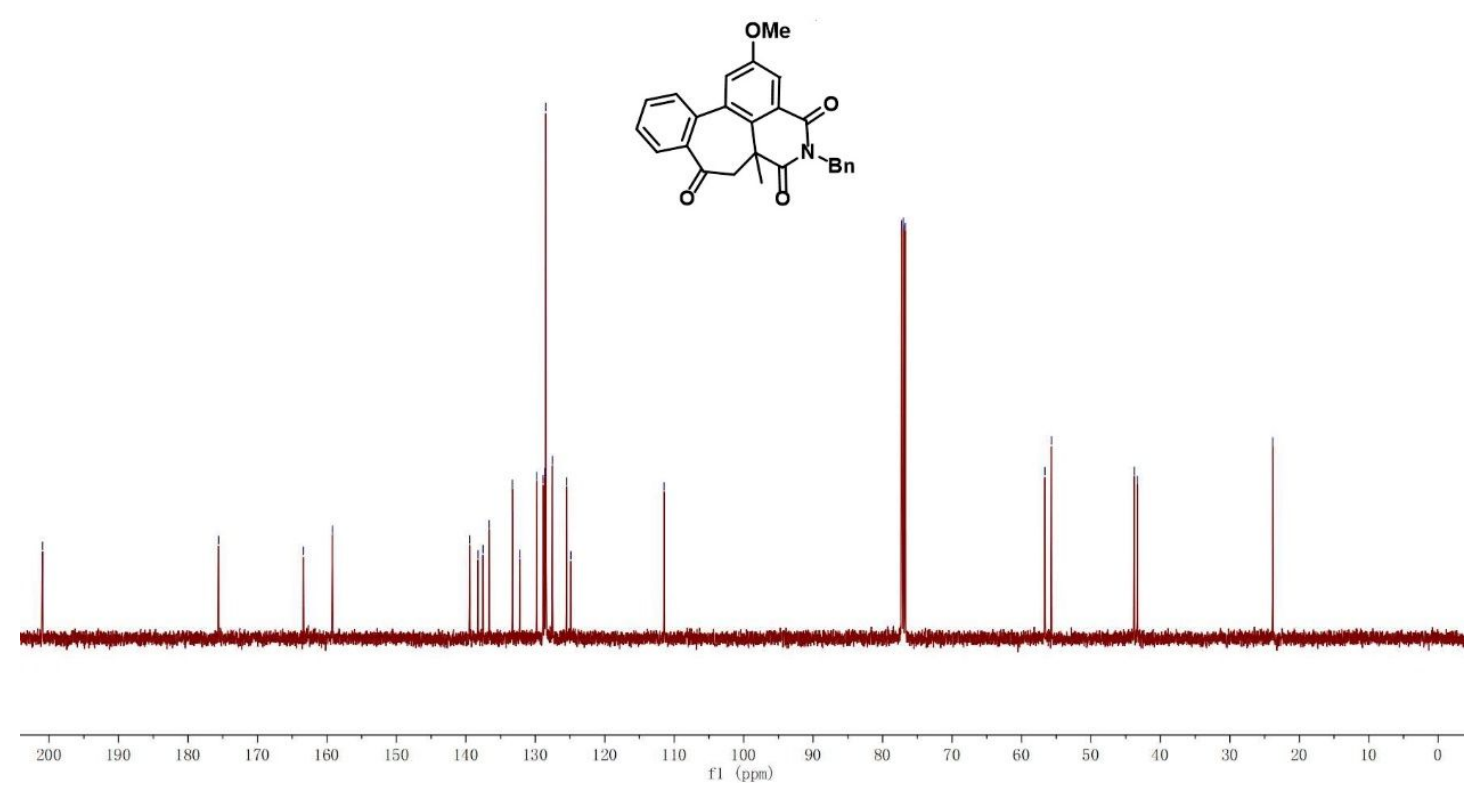
${ }^{1} \mathrm{H}$ NMR of 3 ma $\left(500 \mathrm{MHz}, \mathrm{CDCl}_{3}\right)$ and ${ }^{13} \mathrm{C} \mathrm{NMR}$ of 3 ma $\left(125 \mathrm{MHz}, \mathrm{CDCl}_{3}\right)$

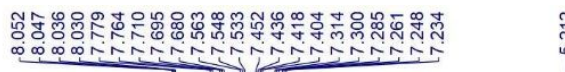

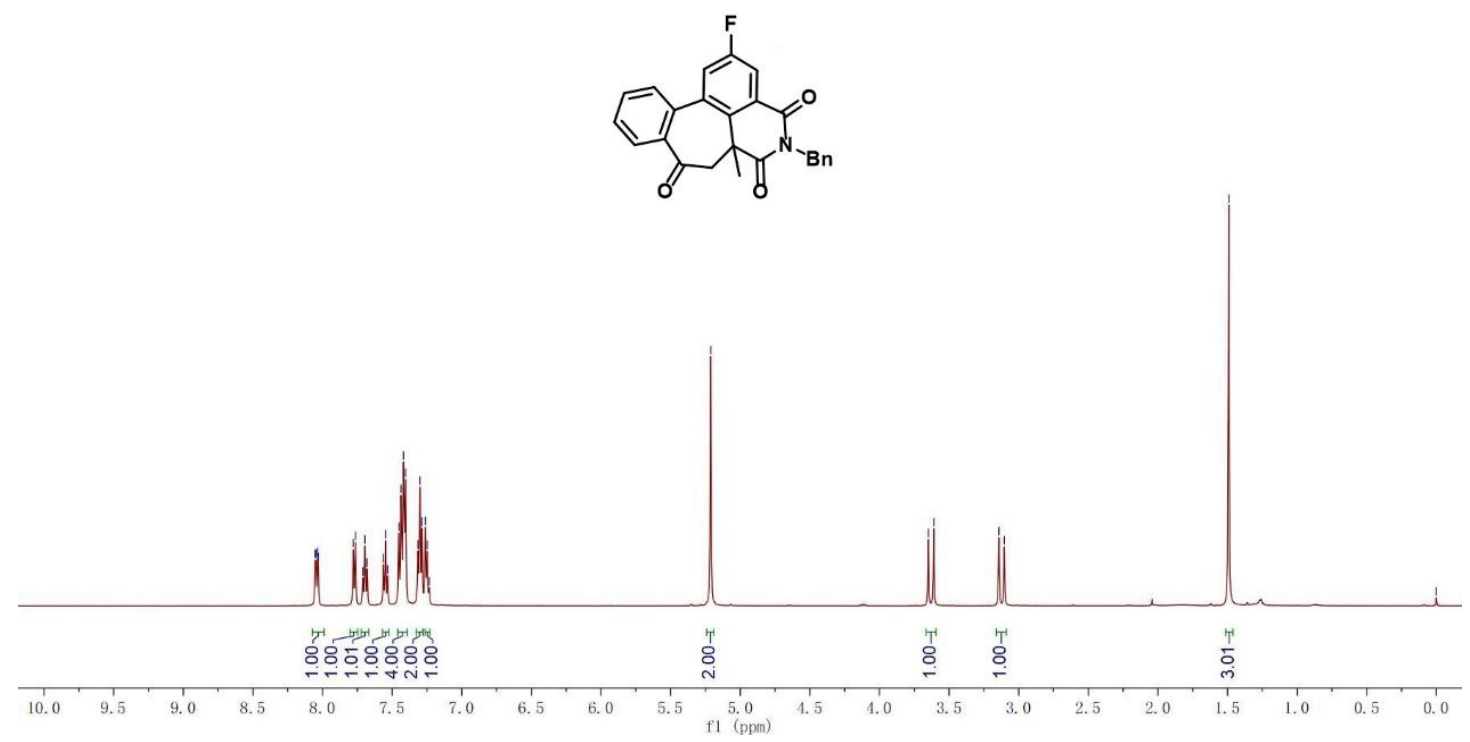

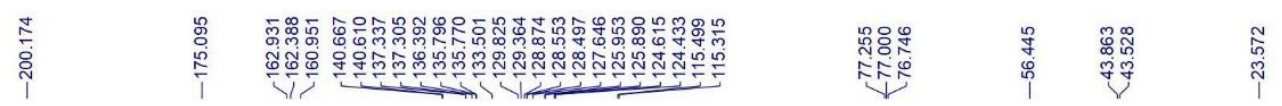

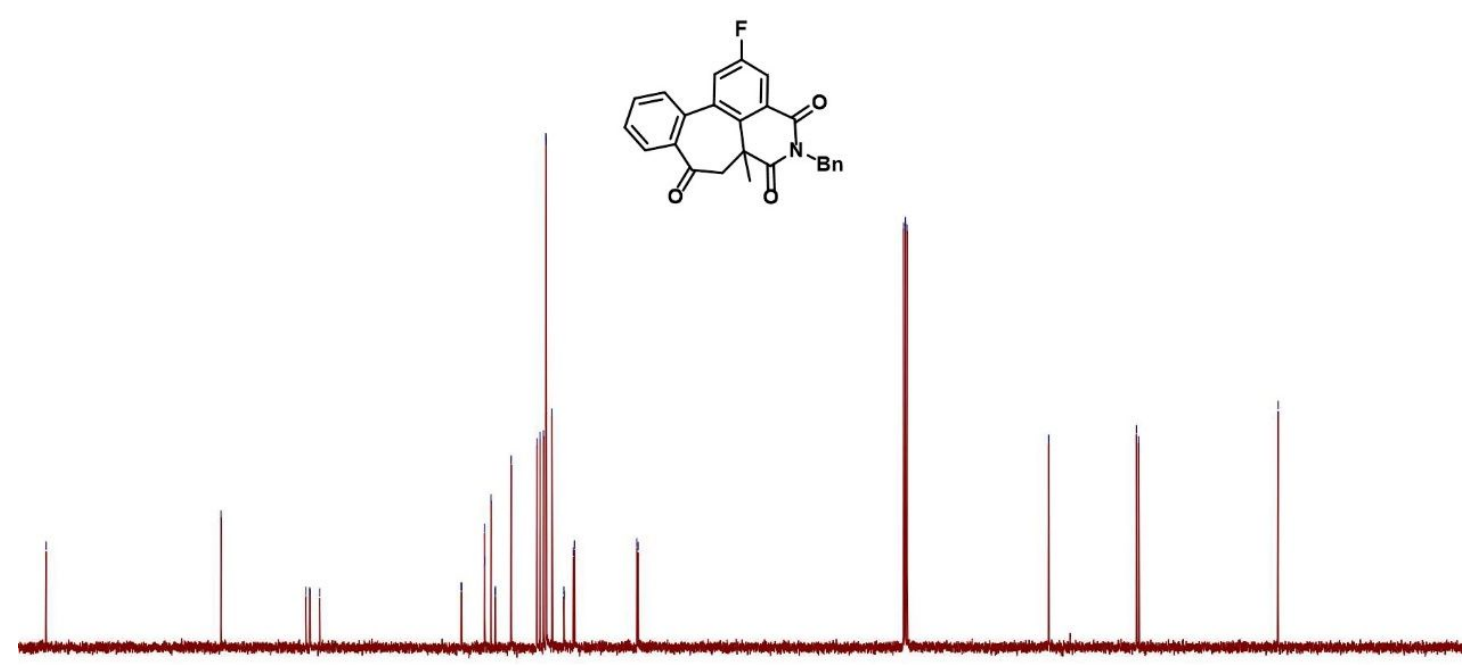

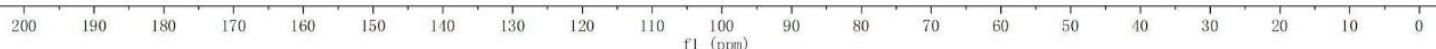


${ }^{1} \mathrm{H}$ NMR of 3na (500 MHz, $\left.\mathrm{CDCl}_{3}\right)$ and ${ }^{13} \mathrm{C}$ NMR of 3na (125 MHz, $\left.\mathrm{CDCl}_{3}\right)$

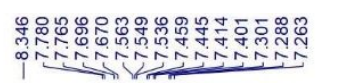

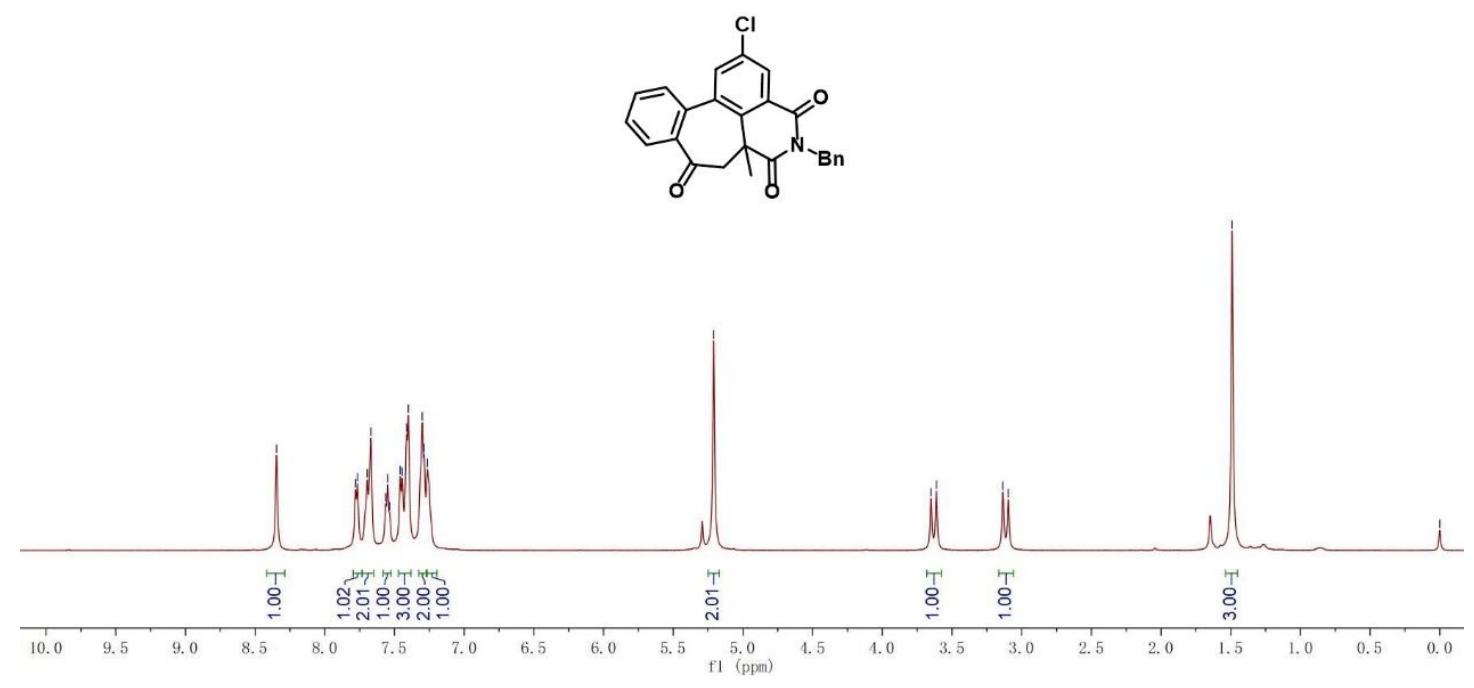

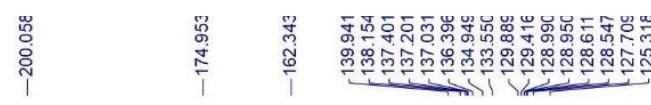

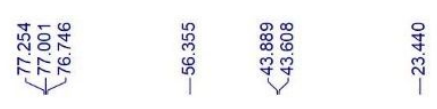<smiles>CC12CC(=O)c3ccccc3-c3cc(Cl)cc(c31)C(=O)N(Cc1ccccc1)C2=O</smiles>

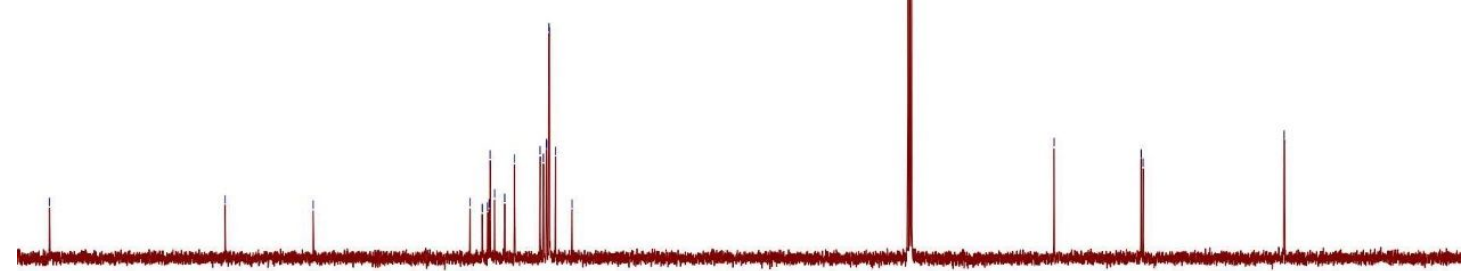


${ }^{1} \mathrm{H} \mathrm{NMR}$ of 3 pa $\left(500 \mathrm{MHz}, \mathrm{CDCl}_{3}\right)$ and ${ }^{13} \mathrm{C} \mathrm{NMR}$ of 3pa $\left(125 \mathrm{MHz}, \mathrm{CDCl}_{3}\right)$

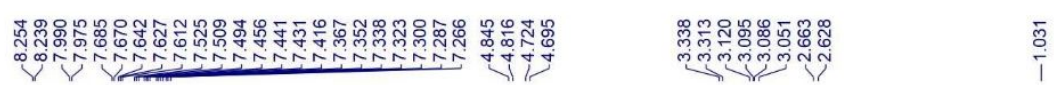

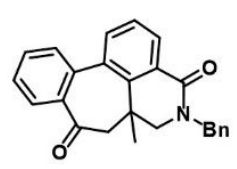

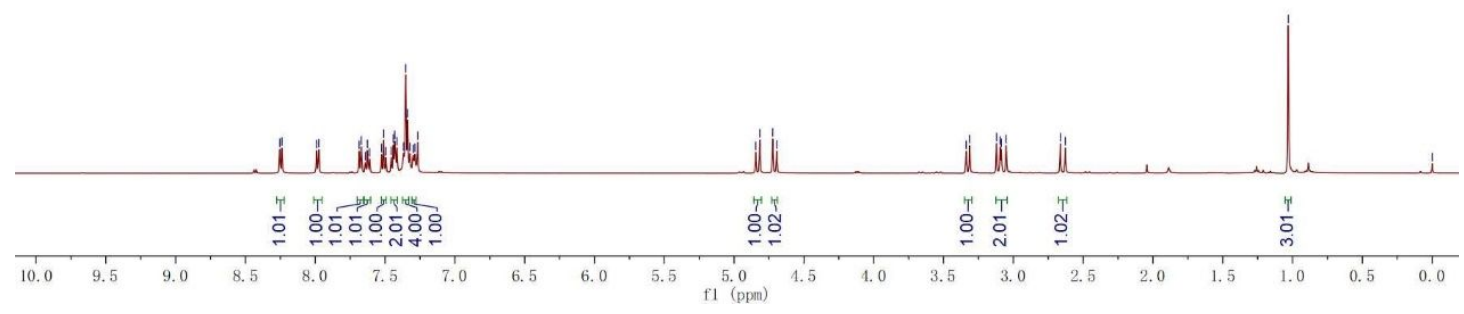

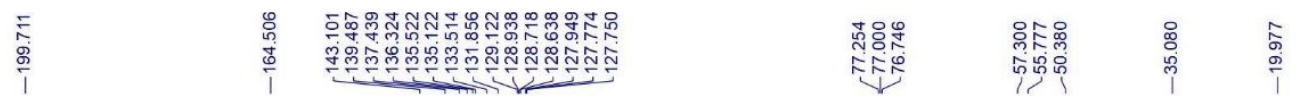
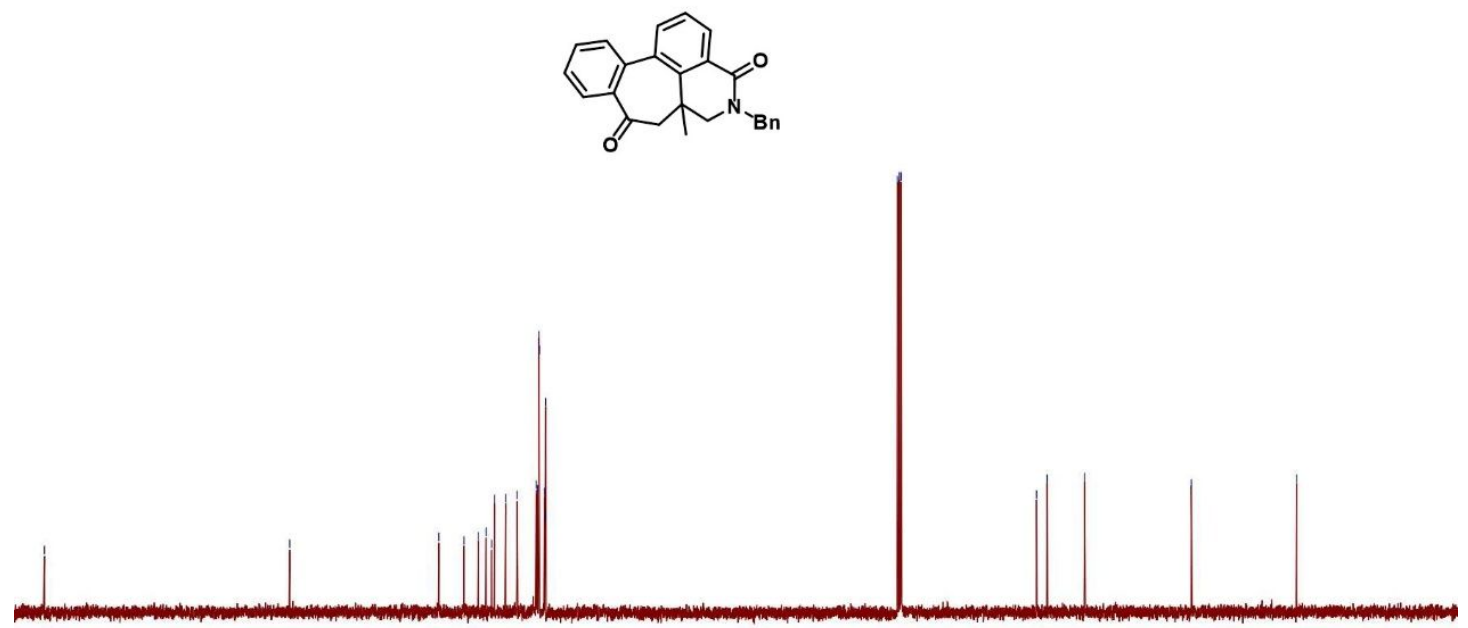

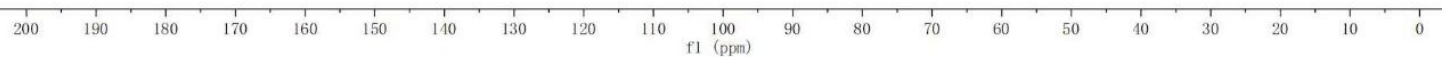


${ }^{1} \mathrm{H}$ NMR of $\mathbf{3 a b}\left(500 \mathrm{MHz}, \mathrm{CDCl}_{3}\right)$ and ${ }^{13} \mathrm{C} \mathrm{NMR} \mathrm{of} \mathbf{3 a b}\left(125 \mathrm{MHz}, \mathrm{CDCl}_{3}\right)$

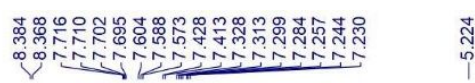

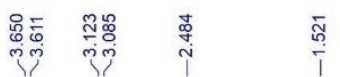


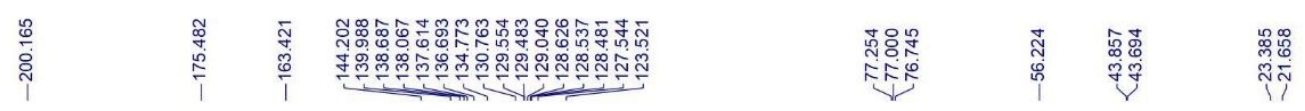
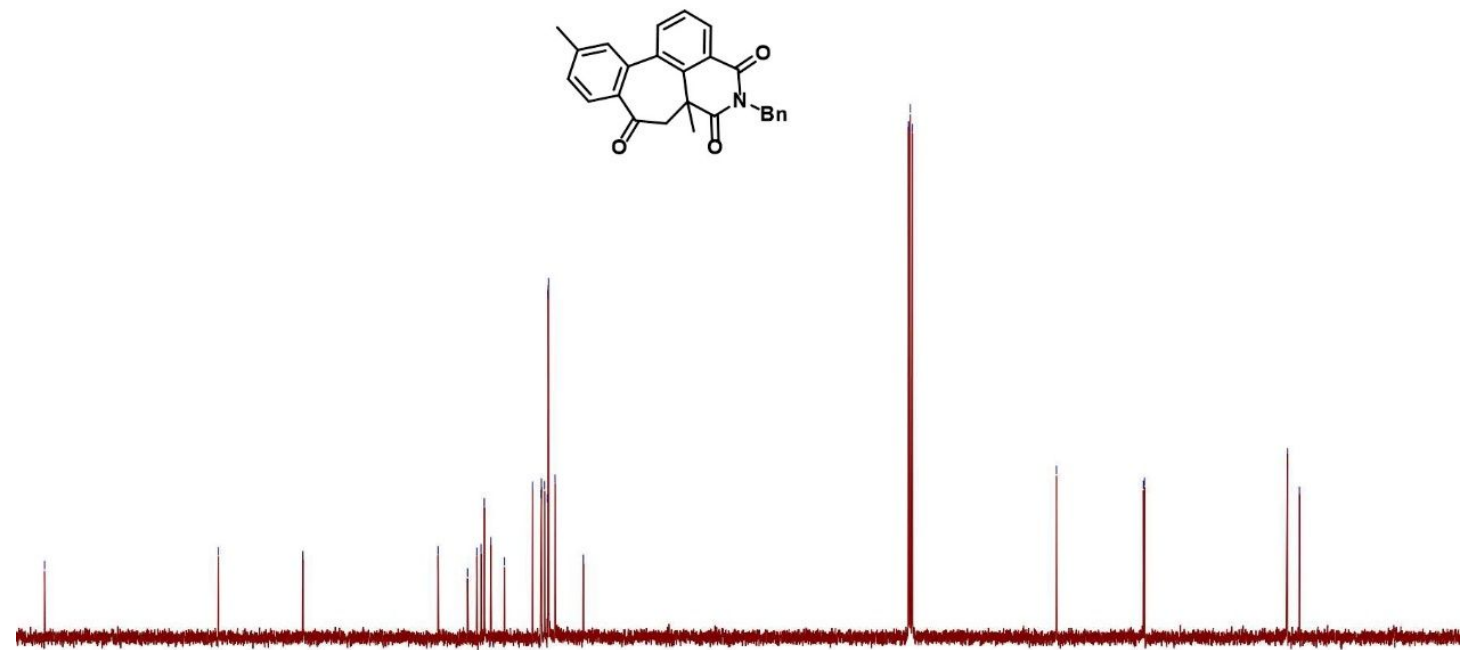

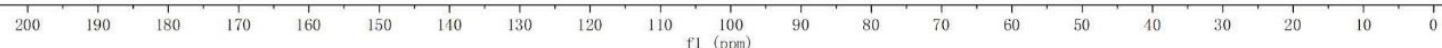


${ }^{1} \mathrm{H}$ NMR of $\mathbf{3 a c}\left(500 \mathrm{MHz}, \mathrm{CDCl}_{3}\right)$ and ${ }^{13} \mathrm{C}$ NMR of $\mathbf{3 a c}\left(125 \mathrm{MHz}, \mathrm{CDCl}_{3}\right)$

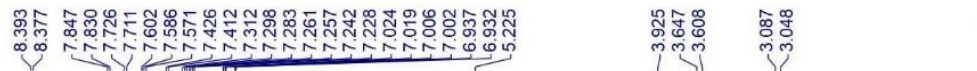

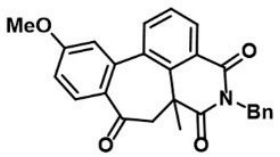

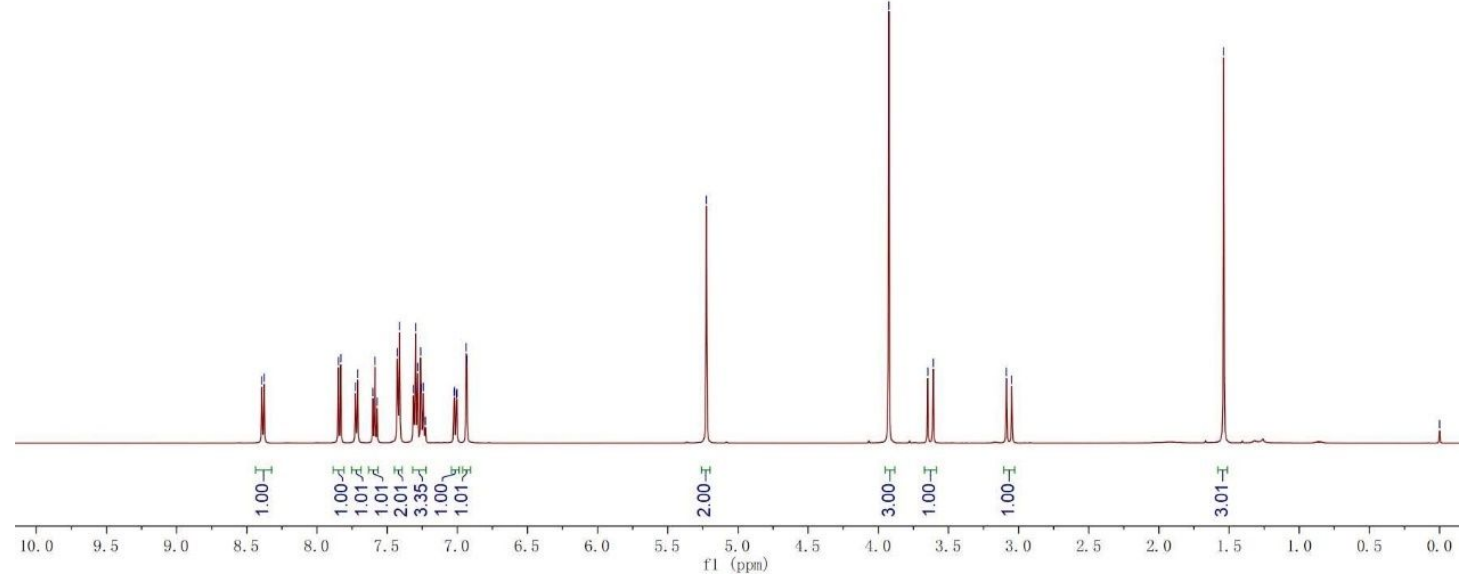

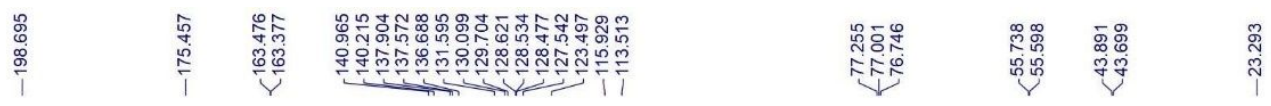




${ }^{1} \mathrm{H} \mathrm{NMR}$ of $3 \mathbf{a d}\left(500 \mathrm{MHz}, \mathrm{CDCl}_{3}\right)$ and ${ }^{13} \mathrm{C} \mathrm{NMR}$ of $\mathbf{3 a d}\left(125 \mathrm{MHz}, \mathrm{CDCl}_{3}\right)$

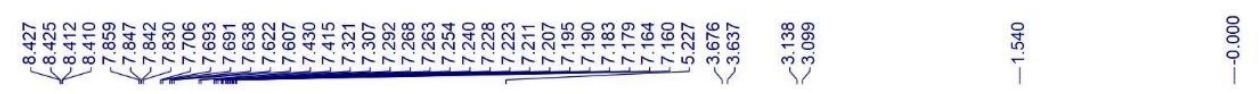
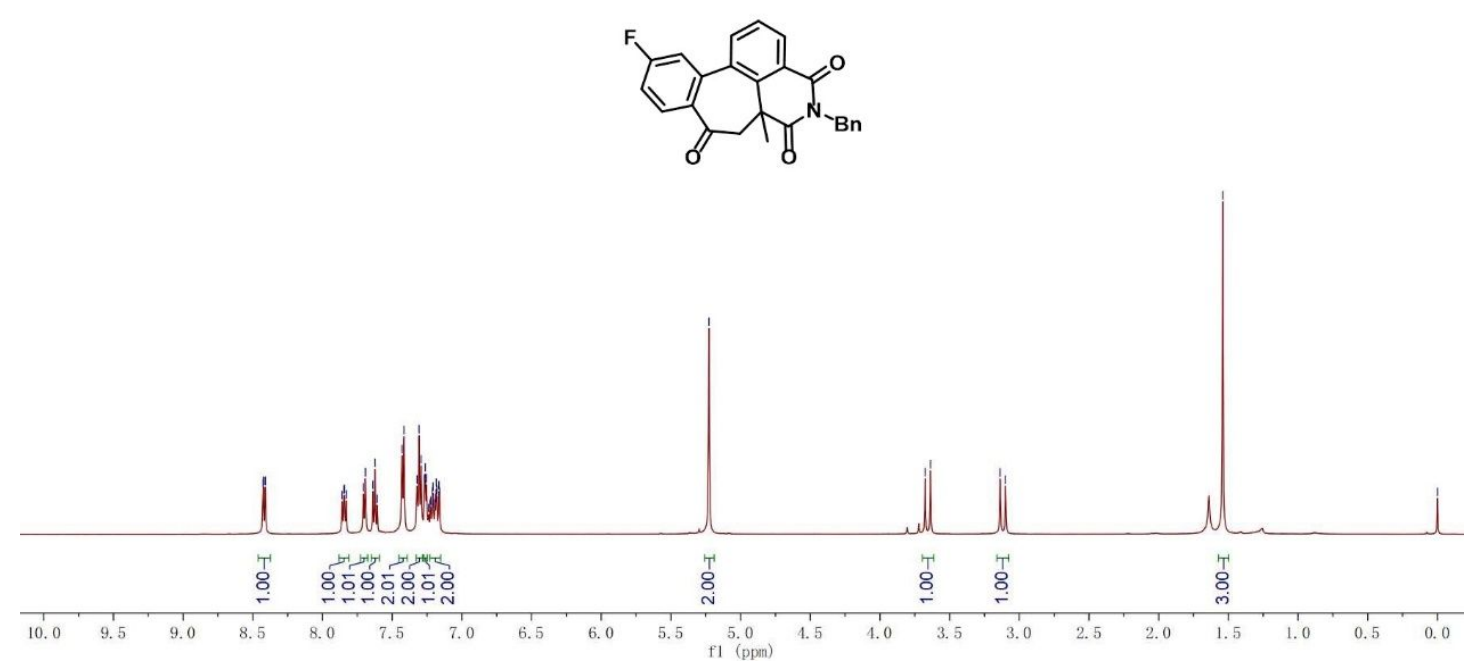

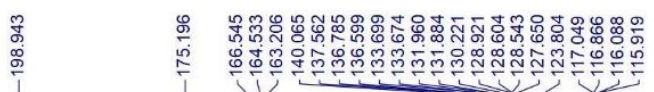

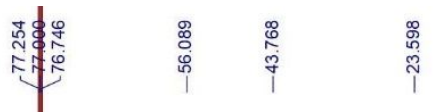





${ }^{1} \mathrm{H}$ NMR of 3ae $\left(500 \mathrm{MHz}, \mathrm{CDCl}_{3}\right)$ and ${ }^{13} \mathrm{C} \mathrm{NMR}$ of 3ae $\left(125 \mathrm{MHz}, \mathrm{CDCl}_{3}\right)$

策早

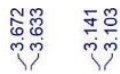
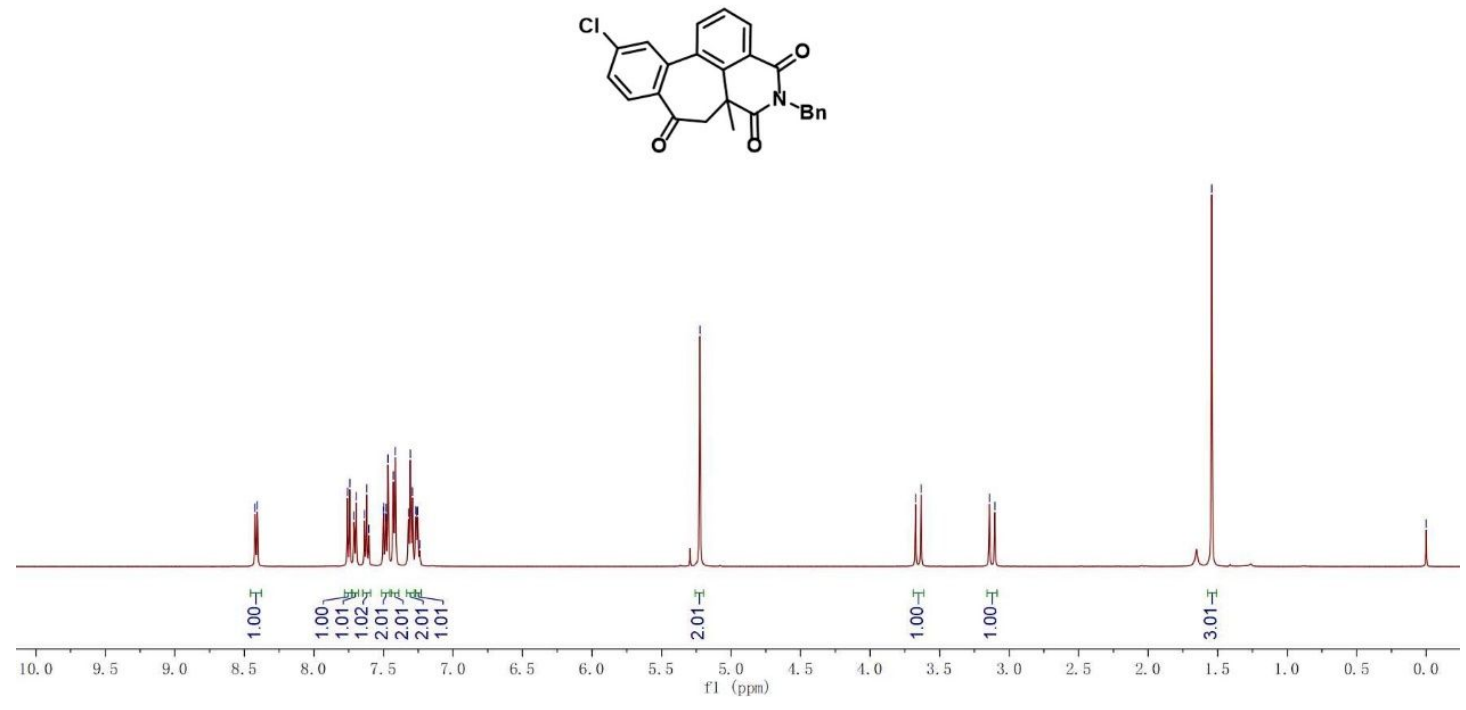



${ }^{1} \mathrm{H}$ NMR of $\mathbf{3 a f}\left(500 \mathrm{MHz}, \mathrm{CDCl}_{3}\right)$ and ${ }^{13} \mathrm{C} \mathrm{NMR}$ of $\mathbf{3 a f}\left(125 \mathrm{MHz}, \mathrm{CDCl}_{3}\right)$

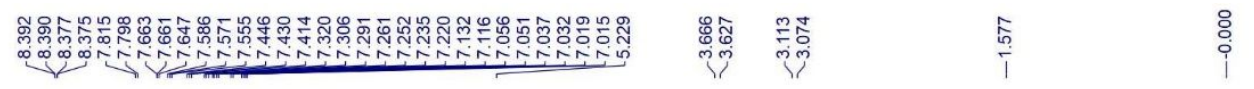<smiles>CN1C(=O)C2CC(=O)C3(C)c4c(cccc4C2C1=O)C(=O)[NH+]3C</smiles>

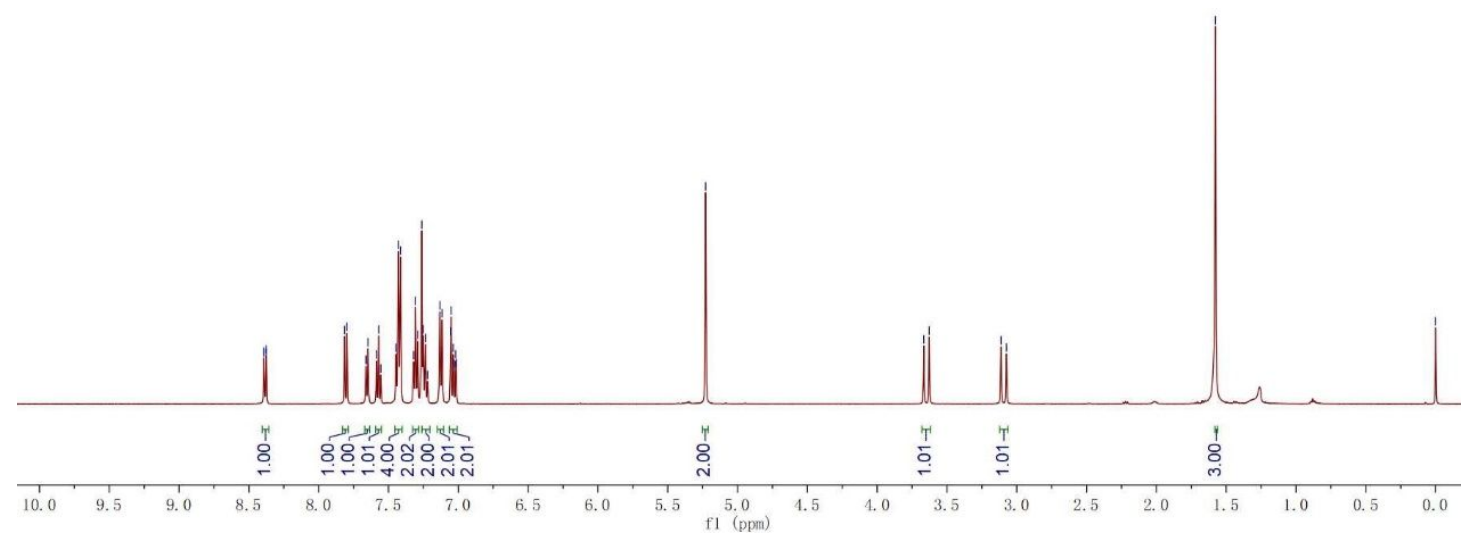

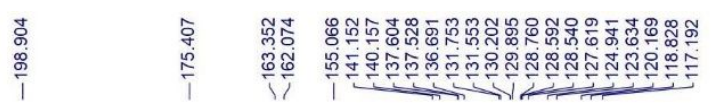

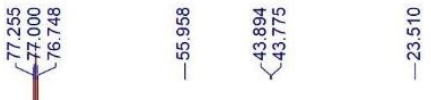
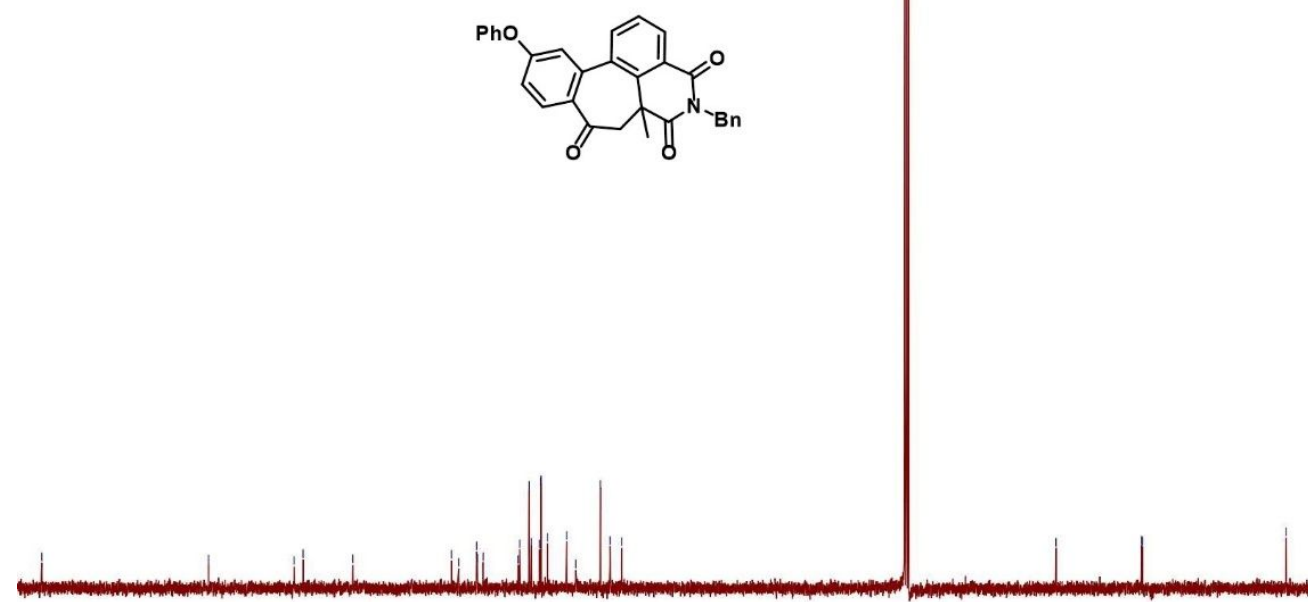

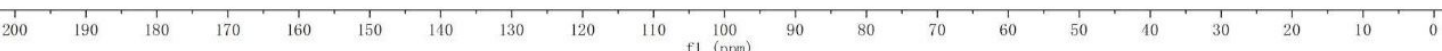


${ }^{1} \mathrm{H}$ NMR of 3ag (500 MHz, $\mathrm{CDCl}_{3}$ ) and ${ }^{13} \mathrm{C}$ NMR of $3 \mathbf{a g}\left(125 \mathrm{MHz}, \mathrm{CDCl}_{3}\right)$


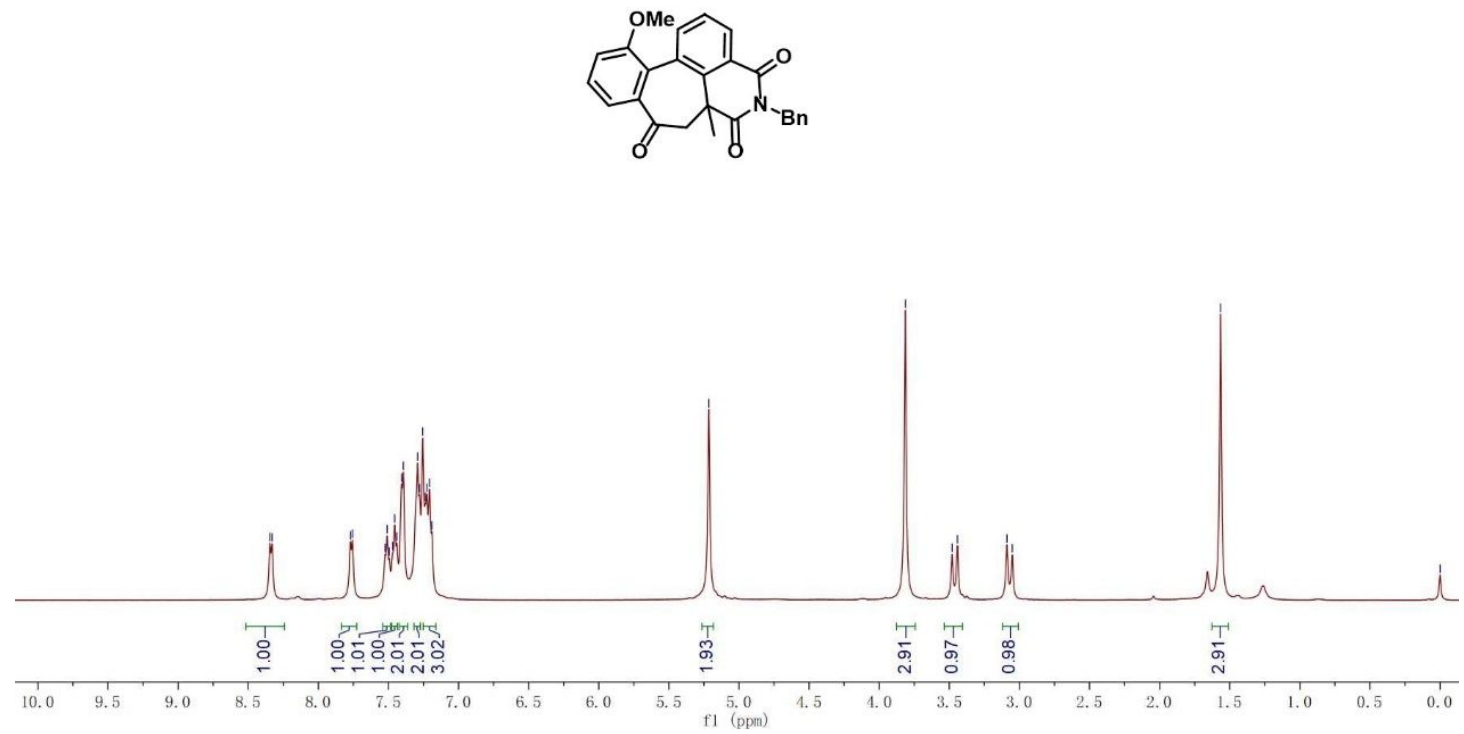


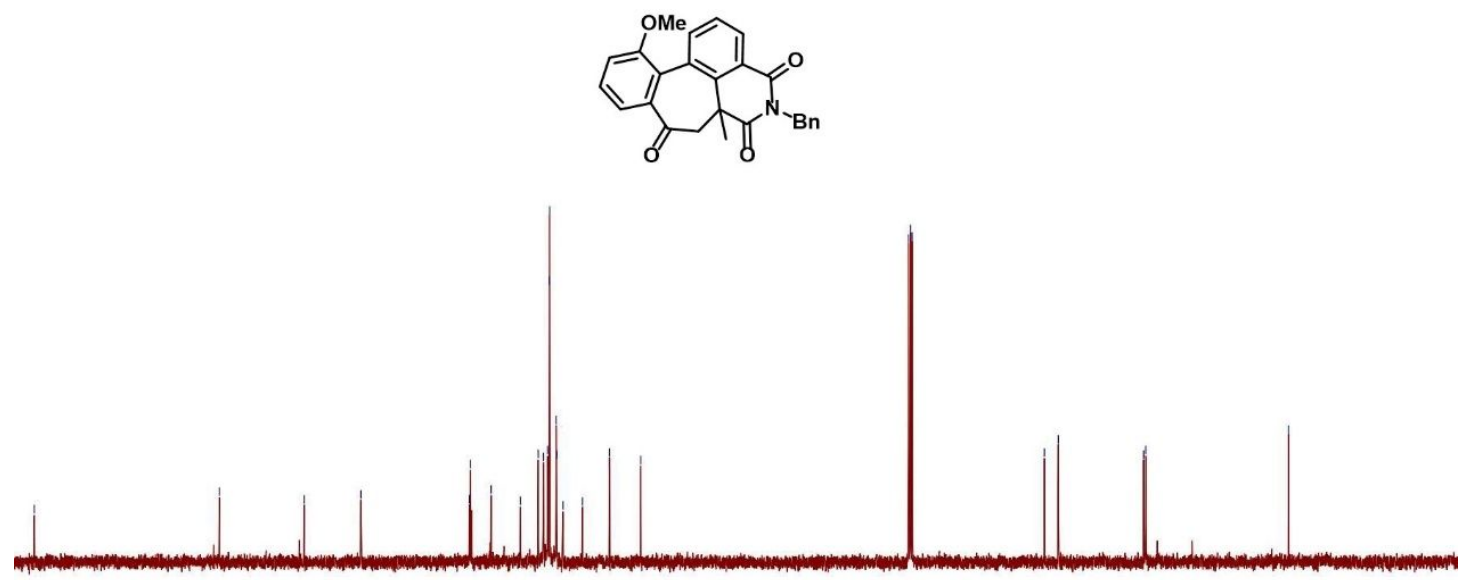

$\begin{array}{lllllllllll}200 & 190 & 180 & 170 & 160 & 150 & 140 & 130 & 120 & 110 & 100\end{array}$ 
${ }^{1} \mathrm{H}$ NMR of $\mathbf{3 a h}\left(500 \mathrm{MHz}, \mathrm{DMSO}-\mathrm{d}_{6}\right)$ and ${ }^{13} \mathrm{C}$ NMR of $\mathbf{3 a h}\left(125 \mathrm{MHz}, \mathrm{DMSO}-\mathrm{d}_{6}\right)$

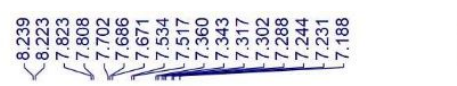
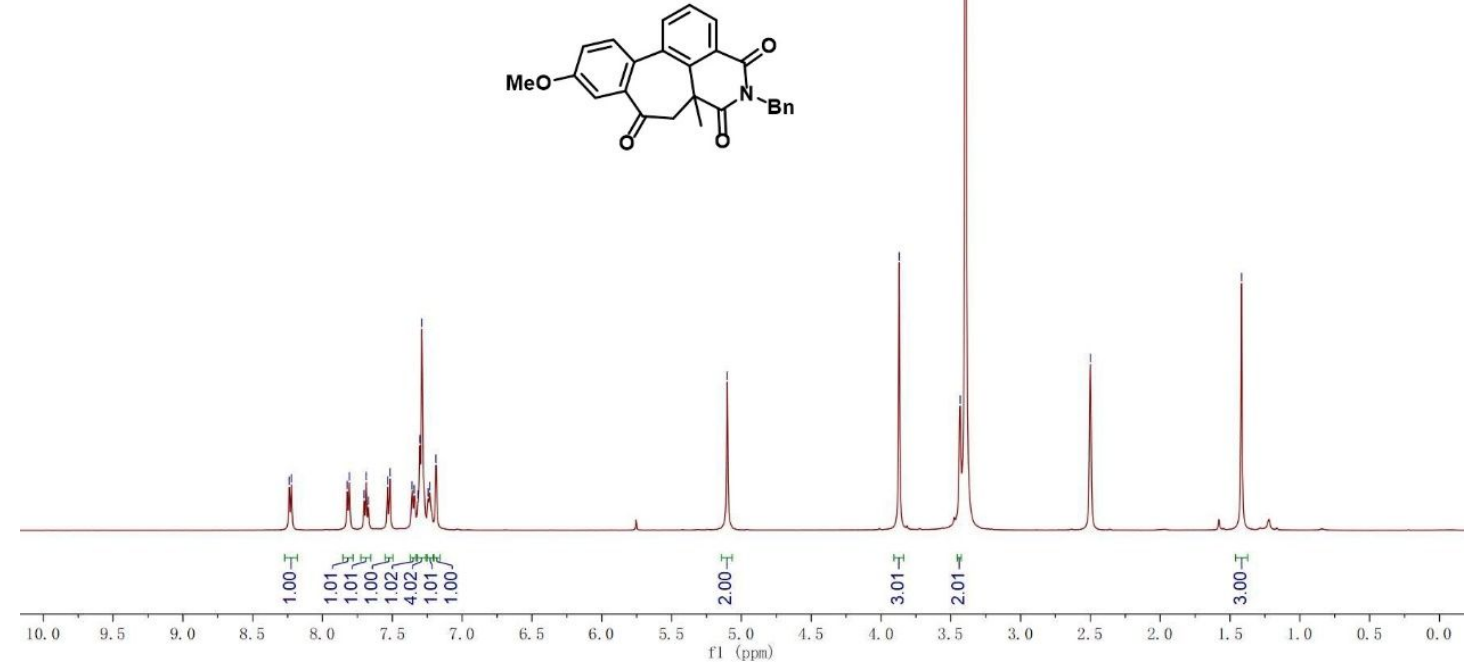

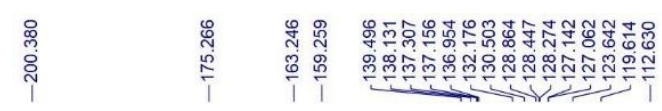
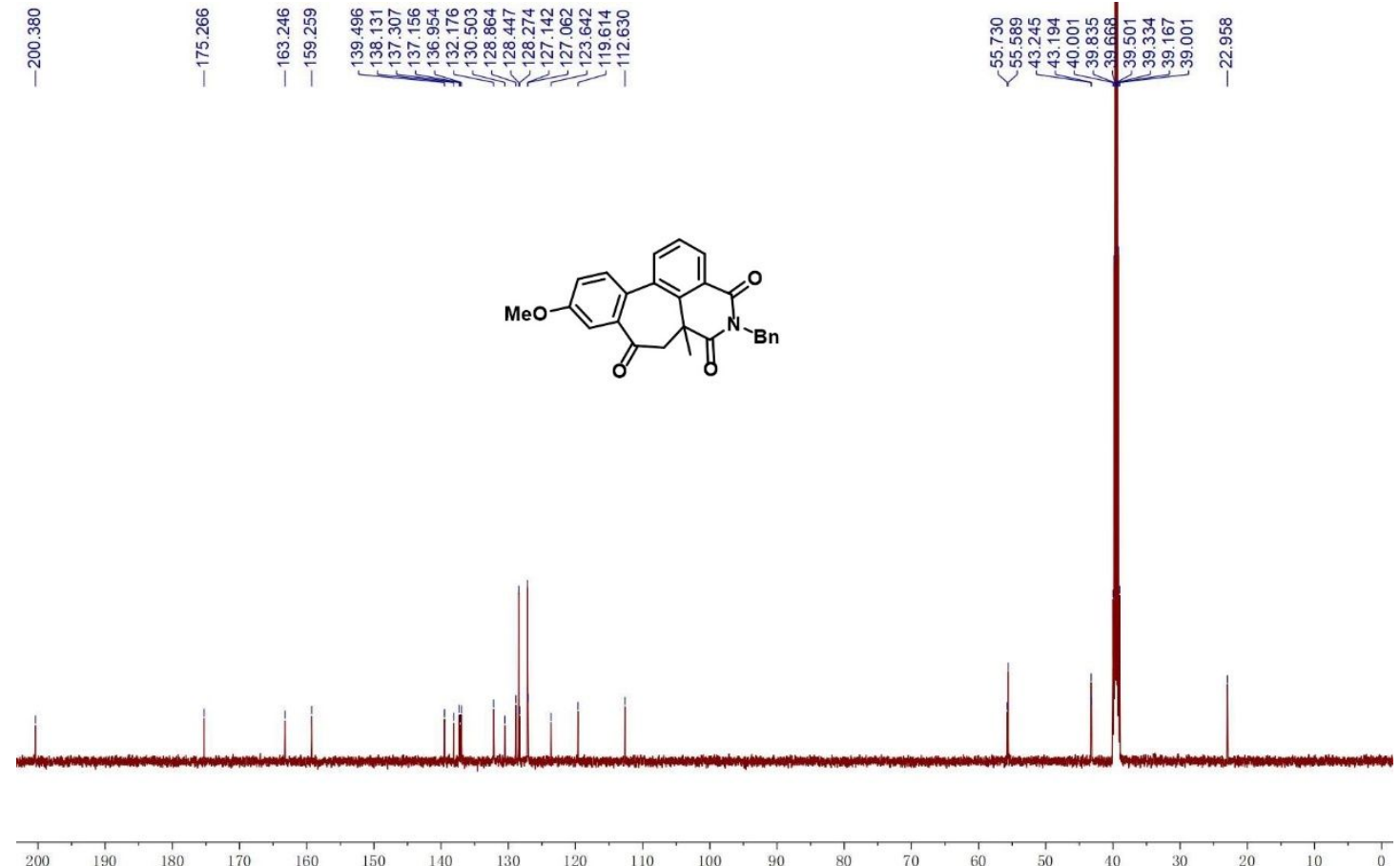

$150 \quad 140$

$110 \stackrel{100}{\mathrm{fl}} \stackrel{(\mathrm{ppm})}{\mathrm{g}}$ 
${ }^{1} \mathrm{H}$ NMR of 3ai (500 MHz, $\mathrm{CDCl}_{3}$ ) and ${ }^{13} \mathrm{C}$ NMR of 3ai ( $\left.125 \mathrm{MHz}, \mathrm{CDCl}_{3}\right)$

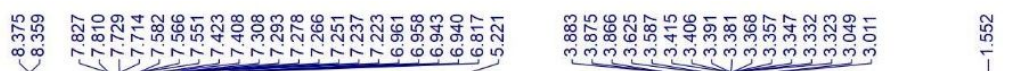
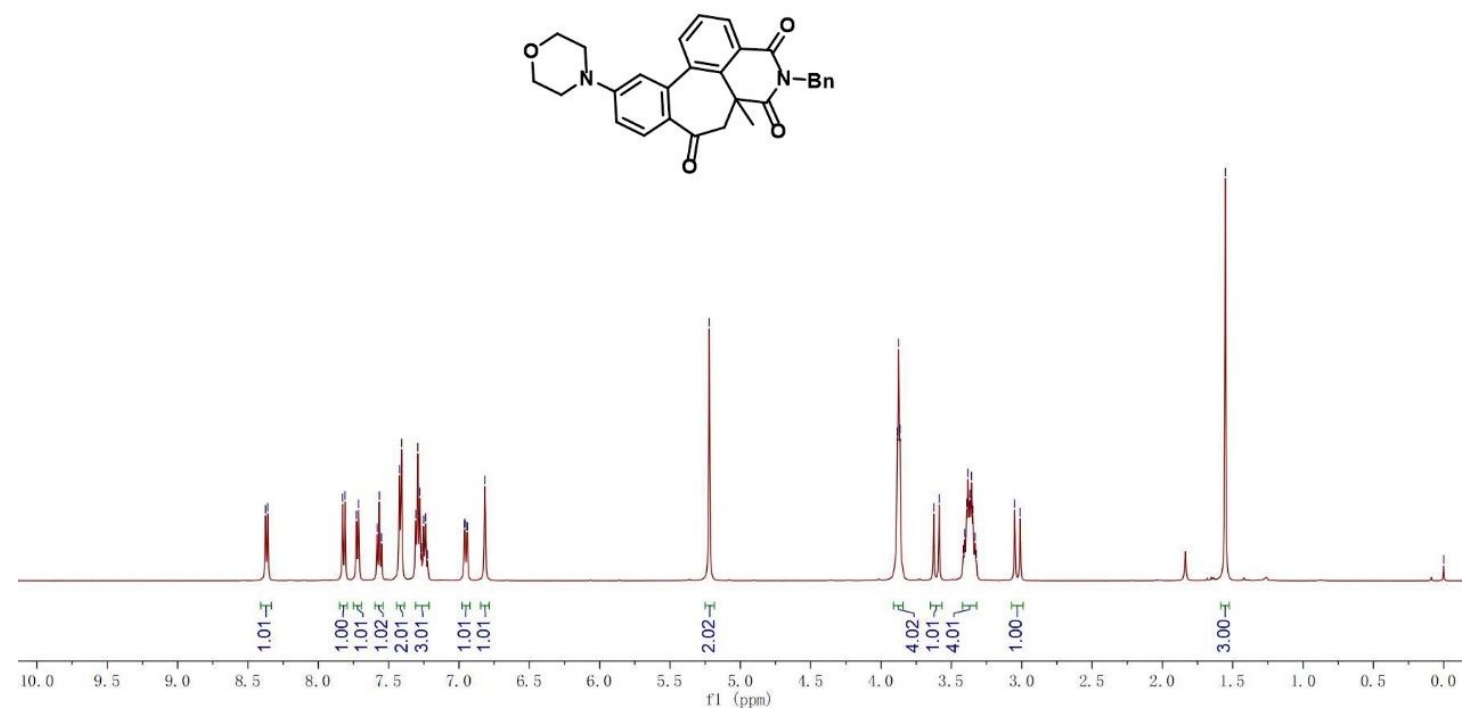

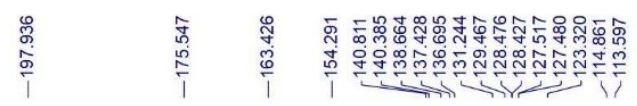

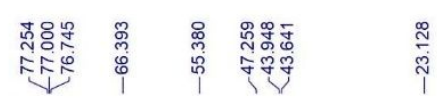


$200 \quad 190 \quad 180 \quad 170 \quad 1$




${ }^{1} \mathrm{H}$ NMR of 3aj (500 MHz, $\mathrm{CDCl}_{3}$ ) and ${ }^{13} \mathrm{C}$ NMR of 3aj (125 MHz, $\left.\mathrm{CDCl}_{3}\right)$

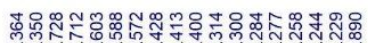

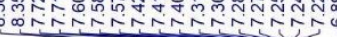
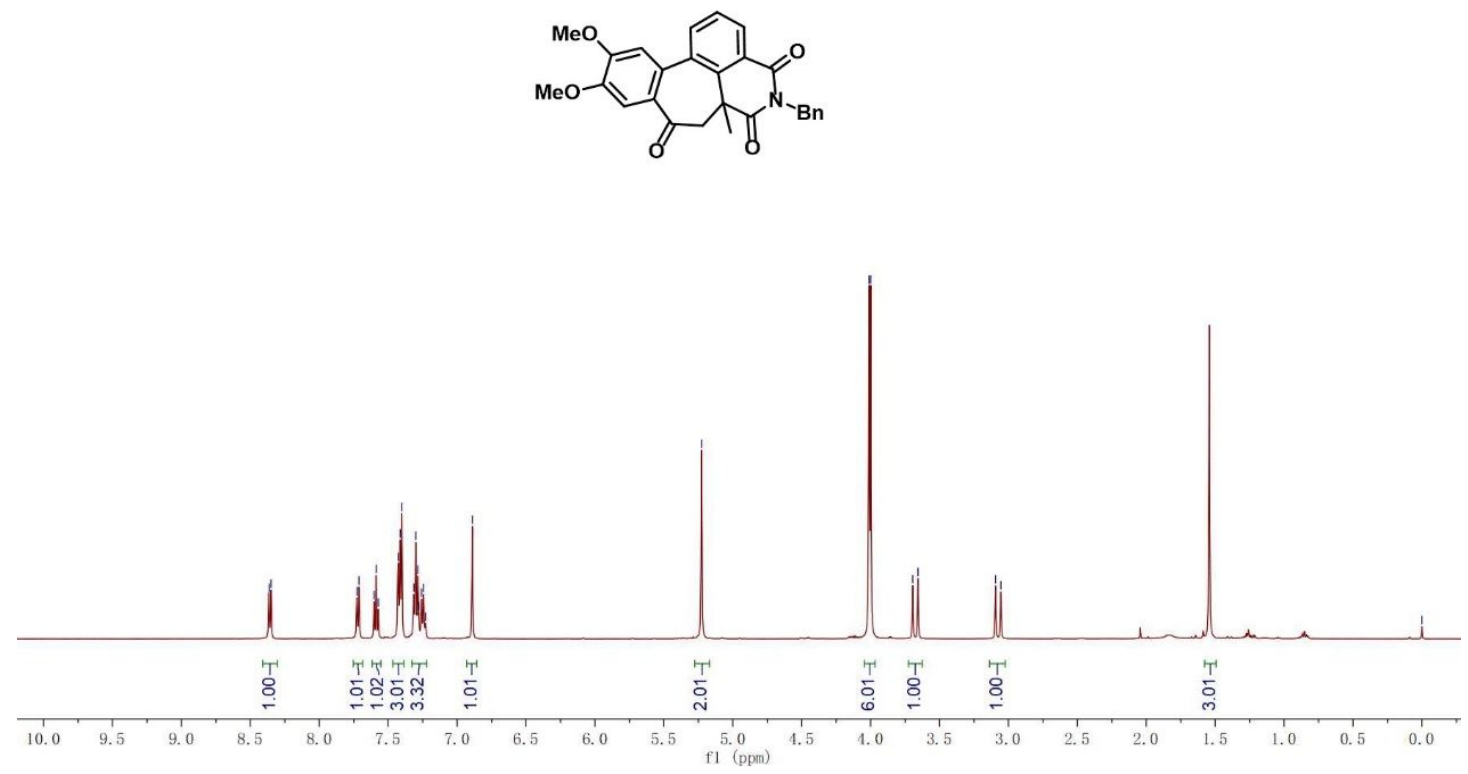

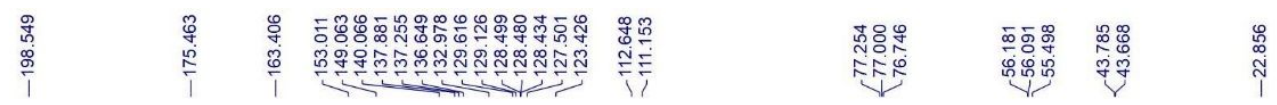


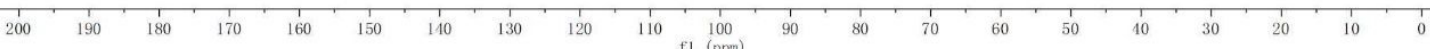


${ }^{1} \mathrm{H}$ NMR of $3 \mathbf{a k}\left(500 \mathrm{MHz}, \mathrm{CDCl}_{3}\right)$ and ${ }^{13} \mathrm{C} \mathrm{NMR}$ of $\mathbf{3 a k}\left(125 \mathrm{MHz}, \mathrm{CDCl}_{3}\right)$

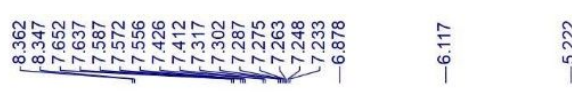

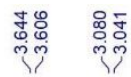

$\stackrel{\hat{n}}{i}$
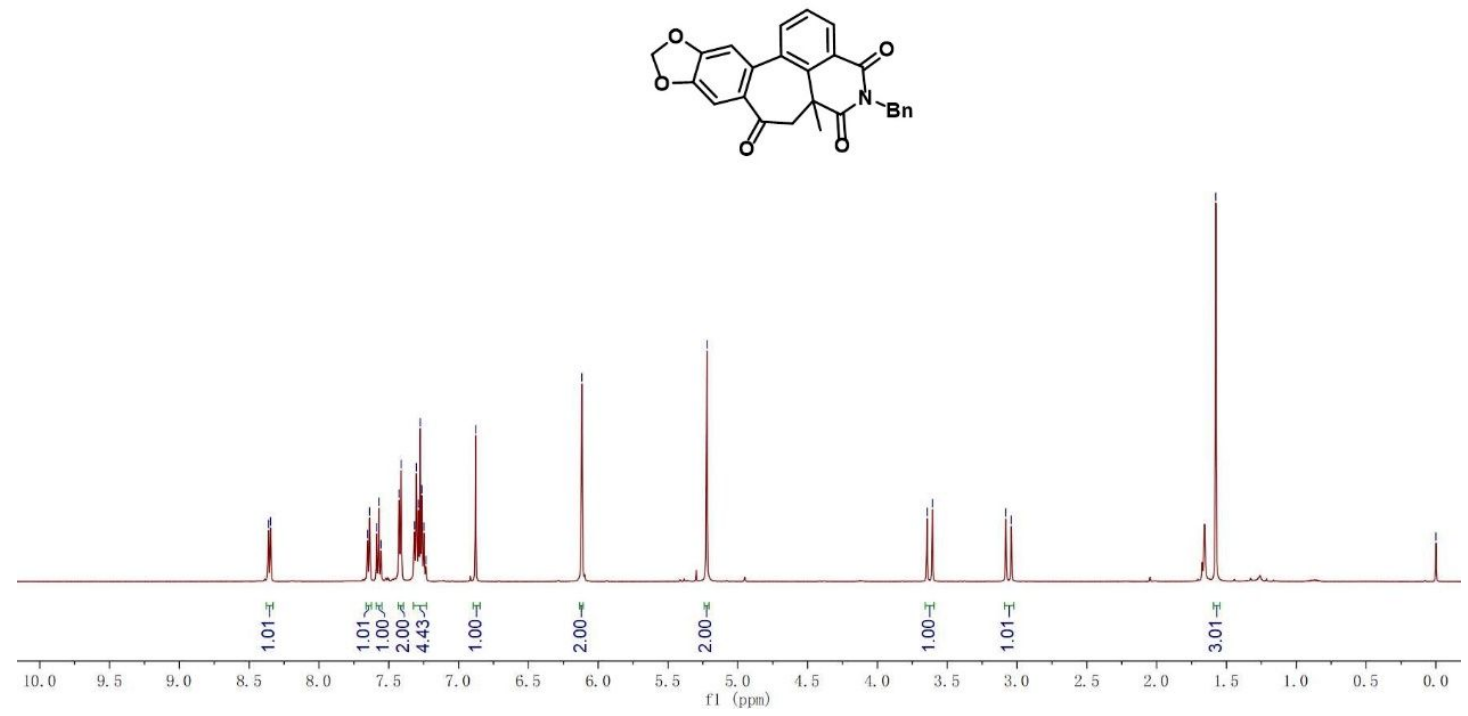



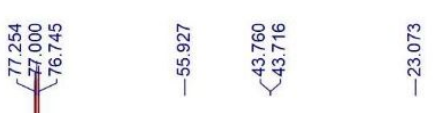
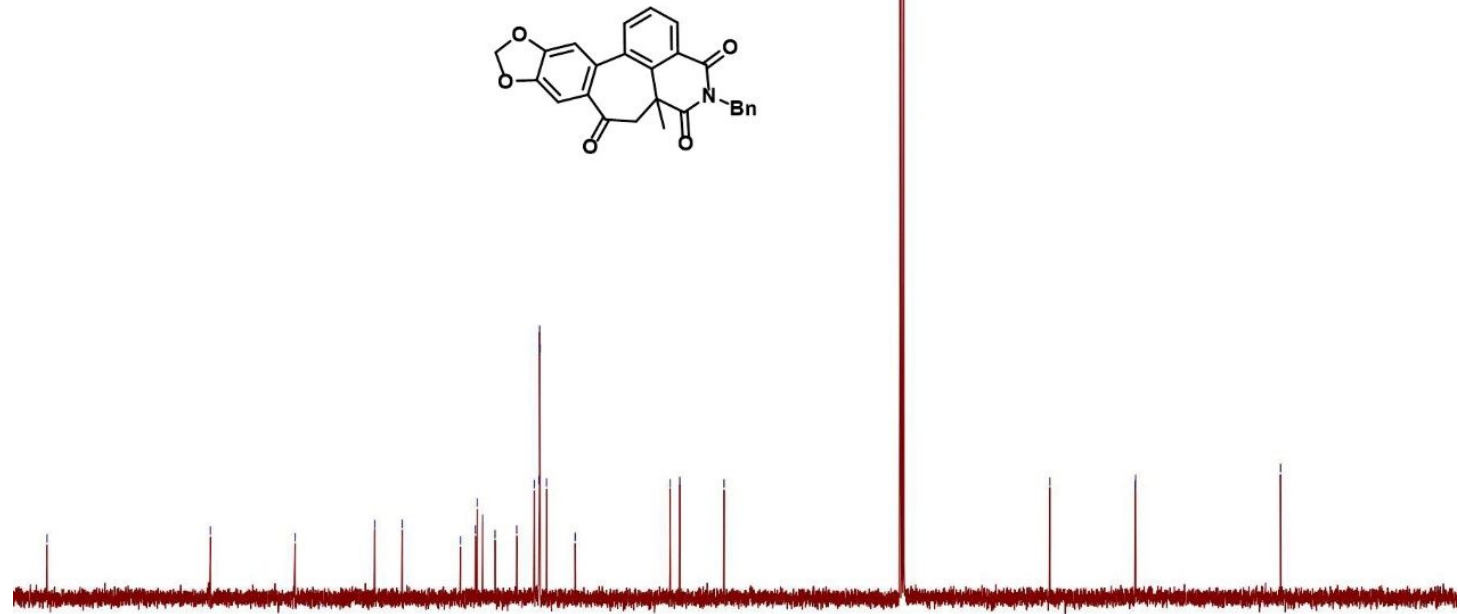

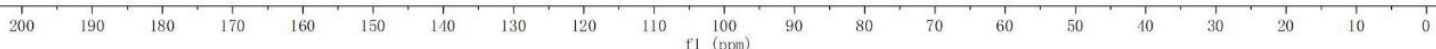


${ }^{1} \mathrm{H}$ NMR of 3al $\left(500 \mathrm{MHz}, \mathrm{CDCl}_{3}\right)$ and ${ }^{13} \mathrm{C}$ NMR of 3al $\left(125 \mathrm{MHz}, \mathrm{CDCl}_{3}\right)$

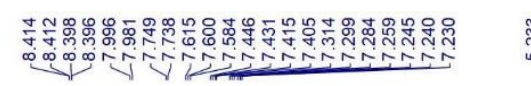

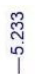

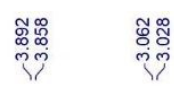
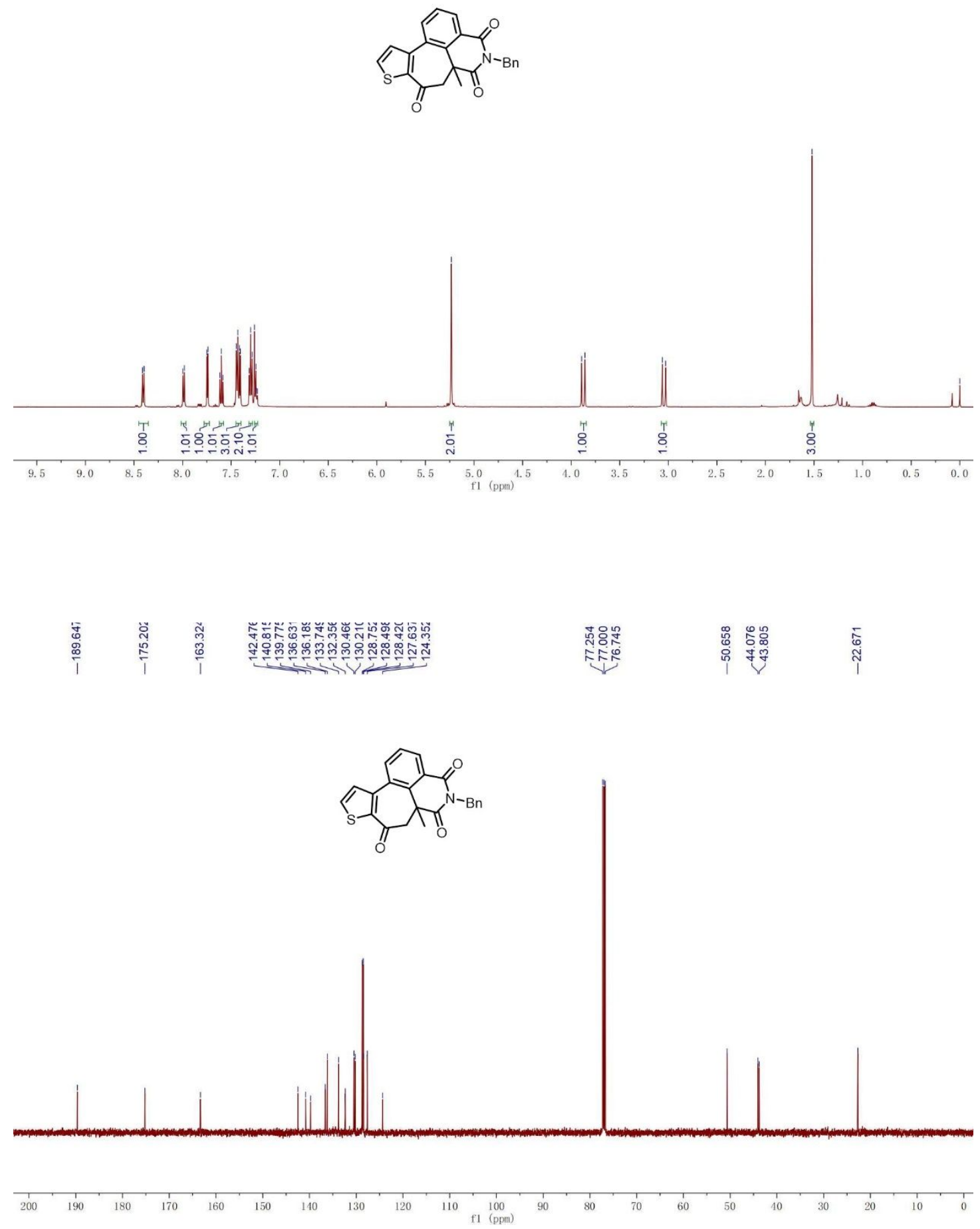
${ }^{1} \mathrm{H} \mathrm{NMR}$ of $5 \mathbf{a a}\left(500 \mathrm{MHz}, \mathrm{CDCl}_{3}\right)$ and ${ }^{13} \mathrm{C} \mathrm{NMR}$ of $\mathbf{5 a a}\left(125 \mathrm{MHz}, \mathrm{CDCl}_{3}\right)$







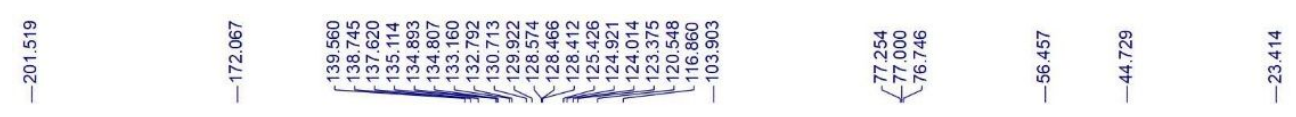
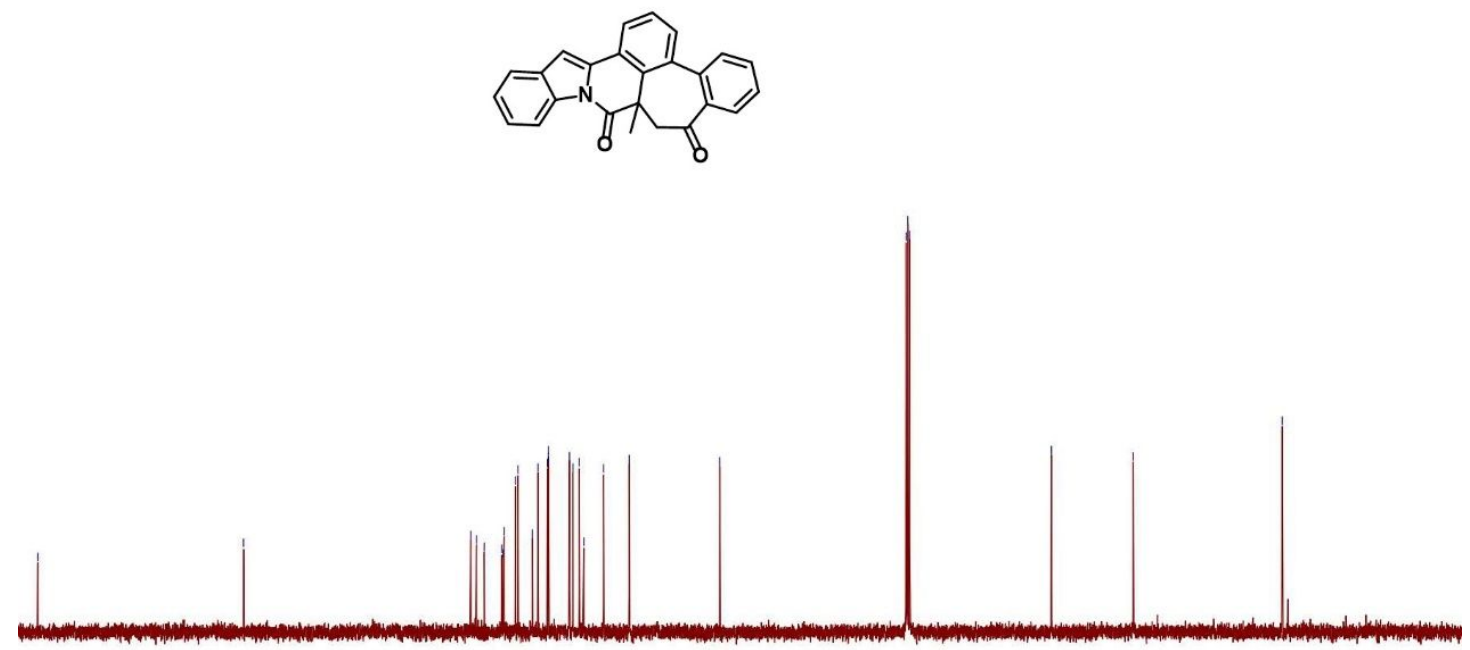

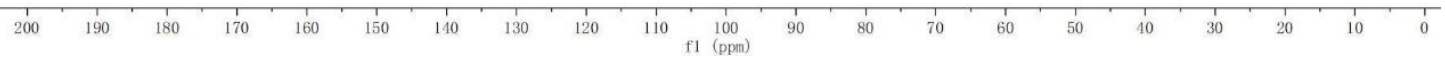


${ }^{1} \mathrm{H}$ NMR of $5 \mathbf{b a}\left(500 \mathrm{MHz}, \mathrm{CDCl}_{3}\right)$ and ${ }^{13} \mathrm{C} \mathrm{NMR}$ of $\mathbf{5 b a}\left(125 \mathrm{MHz}, \mathrm{CDCl}_{3}\right)$

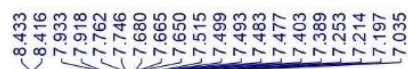

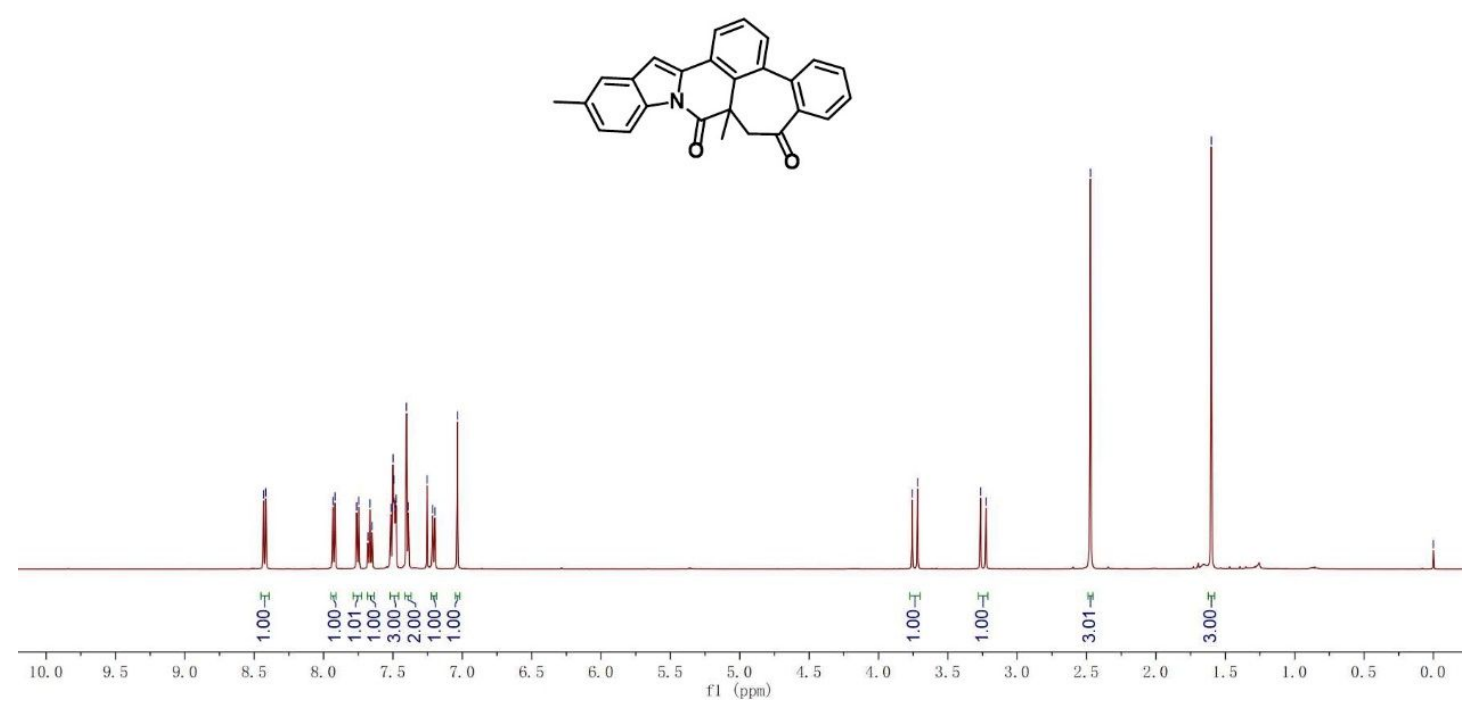


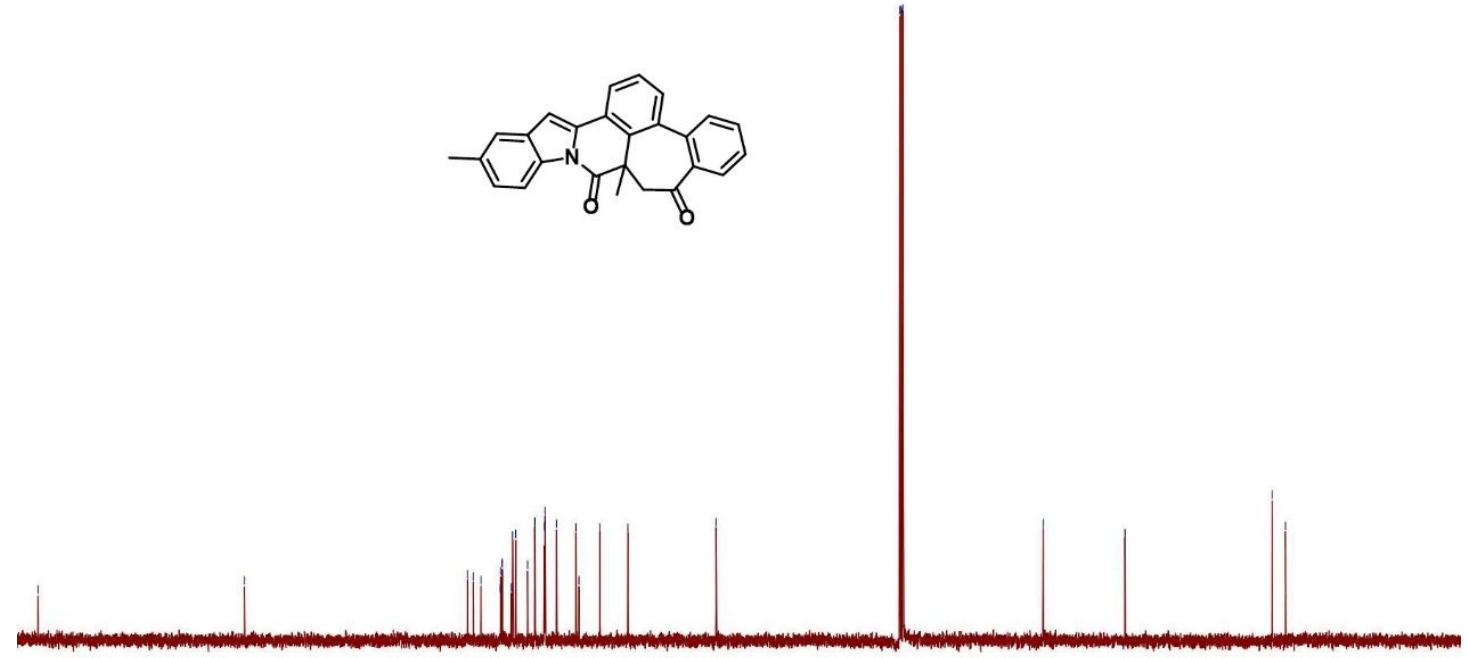




${ }^{1} \mathrm{H}$ NMR of $\mathbf{5} \mathbf{c a}\left(500 \mathrm{MHz}, \mathrm{CDCl}_{3}\right)$ and ${ }^{13} \mathrm{C}$ NMR of $\mathbf{5} \mathbf{c a}\left(125 \mathrm{MHz}, \mathrm{CDCl}_{3}\right)$

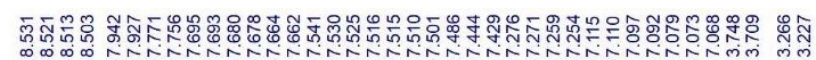

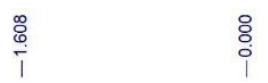

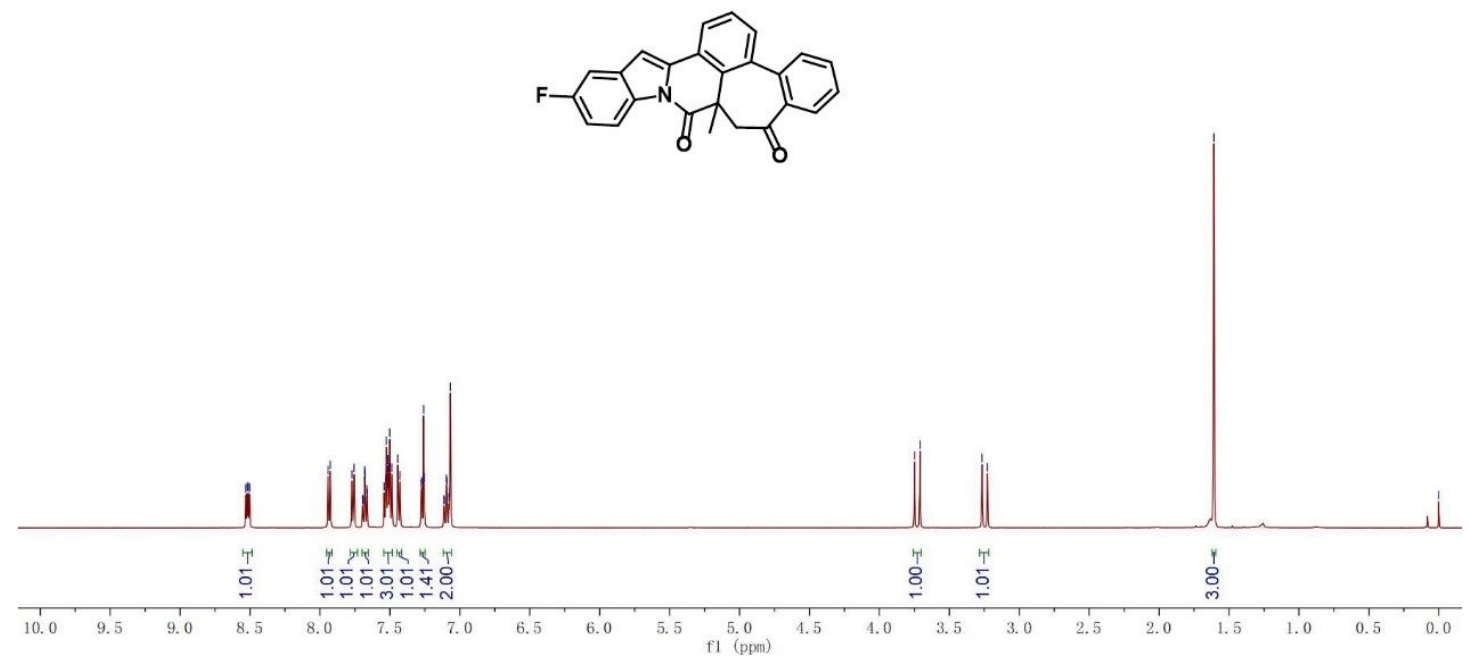

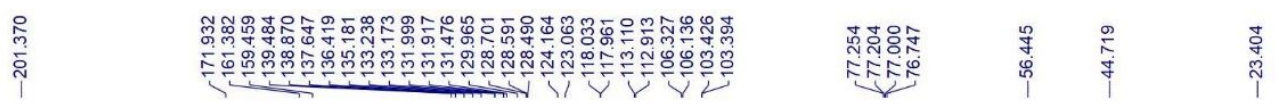
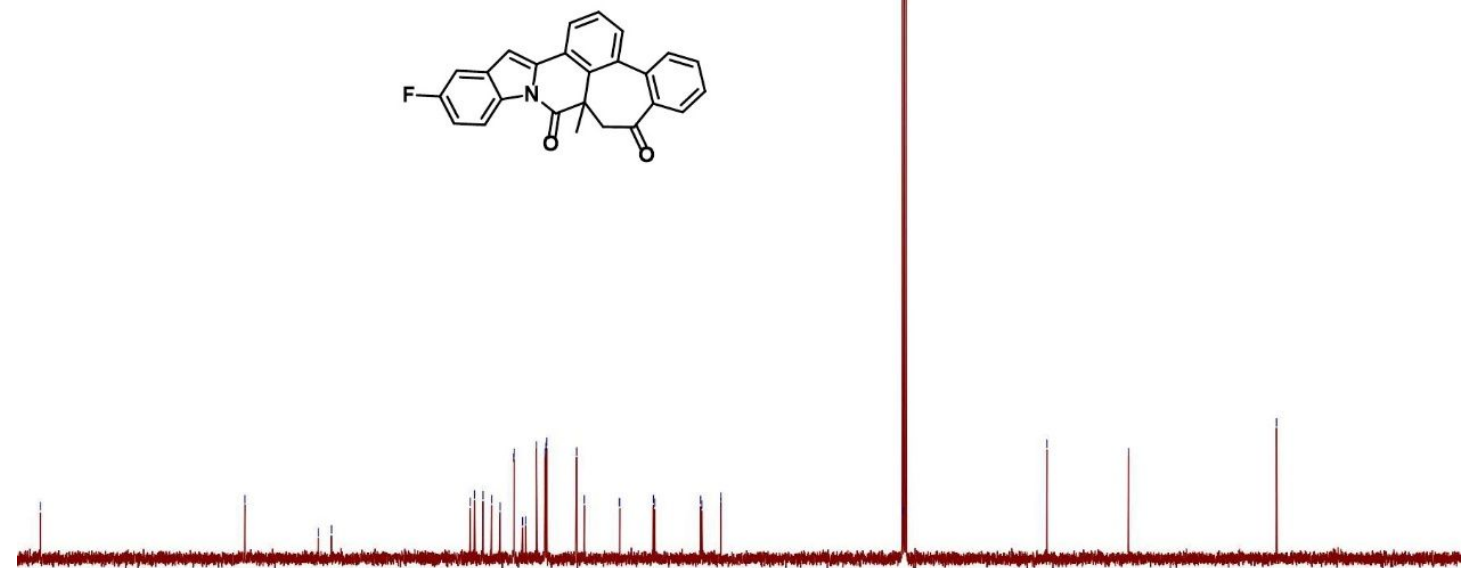

200

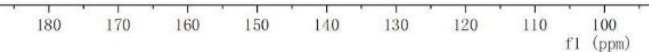


${ }^{1} \mathrm{H}$ NMR of $5 \mathbf{d a}\left(500 \mathrm{MHz}, \mathrm{CDCl}_{3}\right)$ and ${ }^{13} \mathrm{C}$ NMR of $5 \mathbf{d a}\left(125 \mathrm{MHz}, \mathrm{CDCl}_{3}\right)$

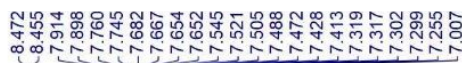

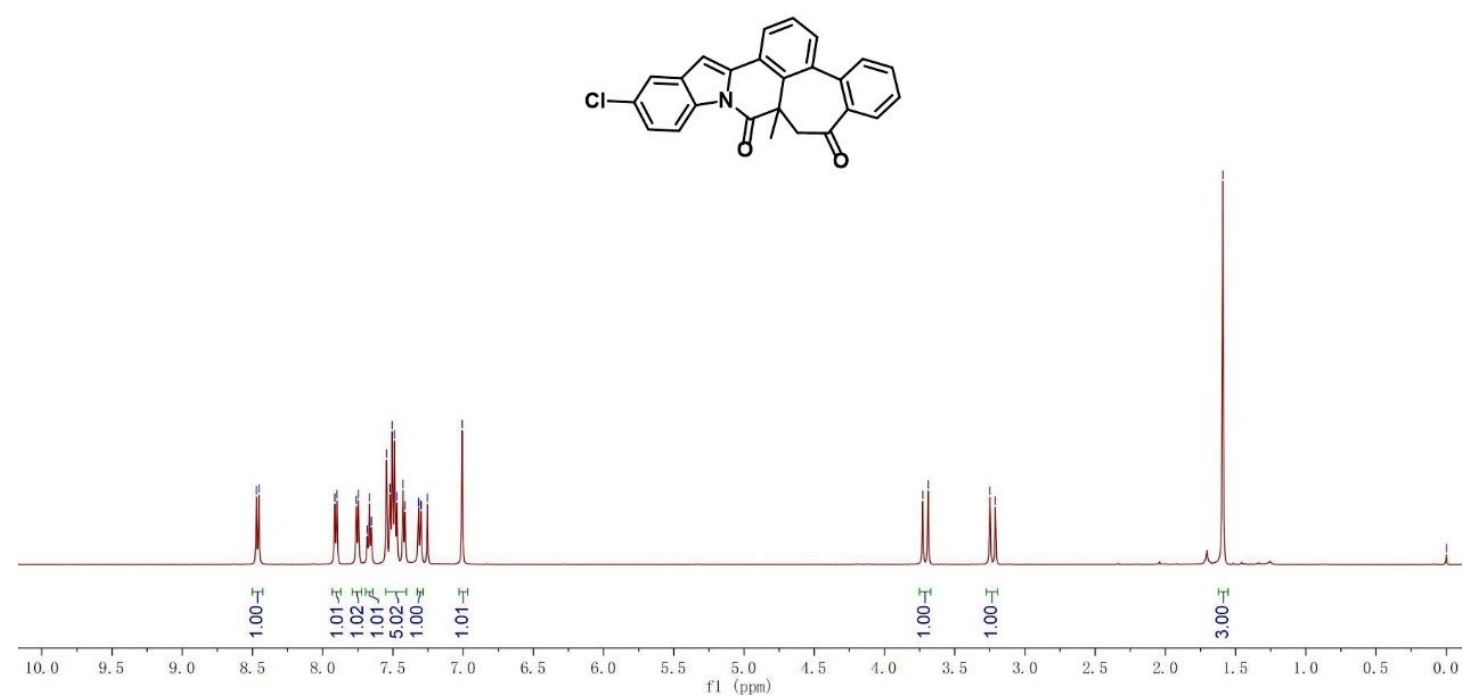



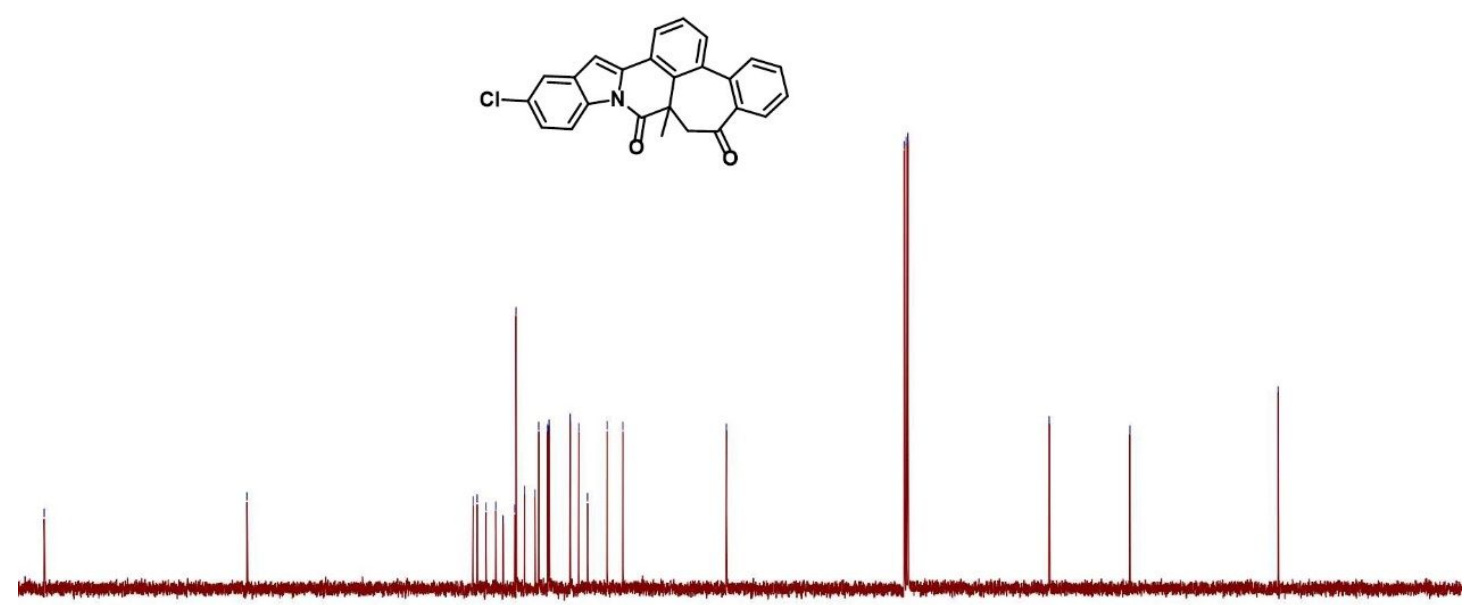

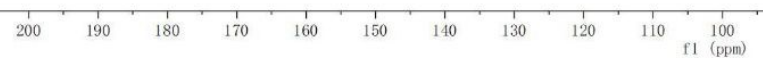


${ }^{1} \mathrm{H}$ NMR of 5 ea $\left(500 \mathrm{MHz}, \mathrm{CDCl}_{3}\right)$ and ${ }^{13} \mathrm{C} \mathrm{NMR}$ of $\mathbf{5 e a}\left(125 \mathrm{MHz}, \mathrm{CDCl}_{3}\right)$



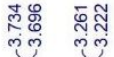

$\stackrel{\bar{\sigma}}{\square}$

$\stackrel{8}{\circ}$

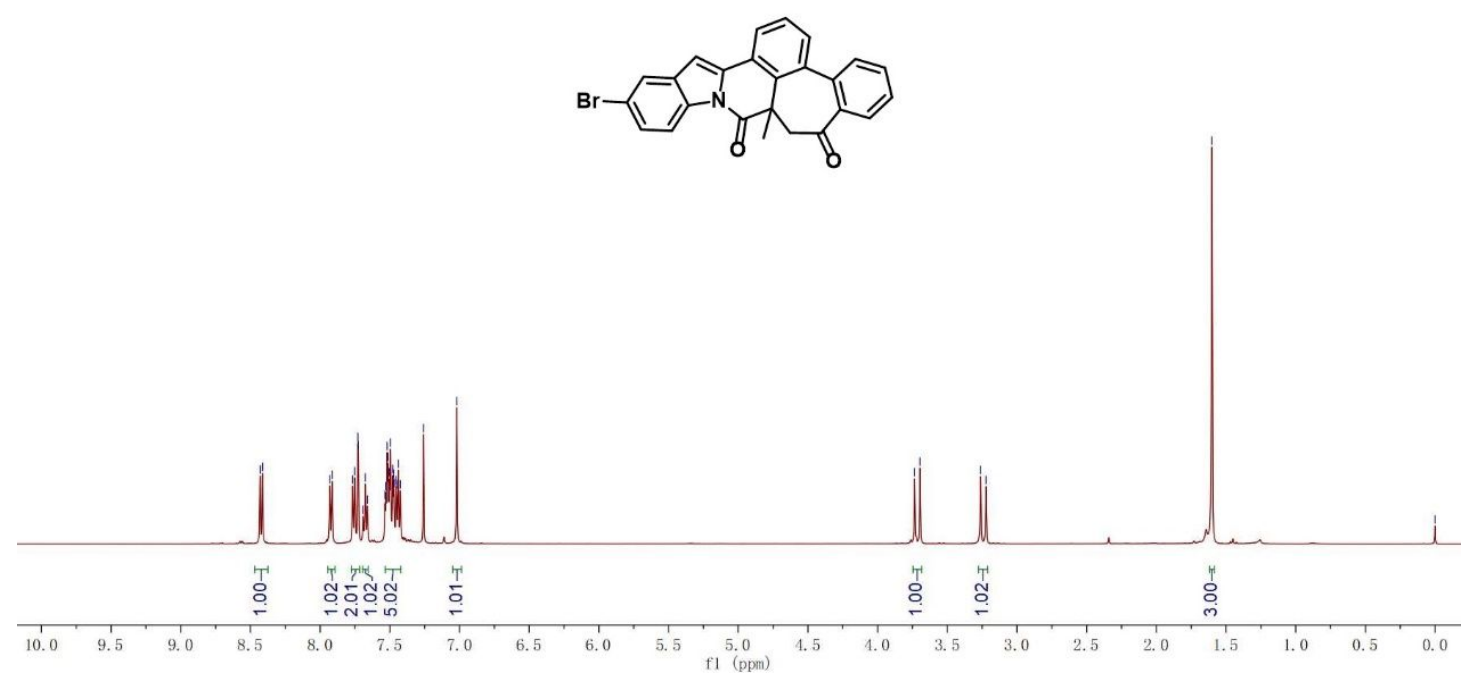

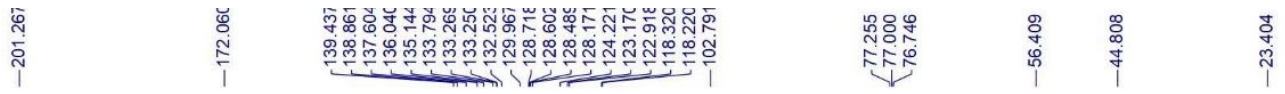

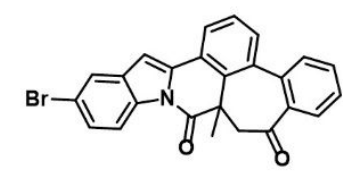

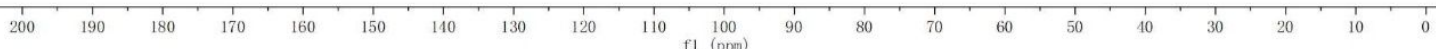


${ }^{1} \mathrm{H}$ NMR of $\mathbf{5 f a}\left(500 \mathrm{MHz}, \mathrm{CDCl}_{3}\right)$ and ${ }^{13} \mathrm{C} \mathrm{NMR}$ of $\mathbf{5 f a}\left(125 \mathrm{MHz}, \mathrm{CDCl}_{3}\right)$



(4)

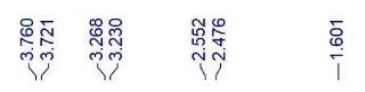
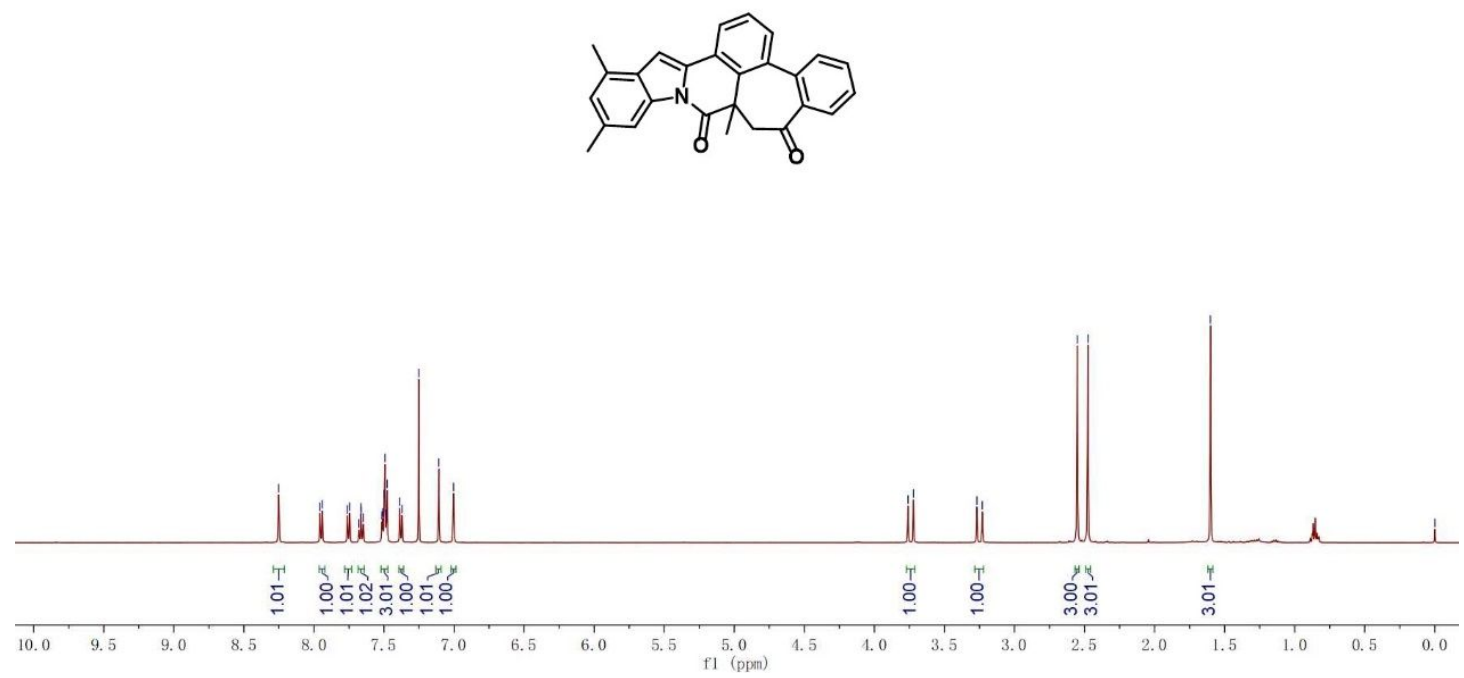

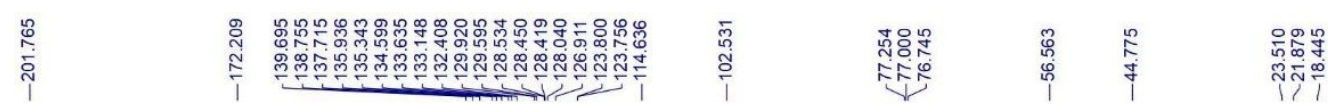
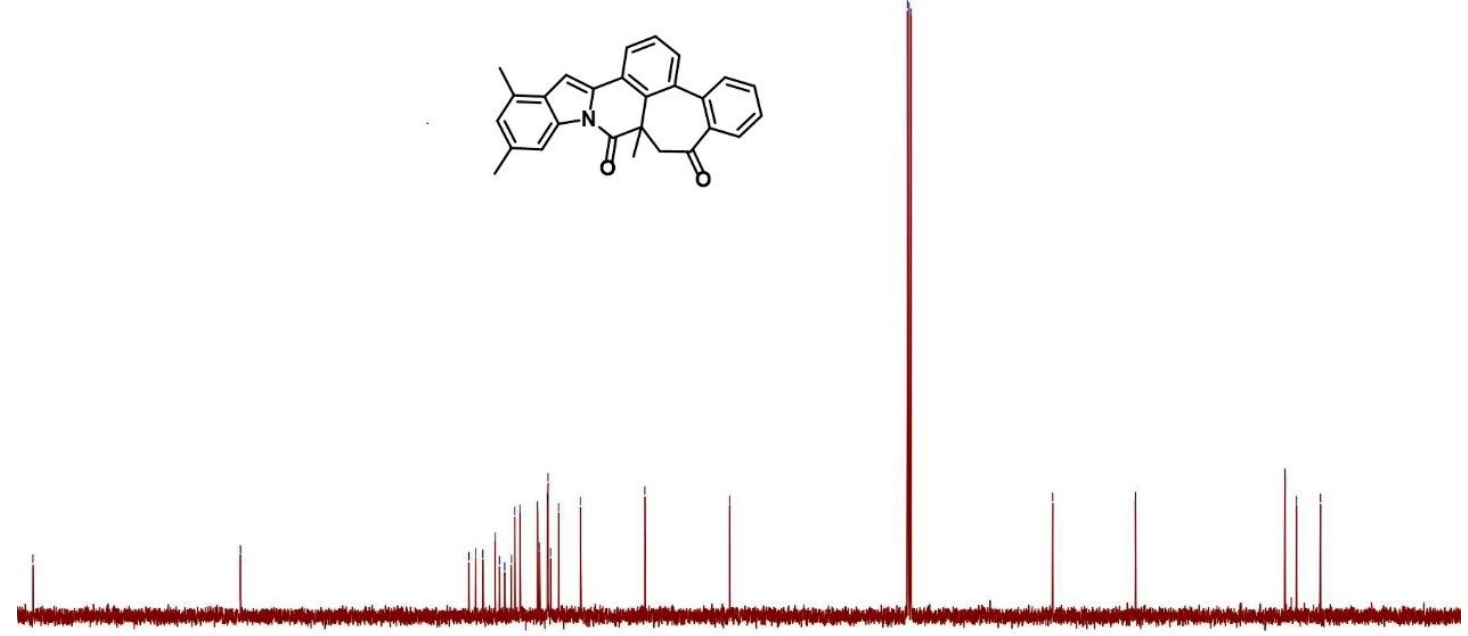

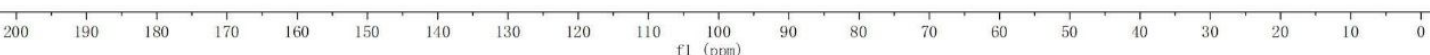


${ }^{1} \mathrm{H} \mathrm{NMR}$ of $5 \mathbf{g a}\left(500 \mathrm{MHz}, \mathrm{CDCl}_{3}\right)$ and ${ }^{13} \mathrm{C}$ NMR of $5 \mathbf{g a}\left(125 \mathrm{MHz}, \mathrm{CDCl}_{3}\right)$

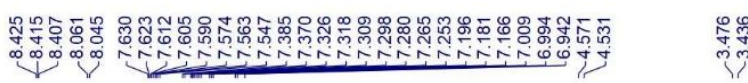
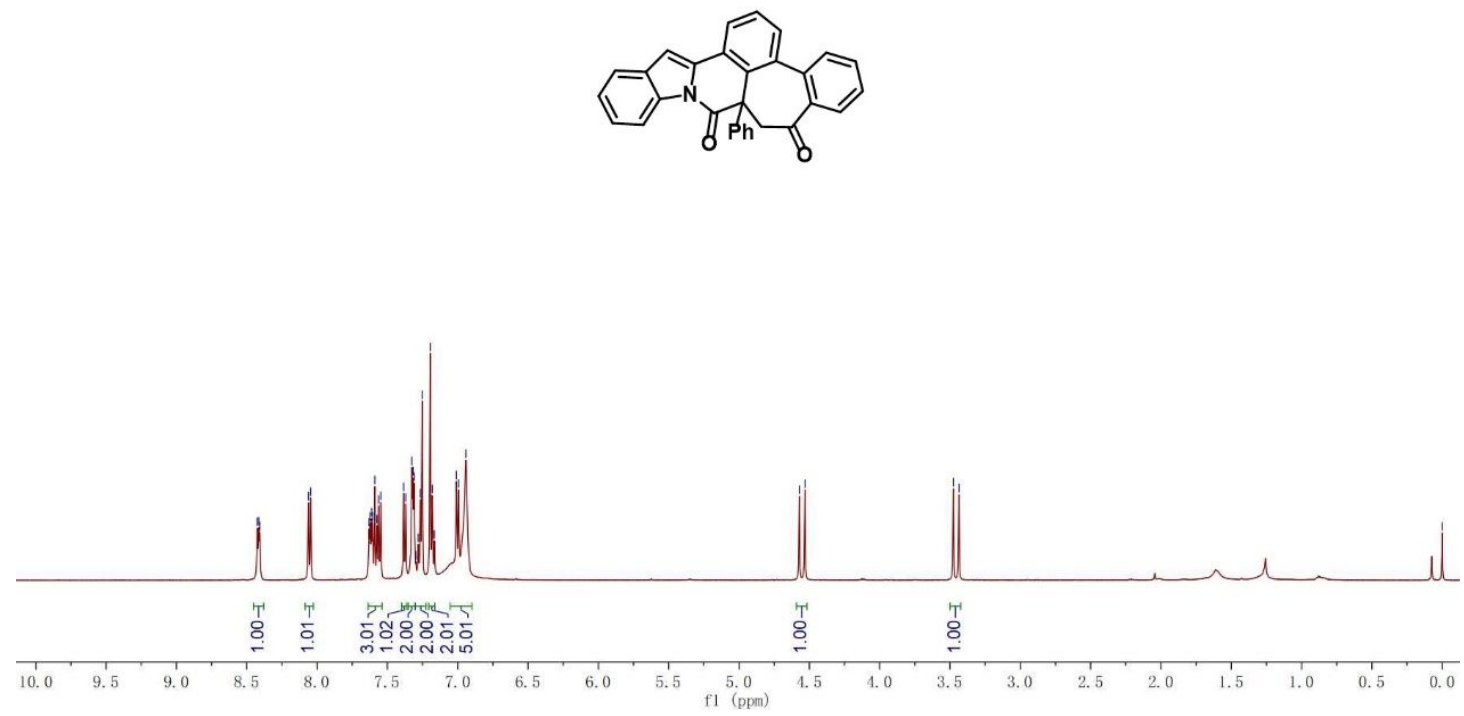

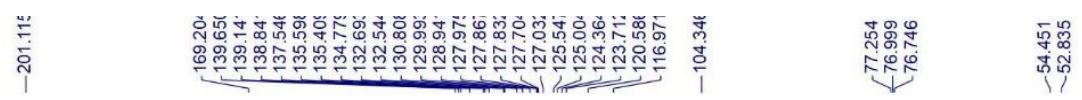


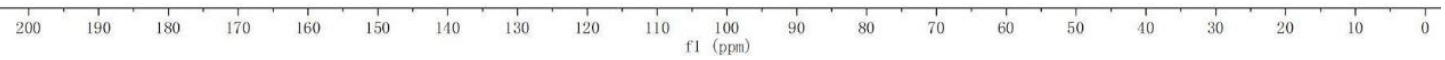


${ }^{1} \mathrm{H}$ NMR of $5 \mathbf{h a}\left(500 \mathrm{MHz}, \mathrm{CDCl}_{3}\right)$ and ${ }^{13} \mathrm{C}$ NMR of 5 ha $\left(125 \mathrm{MHz}, \mathrm{CDCl}_{3}\right)$




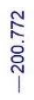
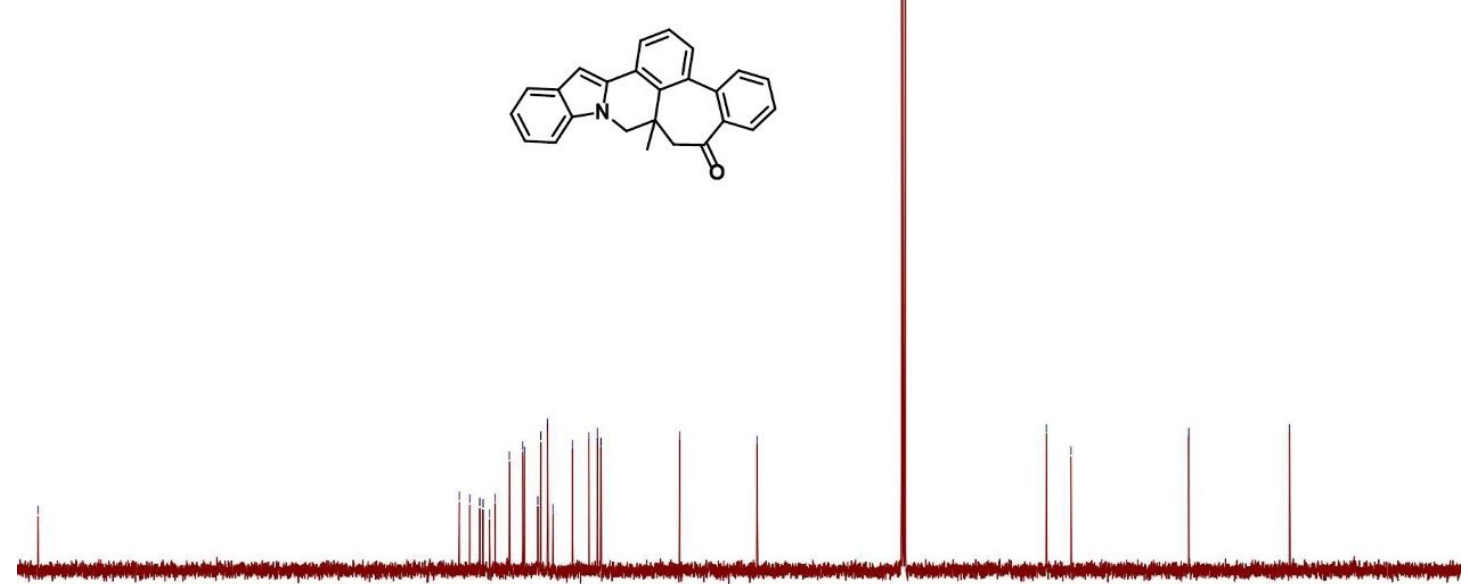
${ }^{1} \mathrm{H}$ NMR of $5 \mathbf{a l}\left(500 \mathrm{MHz}, \mathrm{CDCl}_{3}\right)$ and ${ }^{13} \mathrm{C}$ NMR of $5 \mathbf{a l}\left(125 \mathrm{MHz}, \mathrm{CDCl}_{3}\right)$





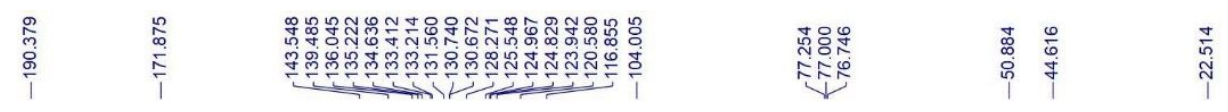
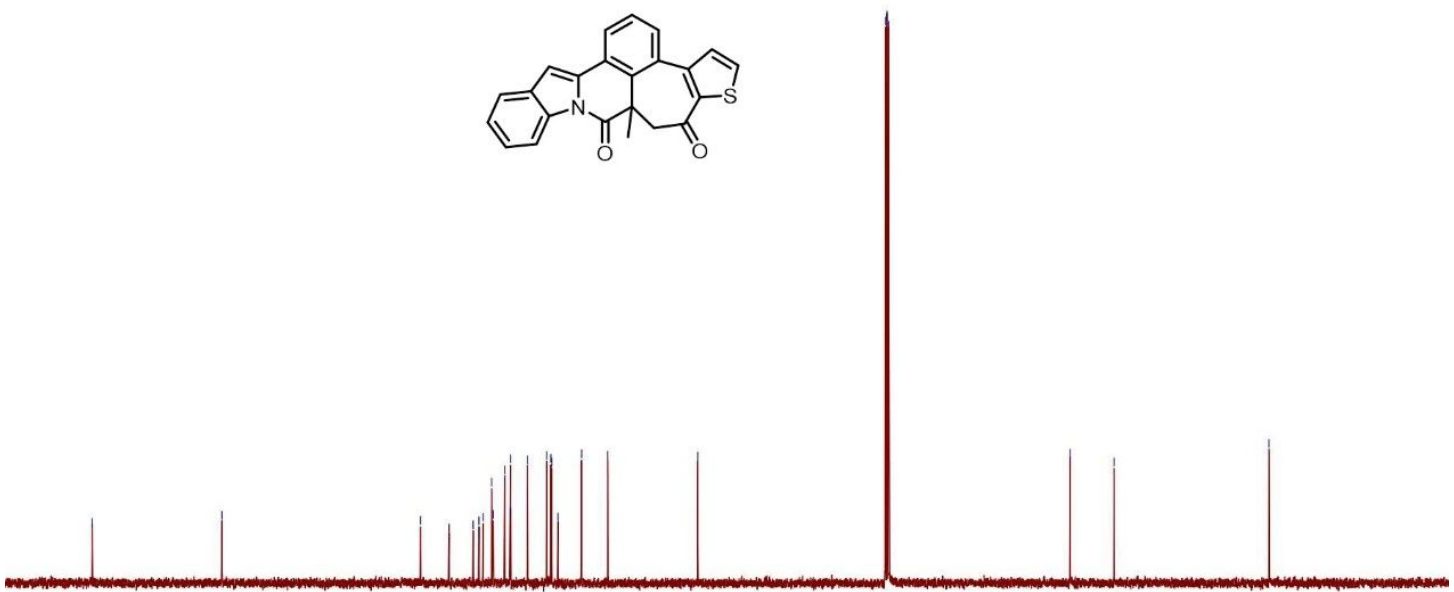

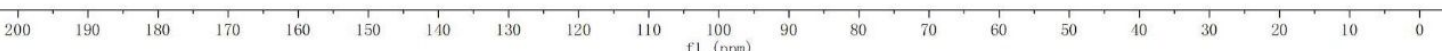




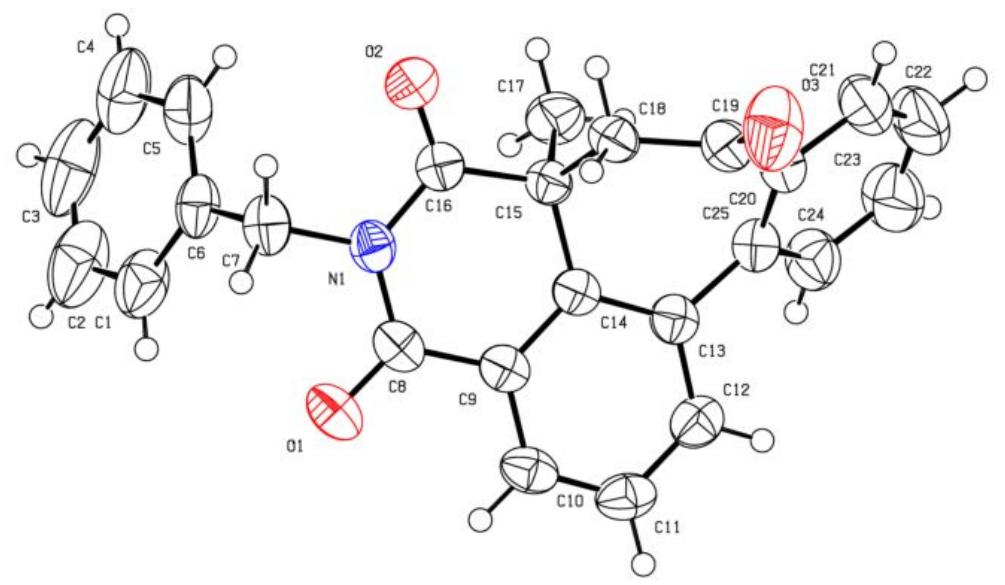

Figure S1 X-ray crystal structure of 3aa. The thermal ellipsoids are 50\% probability level.

Table S1 Crystal data and structure refinement for zlw-1.

Identification code

Empirical formula

Formula weight

Temperature/K

Wavelength

Crystal system, space group

$$
\begin{aligned}
& \mathrm{a} / \AA \\
& \mathrm{b} / \AA \\
& \mathrm{c} / \AA \\
& \alpha{ }^{\circ} \\
& \beta /{ }^{\circ} \\
& \gamma /{ }^{\circ}
\end{aligned}
$$

Volume $/ \AA^{3}$

$\mathrm{Z}$

$\rho \mathrm{g} / \mathrm{cm}^{3}$

$\mu / \mathrm{mm}^{-1}$

$\mathrm{F}(000)$

Crystal size $/ \mathrm{mm}^{3}$

Theta range for data collection

Limiting indices

Reflections collected / unique

Completeness to theta $=25.242$

Absorption correction

Refinement method

Data/restraints/parameters
$\mathrm{C}_{25} \mathrm{H}_{19} \mathrm{NO}_{3}$

381.41

296(2) K

$0.71073 \mathrm{~A}$

Monoclinic, P 21 /c

$9.5927(6)$

$8.6068(5)$

$23.6634(14)$

90

$93.0820(10)$

90

$1950.9(2)$

4

1.299

0.085

800

$0.300 \times 0.210 \times 0.200$

2.519 to $25.009 \mathrm{deg}$.

$-11<=\mathrm{h}<=11,-10<=\mathrm{k}<=10,-27<=\mathrm{l}<=28$

$19792 / 3445[\mathrm{R}($ int $)=0.0215]$

$97.5 \%$

None

Full-matrix least-squares on $\mathrm{F}^{\wedge} 2$

3445 / 0 / 262 
Goodness-of-fit on $\mathrm{F}^{2}$

Final $\mathrm{R}$ indices $[\mathrm{I}>2 \operatorname{sigma}(\mathrm{I})]$

$\mathrm{R}$ indices (all data)

Largest diff. peak/hole / e $\AA^{-3}$
1.102

$\mathrm{R} 1=0.0460, \mathrm{wR} 2=0.1203$

$\mathrm{R} 1=0.0553, \mathrm{wR} 2=0.1276$

0.176 and -0.259 e. $\mathrm{A}^{\wedge}-3$ 